$$
\begin{aligned}
& \text { Aplicações do teorema da } \\
& \text { transversalidade à genericidade } \\
& \text { em alguns } \\
& \text { problemas de contorno elípticos }
\end{aligned}
$$

Marcone Corrêa Pereira

\author{
DISSERTAÇÃO APRESENTADA \\ $\mathrm{AO}$ \\ INSTITUTO DE MATEMÁTICA E ESTATÍSTICA \\ DA \\ UNIVERSIDADE DE SÃO PAULO \\ PARA \\ OBTENÇÃO DO GRAU DE MESTRE \\ EM \\ MATEMÁTICA \\ Área de Concentração: Análise \\ Orientador: Prof. Antônio L. Pereira .
}

Durante a elaboração deste trabalho o autor recebeu apoio financeiro do $C N P q$

São Paulo, março de 2001 


\section{Aplicações do teorema da transversalidade à genericidade em alguns problemas de contorno elípticos}

Este exemplar corresponde à redação final da dissertação devidamente corrigida e defendida por Marcone Corrêa Pereira e aprovada pela comissão julgadora.

São Paulo, 28 de março de 2001.

Banca examinadora:

- Prof. Dr. Antônio L. Pereira (Presidente) - IME-USP

- Profa. Dra. Marina Pizzotti - IME-USP

- Prof. Dr. Orlando Lopes - IMECC - UNICAMP 
"Portanto todo aquele que ouve estas minhas palavras e as pratica, será semelhante ao homem prudente, que edificou a sua casa sobre a rocha. Desceu a chuva, transbordaram os rios, sopraram os ventos e deram contra aquela casa; contudo, ela não caiu porque estava edificada sobre a rocha." Mateus 7:24,25. 


\section{Agradecimentos}

A Deus por mais esta prova de amor.

Aos meus pais, Áurea e Manoel, que me deram todo apoio e amor.

À Raquel pela companhia, carinho, incentivo e amor.

À tia Gracelina e tio Antônio que cuidaram de mim durante todo este tempo em São Paulo juntamente com meus primos.

Ao meu orientador Professor Antônio Luiz Pereira pela amizade, paciência, apoio, incentivo, cuidado e disposição em ensinar.

À professora Marina pela cooperação no nosso trabalho.

Aos professores Luiz Augusto, Plínio, Rosa, Lúcia, Clodoaldo, Verderesi, Glads, Pedro Fagundes e Sérgio Oliva pelas oportunidades de discussão e aprendizado.

A Francisca, Feijão e Pinho pelo apoio eficiente.

Ao Antônio por me ensinar Latex.

Aos colegas Plácido, Marcelo, Marcelo Passos, Davi, Emivan, Albetan, Regina, Leonardo e Alexandre pela companhia e colaboração. 


\section{Resumo}

Nesta dissertação apresentamos cinco aplicações do teorema da Transversalidade enunciado e provado por D. Henry em [11]. Os quatro primeiros exemplos são de perturbação de fronteira para o problema de Dirichlet em equações elípticas. O último exemplo é uma aplicação do teorema da Transversalidade para uma equação parabólica unidimensional com condições de contorno de Neumann. 


\begin{abstract}
In this dissertation we present five applications of the Tranversality Theorem in a version due to D. Henry [11]. In the first four examples we consider perturbation of the boundary problems with Dirichlet boundary conditions. In the last example we apply the Transversality Theorem to obtain generic hyperbolicity for the equilibria of a unidimensional parabolic equation with Neumann boundary conditions.
\end{abstract}




\section{Índice}

Agradecimentos $\quad$ iv

Resumo $\quad$ v

Abstract vi vi

Introdução 1

1 Perturbação de Contorno 3

1.1 Definições, notações e resultados preliminares . . . . . . . . . . . . . 3

1.2 Perturbação de Contorno $\ldots \ldots \ldots \ldots \ldots \ldots \ldots$

1.3 Cálculo da diferencial $\ldots \ldots \ldots \ldots$. . . . . . . . . . . . . . . . 11

2 Operadores de Fredholm e o Teorema da Transversalidade 16

2.1 Operadores de Fredholm . . . . . . . . . . . . . . . . . . . . . . . . 16

2.2 O Teorema da Transversalidade . . . . . . . . . . . . . . . . . . . . . 24

3 Aplicações $\quad 27$

3.1 Preliminares. . . . . . . . . . . . . . . . . . . . . . . . . . 28

3.2 Simplicidade Genérica dos Autovalores do Laplaciano . . . . . . . . . . . 30

3.3 Uma Propriedade Genérica para as Autofunções do Laplaciano . . . . . . . 33

3.4 Simplicidade Genérica dos Autovalores Reais . . . . . . . . . . . . . . . . 40

3.5 Simplicidade Genérica das soluções de $\Delta u+f(x, u, \nabla u)=0 \ldots \ldots$. . . . 42

3.6 Hiperbolicidade Genérica dos Equilíbrios de $u_{t}=\left(a u_{x}\right)_{x}+f(u) \ldots \ldots . \quad 49$ 
Índice

Referências Bibliográficas 


\section{Introdução}

O problema de perturbação de fronteira recebeu atenção apenas esporádica na literatura matemática até o trabalho exaustivo de D. Henry em [11] (onde também se encontram muitas referências). Nessa obra o autor introduz novos métodos e obtem vários resultados inéditos, além de simplificar resultados conhecidos.

O objetivo central desta dissertação é expor alguns desses resultados e métodos. Em especial apresentamos uma versão generalizada do Teorema da Transversalidade de Abraham e Thom obtida em [11] com algumas aplicações.

No capítulo 1 algumas definições e notações são dadas bem como teoremas preliminares, alguns sem demonstração. Neste mesmo capítulo introduzimos a técnica desenvolvida por Henry em [11] para trabalhar com o tema de perturbação de fronteira.

No capítulo 2 desta dissertação enunciamos a versão generalizado do teorema da Transversalidade por Henry, definimos operadores de Fredholm no espaço dos operadores limitados e provamos algumas propriedades importantes destes operadores que são úteis nos exemplos apresentados no capítulo 3.

O terceiro e último capítulo é sem dúvida o mais importante pois é nele que algumas aplicações do teorema da Transversalidade são desenvolvidas. O primeiro exemplo é a prova de que os autovalores do Laplaciano com condições de contorno de Dirichlet são genericamente simples no conjunto das regiões limitadas $\mathcal{C}^{3}$-regulares do $\mathbb{R}^{n}$. Este resultado foi originalmente demonstrado por Micheletti [15] e Uhlenbeck [17] mas uma prova curta é apresentada aqui. Nosso segundo exemplo está intimamente relacionado com o primeiro e é a prova de que as autofunções $u$ do Laplaciano com condições de contorno de Dirichlet satisfazem genericamente no conjunto das regiões $\int_{\Omega} g(u) \neq 0$ onde $g$ é uma função real qualquer de classe $\mathcal{C}^{3}$. Este problema foi originalmente proposto por K. Rybakowski para $g(u)=u^{3}$ em conexão com um problema de bifurcação e foi generalizado sem maiores dificuldades. A prova de tal resultado é simples e exemplifica de maneira elegante a versão 
do teorema da Transversalidade apresentada aqui.

O terceiro exemplo do capítulo 3 é apenas uma generalização do primeiro exemplo. Consideramos agora um operador uniformemente elíptico geral em vez do Laplaciano e provamos que também nesse caso todos os autovalores reais do problema de Dirichlet são genericamente simples no conjunto das regiões limitadas $\mathcal{C}^{3}$-regulares. Este exemplo é aplicado no quarto exemplo que é a prova de que a equação não linear $\Delta u+f(\cdot, u, \nabla u)=0$ com condições de contorno de Dirichlet possui todas as soluções simples genericamente no conjunto das regiões limitadas $\mathcal{C}^{3}$-regulares. Este exemplo é mais trabalhoso e requer o uso da versão generalizada do teorema da Transversalidade.

O quinto e último exemplo é tambem uma aplicação do teorema da Transversalidade mas não é um exemplo de perturbação de fronteira. Provaremos que, para uma função monótona fixada $a \in \mathcal{C}^{2}([0,1],(0,+\infty))$, genericamente no conjunto das funções reais de classe $\mathcal{C}^{2}$ na topologia de Whitney todos os equilíbrios da equação

$$
u_{t}=\left(a u_{x}\right)_{x}+f(u), 0<x<1
$$

com condições de contorno de Neumann $u_{x}(t, 0)=u_{x}(t, 1)=0$ são hiperbólicos.

P. Brunovsky e S. Chow em [7] provaram tal afirmação para $a$ constante. A. L. Pereira em [16] prova que para uma função $f$ fixa todos os equilíbrios de (1) são genericamente hiperbólicos com relação a função $a$ e que para uma função $a$ fixada todos os equilíbrios de (1), satisfazendo uma hipótese adicional, são genericamente hiperbólicos em $f$ na topologia de Whitney.

O problema no caso geral permanece, tanto quanto saibamos, em aberto. 
Capítulo 1

\section{Perturbação de Contorno}

Para tratar problemas de variação de domínios em problemas de EDP D. Henry desenvolve em [11] uma espécie de cálculo diferencial onde a variável independente é o domínio.

Neste capítulo preliminar vamos introduzir a técnica desenvolvida em [11] para tratar este tipo de problema bem como notações e alguns outros resultados que serão necessários nas aplicações.

\subsection{Definições, notações e resultados preliminares}

Denotaremos um ponto $x$ qualquer do espaço $n$-dimensional Euclidiano $\mathbb{R}^{n}$ por $x=\left(x_{1}, \ldots, x_{n}\right)$.

$$
\left(\frac{\partial}{\partial x}\right)^{\alpha}={\frac{\partial}{\partial x_{1}}}^{\alpha_{1}} \ldots{\frac{\partial}{\partial x_{n}}}^{\alpha_{n}}
$$

onde $\alpha=\left(\alpha_{1}, \ldots, \alpha_{n}\right) \in \mathbb{N}^{n}$ e a $|\alpha|=\alpha_{1}+\ldots+\alpha_{n}$. De maneira análoga $x^{\alpha}=x_{1}^{\alpha_{1}} \ldots x_{n}^{\alpha_{n}}$. Usaremos também a seguinte notação para derivadas parciais,

$$
D_{i}=\frac{\partial}{\partial x_{i}} \text { e } D^{\alpha}=D_{1}^{\alpha_{1}} \ldots D_{n}^{\alpha_{n}}
$$

Seja $f$ uma aplicação que assume valores reais $m$-diferenciável em $x \in \mathbb{R}^{n}$. A $m$-ésima derivada de $f$ em $x$ pode ser considerada como uma $m$-forma linear simétrica sobre $\mathbb{R}^{n}$,

$$
h \longrightarrow D^{m} f(x) h^{m} .
$$

Então a norma $|\cdot|$ de $D^{m} f(x)$ pode ser definida como o

$$
\max _{|h| \leq 1}\left|D^{m} f(x) h^{m}\right| .
$$

Vale a pena observar que a $m$-ésima derivada de $f$ em $x$ pode ser considerada também como uma coleção de derivadas parciais de $f$, ou seja,

$$
D^{m} f(x)=\left\{\left(\frac{\partial}{\partial x}\right)^{\alpha} ;|\alpha|=m\right\} .
$$


Denotaremos por $\Omega$ um subconjunto aberto do $\mathbb{R}^{n}$ com fronteira $\partial \Omega$ e fecho $\bar{\Omega}$. Se $E$ é algum espaço vetorial normado, $\mathcal{C}^{m}(\Omega, E)$ é o espaço das funções limitadas $m$-diferenciáveis $f$ sobre $\Omega$ com derivadas limitadas e valores em $E$ cujas derivadas se extendem continuamente até $\bar{\Omega}$ com a norma usual

$$
\|f\|_{C^{m}(\Omega, E)}=\max _{0 \leq j \leq m} \sup _{x \in \Omega}\left|D^{m} f(x)\right| .
$$

Se o espaço vetorial normado $E=\mathbb{R}$, escreveremos apenas $\mathcal{C}^{m}(\Omega)$.

$\mathcal{C}_{0}^{m}(\Omega)$ é o subeconjunto de $\mathcal{C}^{m}(\Omega)$ consistindo de todas as funções com suporte compacto em $\Omega$.

$\mathcal{C}_{\text {unif }}^{m}(\Omega, E)$ é o subespaço fechado de $\mathcal{C}^{m}(\Omega, E)$ consistindo das funções cuja a $m$-ésima derivada é uniformemente contínua. Observe que se $\Omega$ é limitado, este conjunto é o próprio $\mathcal{C}^{m}(\Omega, E)$.

$\mathcal{C}^{m, \alpha}(\Omega, E)$ é o espaço consistindo de todas as funções cuja a $m$-ésima derivada é Holder contínua com expoente $\alpha, 0<\alpha \leq 1$, e norma

$$
\|f\|_{\mathcal{C}^{m, \alpha}(\Omega, E)}=\max \left\{\|f\|_{\mathcal{C}^{m}(\Omega, E)}, H_{\alpha}^{\Omega}\left(D^{m} f\right)\right\}
$$

onde

$$
H_{\alpha}^{\Omega}(f)=\sup \left\{\frac{|f(x)-f(y)|}{|x-y|^{\alpha}} ; x \neq y \in \Omega\right\}
$$

Dizemos que um conjunto aberto $\Omega \subset \mathbb{R}^{n}$ é $\mathcal{C}^{m}$-regular se existe $\phi \in \mathcal{C}^{m}\left(\mathbb{R}^{n}, \mathbb{R}\right)$, que é pelo menos $\mathcal{C}_{\text {unif }}^{1}\left(\mathbb{R}^{n}, \mathbb{R}\right)$, tal que

$$
\Omega=\left\{x \in \mathbb{R}^{n} ; \phi(x)>0\right\}
$$

e $\phi(x)=0$ implica $|\nabla \phi| \geq 1$.

D. Henry em [11] mostra, para abertos limitados, que esta definição de região $\mathcal{C}^{m}$-regular é equivalente às definições usadas por E. Browder [4] e Agmon-Douglis-Nirenberg [6] em seus artigos de problemas de contorno para operadores elípticos.

Seja $m$ um inteiro não negativo, $p$ um número real $\geq 1$ e $\Omega$ um aberto limitado. Para uma função $u \in \mathcal{C}^{m}(\Omega)$ defina a norma

$$
\|u\|=\left(\int_{\Omega} \sum_{|\alpha| \leq m}\left|D^{\alpha} u\right|^{p} d x\right)^{\frac{1}{p}} .
$$

O espaço vetorial $\mathcal{C}^{m}(\Omega)$ não é completo sob a norma (1.1). Completando tal espaço, obtemos um espaço de Banach que denotamos por $H^{m, p}(\Omega)$. Fixamos a notação $\|\cdot\|_{H^{m, p}(\Omega)}$ para a norma (1.1) em $H^{m, p}(\Omega)$. 
Introduzimos outro espaço linear $W^{m, p}(\Omega)$ da seguinte maneira. Uma função de valores reais $u$ pertence a $W^{m, p}(\Omega)$ se $u \in L^{p}(\Omega)$ e se todas as suas derivadas fracas de ordem menor ou igual a $m$ pertencem a $L^{p}(\Omega)$. Friedman prova em [9] que se $\partial \Omega$ é $\mathcal{C}^{m}$-regular então $W^{m, p}(\Omega)=H^{m, p}(\Omega)$. Quando $p=2$ tem-se a notação especial $H^{m, p}(\Omega)=H^{m}(\Omega)$.

De maneira análoga introduzimos os espaços $H_{0}^{m, p}(\Omega)$ e $W_{0}^{m, p}(\Omega)$, onde $H_{0}^{m, p}(\Omega)$ é o completamento do espaço $\mathcal{C}_{0}^{m}(\Omega)$ e $W_{0}^{m, p}(\Omega)$ é o espaço das funções $u$ cuja $m$-ésima derivada pertence a $L^{p}(\Omega)$ satisfazendo $D^{\alpha} u=0$ para todo $|\alpha| \leq m$ em $\partial \Omega$.

Teorema 1.1 Seja $\Omega \subset \mathbb{R}^{n}$ uma região $\mathcal{C}^{2}$-regular e $h \in \mathcal{C}^{2}\left((-\epsilon, \epsilon) \times \Omega, \mathbb{R}^{n}\right)$ para algum $\epsilon>0 \operatorname{com} h(0, \cdot)=i_{\Omega}$. Seja também $f: \mathbb{R} \times \mathbb{R}^{n} \longrightarrow \mathbb{R} \mathcal{C}^{2}$ pelo menos numa vizinhança de $t=0$ com suporte compacto. Então, se $\Omega(t)=h(t, \Omega), t \longrightarrow \int_{\Omega} f(t, x) d x$ é $\mathcal{C}^{1}$ próximo de $t=0 e$

$$
\frac{d}{d t} \int_{\Omega(t)} f(t, x) d x=\int_{\Omega(t)} \frac{\partial f}{\partial t}(t, x) d x+\int_{\partial \Omega(t)} f(t, x) V \cdot N d A_{x}
$$

onde $V$ é o campo de vetores $\frac{\partial h}{\partial t}(t, x)=V(t, h(t, x))$ e $N$ é o vetor normal unitário apontando para fora de $\Omega$.

Prova. É suficiente calcular tal derivada em $t=0$.

$$
h(t, x)=x+t V(x)+O\left(t^{2}\right)
$$

que nos dá

$$
\begin{aligned}
\frac{\partial h}{\partial x}(t, x)=I+t V_{x}(x)+O\left(t^{2}\right) \\
\int_{\Omega(t)} f(t, y) d y=\int_{\Omega} f(t, h(t, x)) \operatorname{det}\left(\frac{\partial h}{\partial x}(t, x)\right) d x \\
=\int_{\Omega}\left\{f(0, x)+t\left(f_{t}(0, x)+f_{x}(0, x) \cdot V+f(0, x) \operatorname{det}^{\prime}(I) \cdot V_{x}(x)\right)+O\left(t^{2}\right)\right\} d x \\
=\int_{\Omega}\left\{f(0, x)+t\left(f_{t}(0, x)+f_{x}(0, x) \cdot V+f(0, x) \operatorname{traco} V_{x}(x)\right)+O\left(t^{2}\right)\right\} d x \\
=\int_{\Omega}\left\{f(0, x)+t\left(f_{t}(0, x)+f_{x}(0, x) \cdot V+f(0, x) \operatorname{div} V(x)\right)+O\left(t^{2}\right)\right\} d x .
\end{aligned}
$$

Portanto em $t=0$ temos que

$$
\begin{aligned}
\frac{d}{d t} \int_{\Omega} f(0, y) d y & =\int_{\Omega}\left\{f_{t}(0, x)+f_{x}(0, x) \cdot V+f(0, x) d i v V(x)\right\} d x \\
& =\int_{\Omega}\left\{f_{t}(0, x)+\operatorname{div}(f(0, x) V(x))\right\} d x \\
& =\int_{\Omega} f_{t}(0, x) d x+\int_{\partial \Omega} f(0, x) V(x) \cdot N d A_{x}
\end{aligned}
$$


de onde segue o resultado desejado.

Algumas vezes se faz necessário o uso de operadores diferenciais em hipersuperfícies $S \subset \mathbb{R}^{n}$. As seguintes definições são intrínsecas a $S$ e nada dizem sobre o ambiente $\mathbb{R}^{n}$.

Seja $S$ uma hipersuperfície $\mathcal{C}^{1}$ em $\mathbb{R}^{n}$ e seja $\phi: S \longrightarrow \mathbb{R}$ de classe $\mathcal{C}^{1}$ de maneira que possa ser estendida $\mathcal{C}^{1}$ a uma vizinhança $S$. Então $\nabla_{S} \phi$ é o campo vetorial tangente sobre $S$ tal que para cada curva $x(t) \subset S$ de classe $\mathcal{C}^{1}$, temos

$$
\frac{d}{d t} \phi(x(t))=\nabla_{S} \phi(x(t)) \cdot \dot{x}(t)
$$

Seja $S$ uma hipersuperfície $\mathcal{C}^{2}$ em $\mathbb{R}^{n}$ e $\vec{a}: S \longrightarrow \mathbb{R}^{n}$ um campo vetorial $\mathcal{C}^{1}$, definimos $\operatorname{div}_{S} \vec{a}: S \longrightarrow \mathbb{R}^{n}$ como a função contínua tal que, para toda função $\phi: S \longrightarrow \mathbb{R}$ de classe $\mathcal{C}^{1}$ com suporte compacto em $S$,

$$
\int_{S}\left(\operatorname{div}_{S} \vec{a}\right) \phi=-\int_{S} \vec{a} \cdot \nabla_{S} \phi
$$

Finalmente, se $u: S \longrightarrow \mathbb{R}$ é $\mathcal{C}^{2}$, então $\Delta_{S} u=\operatorname{div}_{S}\left(\nabla_{S} u\right)$ ou equivalentemente, para toda $\phi: S \longrightarrow \mathbb{R} \mathcal{C}^{1}$ com suporte compacto em $S$,

$$
\int_{S} \phi \Delta_{S} u=-\int_{S} \nabla_{S} \phi \cdot \nabla_{S} u
$$

Teorema 1.2 1. Se $S$ é uma hipersuperfície $\mathcal{C}^{1}$ e $\phi: \mathbb{R}^{n} \longrightarrow \mathbb{R}$ é $\mathcal{C}^{1}$ numa vizinhança de $S$, entâo, sobre $S, \nabla_{S} \phi(x)$ é a componente do $\nabla \phi(x)$ tangente a $S$ em $x$, ou seja,

$$
\nabla_{S} \phi(x)=\nabla \phi(x)-\frac{\partial \phi}{\partial N}(x) N
$$

onde $N$ é um campo vetorial normal sobre $S$.

2. Se $S$ é uma hipersuperfície $\mathcal{C}^{2}$ em $\mathbb{R}^{n} \vec{a}: S \longrightarrow \mathbb{R}^{n}$ é um campo vetorial $\mathcal{C}^{1}$ numa vizinhança de $S, N: \mathbb{R}^{n} \longrightarrow \mathbb{R}^{n}$ é um campo vetorial unitário sobre uma vizinhança de $S$, e $N$ sobre $S$ é o campo normal a $S$ nos pontos de $S$. Então

$$
\operatorname{div}_{S} \vec{a}=\operatorname{div} \vec{a}-(\operatorname{div} N) \vec{a} \cdot N-\frac{\partial}{\partial N}(a \cdot N)
$$

sobre $S$.

3. Se $S$ é uma hipersuperfície $\mathcal{C}^{2}, u: \mathbb{R}^{n} \longrightarrow \mathbb{R}$ é $\mathcal{C}^{2}$ sobre uma vizinhança de $S$ e $N$ é um campo vetorial normal unitário a $S$ no sentido de 2 acima. Então

$$
\Delta_{S} u=\Delta u-\operatorname{div} N \frac{\partial u}{\partial N}-\frac{\partial^{2} u}{\partial N^{2}}+\nabla_{S} u \cdot \frac{\partial N}{\partial N}
$$

sobre $S$. 
Prova. Veja [10].

Frequentemente citaremos o teorema de unicidade de Cauchy para equações elípticas de segunda ordem. Enunciaremos este resultado com detalhes aqui. A prova pode ser encontrada em Hormander [13] teorema 8.9.1, ou em Agmon [3] assumindo $a_{i j}$ de classe $\mathcal{C}^{2}$

Teorema 1.3 Suponha que $Q \subset \mathbb{R}^{n}$ é um aberto conexo; $B$ é uma bola que intercepta $\partial Q$ numa hipersuperfície $B \cap \partial Q ; a_{i j}=a_{j i}: Q \longrightarrow \mathbb{R}$ é uma função $\mathcal{C}^{1}$ com $\sum_{i, j=1}^{n} a_{i j}(x) \xi_{i} \xi_{j} \geq$ $c_{0}|\xi|^{2} \forall x \in Q$ e $\xi \in \mathbb{R}^{n}$ para alguma constante $c_{0}>0$. Assuma que $u \in H^{2}(Q)$ e que para alguma constante $K$

$$
\left|\sum_{i, j=1}^{n} a_{i j}(x) \frac{\partial^{2} u}{\partial x_{i} x_{j}}\right| \leq K(|\nabla u(x)|+|u(x)|)
$$

para quase todo ponto de $Q$ e que em quase todo ponto de $B \cap \partial Q$ temos $u=0$ e $\frac{\partial u}{\partial N}=0$. Então $u=0$ em quase todo ponto de $Q$.

\subsection{Perturbação de Contorno}

Consideremos o operador linear diferencial matricial formal

$$
L u(x)=\left(u(x), \frac{\partial u}{\partial x_{1}}(x), \ldots, \frac{\partial u}{\partial x_{n}}(x), \frac{\partial^{2} u}{\partial x_{1}^{2}}(x), \frac{\partial^{2} u}{\partial x_{1} \partial x_{2}}(x), \ldots\right), x \in \mathbb{R}^{n}
$$

com a quantidade de termos que se fizer necessário. Dada uma função $f$ de várias variáveis podemos definir um operador diferencial formal não linear por

$$
v(x)=f(x, L u(x)), x \in \mathbb{R}^{n} .
$$

Mais precisamente, suponha que $L u(\cdot)$ assume valores em $\mathbb{R}^{p}$ e $f(x, \lambda)$ está definida para $(x, \lambda)$ em algum conjunto aberto $O \subset \mathbb{R}^{n} \times \mathbb{R}^{p}$. Para subconjuntos $\Omega \subset \mathbb{R}^{n}$ definimos $F_{\Omega}$ por

$$
F_{\Omega}(u)(x)=f(x, L u(x)), x \in \Omega
$$

para funções $u$ suficientemente suaves sobre $\Omega$ tal que $(x, L u(x)) \in O$ para todo $x \in \bar{\Omega}$. Por exemplo, se $f$ é contínua, $\Omega$ é limitado e $L$ envolve derivadas de ordem menor ou igual a $m$, o domínio de $F_{\Omega}$ é um subconjunto aberto de $\mathcal{C}^{m}(\Omega)$, (talvez vazio) e os valores de $F_{\Omega}$ se encontram em $\mathcal{C}^{0}(\Omega)$. Outros espaços de funções podem ser usados com modificações naturais. 
Seja $h: \Omega \longrightarrow \mathbb{R}^{n}$ um mergulho $m$ vezes diferenciável, isto é, um difeomorfismo sobre sua imagem $h(\Omega)$ de ordem $m$. Definimos a aplicação de composição (ou o pull-back) $h^{*}$ de $h$ por

$$
h^{*} u(x)=(u \circ h)(x)=u(h(x)), x \in \Omega
$$

onde $u$ é uma função definida em $h(\Omega)$.

Proposição $1.4 h^{*}: \mathcal{C}^{m}(h(\Omega)) \longrightarrow \mathcal{C}^{m}(\Omega): u \longrightarrow u \circ h$ é um isomorfismo com inversa $h^{*-1}=\left(h^{-1}\right)^{*}$.

Prova. Seja $h: \Omega \longrightarrow h(\Omega)$ um mergulho de ordem $m, h^{*} u \in \mathcal{C}^{m}(\Omega)$ para todo $u \in$ $\mathcal{C}^{m}(h(\Omega))$ pela regra da cadeia, portanto $h^{*}$ está bem definido. Observe também que $h^{*}$ é limitado, de fato, $\left\|h^{*} u\right\|_{\mathcal{C}^{m}(\Omega)}=\|u \circ h\|_{\mathcal{C}^{m}(\Omega)} \leq c\|u\|_{\mathcal{C}^{m}(h(\Omega))}$ para algum $c>0$ já que $h \in \mathcal{C}^{m}\left(\Omega, \mathbb{R}^{n}\right)$. Além disso $h^{*}$ é claramente linear, injetora e sobrejetora. Logo $h^{*}$ é um isomorfismo já que $h^{*-1}=\left(h^{-1}\right)^{*}$ também satisfaz a desigualdadeque acima.

Proposição 1.5 Sejam $h_{1}: \Omega_{0} \longrightarrow h_{1}\left(\Omega_{0}\right)=\Omega_{1}$ e $h_{2}: \Omega_{1} \longrightarrow h_{2}\left(\Omega_{1}\right)=\Omega_{2}$ difeomorfismos sobre suas imagens onde $\Omega_{i} \subset \mathbb{R}^{n}$ para $i=0,1,2$. Então $\left(h_{2} \circ h_{1}\right)^{*}=h_{1}^{*} h_{2}^{*}$.

Prova. Temos que

$$
\left(h_{2} \circ h_{1}\right)^{*} v=v \circ h_{2} \circ h_{1}=h_{1}^{*}\left(v \circ h_{2}\right)=h_{1}^{*}\left(h_{2}^{*} v\right)=h_{1}^{*} h_{2}^{*} v
$$

de onde segue o resultado.

É importante observar que as proposições anteriores são válidas em outros espaços de funções, como por exemplo, os espaços de Sobolev, $h^{*}: W^{k, p}(h(\Omega)) \longrightarrow W^{k, p}(\Omega) 0 \leq k \leq$ $m, 1 \leq p \leq \infty$

Dado um mergulho $h$ de uma região limitada $\Omega$ podemos considerar o operador diferencial $F_{h(\Omega)}$ com domínio aberto $D_{F_{h(\Omega)}} \subset \mathcal{C}^{m}(h(\Omega))$. A aplicação

$$
F_{h(\Omega)}: D_{F_{h(\Omega)}} \subset \mathcal{C}^{m}(h(\Omega)) \longrightarrow \mathcal{C}^{0}(h(\Omega))
$$

é chamada a forma Euleriana do operador diferencial formal não linear $v \longrightarrow f(\cdot, L v(\cdot))$ sobre $h(\Omega)$, enquanto

$$
h^{*} F_{h(\Omega)} h^{*-1}: h^{*} D_{F_{h(\Omega)}} \subset \mathcal{C}^{m}(\Omega) \longrightarrow \mathcal{C}^{0}(\Omega)
$$

é chamada forma Lagrangeana do mesmo operador diferencial formal não linear. Os mesmos termos são usados para outros espaços de funções. 
A forma Euleriana é frequentemente mais natural e simples para se fazer cálculos, a forma Lagrangeana é usada para provar teoremas. De fato, provaremos no capítulo de aplicações que, para nossas aplicações, ambos os métodos são equivalentes.

A vantagem da forma Lagrangeana é que ela age em espaços de funções que não dependem de $h$ facilitando então o uso de teoremas como o teorema da função implícita e o teorema da Transversalidade. Entretanto precisamos garantir que

$$
(u, h) \longrightarrow h^{*} F_{h(\Omega)} h^{*-1} u
$$

é diferenciável e calcular derivadas com respeito a $h$. Como $h^{*}$ é linear, a diferenciabilidade em relação a $u$ não apresenta problema algum. Antes de estudarmos a diferenciabilidade da aplicação (1.2), provaremos o seguinte resultado.

\section{Proposição 1.6}

$$
\operatorname{Diff}^{m}(\Omega)=\left\{h \in \mathcal{C}^{m}\left(\Omega, \mathbb{R}^{n}\right) ; h \text { é injetora } e \frac{1}{\left|\operatorname{deth}^{\prime}(x)\right|} \text { é limitado em } \Omega\right\}
$$

é aberto se $\Omega \subset \mathbb{R}^{n}$ é aberto limitado de classe $\mathcal{C}^{m}$.

Prova. Tome $h_{0} \in \operatorname{Diff}^{m}(\Omega)$ e suponha por absurdo que existe $\left\{h_{n}\right\} \subset \mathcal{C}^{m}\left(\Omega, \mathbb{R}^{n}\right)-\operatorname{Diff}^{m}(\Omega)$ tal que $\left\|h_{n}-h_{0}\right\|_{\mathcal{C}^{m}} \longrightarrow 0$ quando $n \longrightarrow \infty$. Então $\left\|h_{n}^{\prime}-h_{0}^{\prime}\right\|_{\mathcal{C}^{m-1}} \longrightarrow 0$ e como por hipótese $\frac{1}{\left|\operatorname{det} h_{0}^{\prime}(x)\right|}$ é limitado, existe $n_{0} \in \mathbb{N}$ tal que $\frac{1}{\left|\operatorname{deth}_{n}^{\prime}(x)\right|}$ é limitado para $n \geq n_{0}$. Se $h_{n} \notin \operatorname{Diff}^{m}(\Omega), h_{n}$ deve ser não injetora. Assim para todo $n \geq n_{0}$ existe $p_{n}, q_{n}$ tal que $h_{n}\left(p_{n}\right)=h_{n}\left(q_{n}\right)$. Sendo $\Omega$ limitado em $\mathbb{R}^{n}$ existem subseqüências $\left\{p_{n_{j_{k}}}\right\} \subset\left\{p_{n}\right\}$ e $\left\{q_{n_{j_{k}}}\right\} \subset\left\{q_{n}\right\}$ convergentes para $p$ e $q \in \bar{\Omega}$ tal que $h_{n_{j_{k}}}\left(p_{n_{j_{k}}}\right)=h_{n_{j_{k}}}\left(q_{n_{j_{k}}}\right)$. Suponha, sem perda de generalidade $p_{n} \longrightarrow p, q_{n} \longrightarrow q$ e $h_{n}\left(p_{n}\right)=h_{n}\left(q_{n}\right)$. Agora

$$
\begin{aligned}
\left|h_{0}(p)-h_{0}(q)\right| \leq & \left|h_{0}(p)-h_{0}\left(p_{n}\right)\right|+\left|h_{0}\left(p_{n}\right)-h_{n}\left(p_{n}\right)\right|+\left|h_{n}\left(p_{n}\right)-h_{n}\left(q_{n}\right)\right|+ \\
& +\left|h_{n}\left(q_{n}\right)-h_{0}\left(q_{n}\right)\right|+\left|h_{0}\left(q_{n}\right)-h_{0}(q)\right| \longrightarrow 0
\end{aligned}
$$

quando $n \longrightarrow \infty$. Então $p=q$ pois $h_{0}$ é injetora.

Portanto até agora nossa hipótese de absurdo resultou em duas seqüências $\left\{p_{n}\right\}$ e $\left\{q_{n}\right\}$ distintas convergindo para um mesmo ponto $p \in \bar{\Omega}$ tal que $h_{n}\left(p_{n}\right)=h_{n}\left(q_{n}\right)$. Mostraremos agora que existe uma vizinhança $U$ de $p$ em $\bar{\Omega}$ tal que todo $h$ suficientemente próximo de $h_{0}$ em $\mathcal{C}^{m}$ é injetora em $U$ implicando então que a partir de algum $n_{1}>n_{0}, h_{n}$ é injetora em $U$ e portanto $p_{n}=q_{n}$, contradição.

Seja $\theta=\inf \left\{\left\|h^{\prime}(p) \cdot k\right\| ;\|k\|=1\right.$ e $\left.\left\|h-h_{0}\right\|<\epsilon \operatorname{em} \mathcal{C}^{m}\right\}$.

(a) Afirmamos que $\theta>0$ se $\epsilon$ é suficientemente pequeno, de fato,

$$
\begin{aligned}
\left\|h^{\prime}(p) \cdot k\right\| & \geq\left\|h_{0}^{\prime}(p) \cdot k\right\|-\left\|\left(h^{\prime}(p)-h_{0}^{\prime}(p)\right) \cdot k\right\| \\
& \geq\left\|h_{0}^{\prime}(p) \cdot k\right\|-\left\|h^{\prime}(p)-h_{0}^{\prime}(p)\right\| .
\end{aligned}
$$


Basta tomar $2 \epsilon=i n f_{\|k\|=1}\left\|h_{0}^{\prime}(p) \cdot k\right\|$ (que é positivo pois $h_{0}, \in \operatorname{Diff}^{m}(\Omega)$ ) e $h$ suficientemente próximo a $h_{0}$ para que $\left\|h^{\prime}(p)-h_{0}^{\prime}(p)\right\| \leq \epsilon$.

(b) Afirmamos também que para $h$ numa vizinhança de $h_{0}$ e $p+k$ numa vizinhança de $p$ temos $\left\|h^{\prime}(p+k)-h^{\prime}(p)\right\| \leq \frac{\theta}{2}$. De fato,

$$
\begin{aligned}
\left\|h^{\prime}(p+k)-h^{\prime}(p)\right\| & \leq\left\|h^{\prime}(p+k)-h_{0}^{\prime}(p+k)\right\|+\left\|h_{0}^{\prime}(p+k)-h_{0}^{\prime}(p)\right\|+\left\|h_{0}^{\prime}(p)-h^{\prime}(p)\right\| \\
& <\frac{\theta}{6}+\frac{\theta}{6}+\frac{\theta}{6}<\frac{\theta}{2}
\end{aligned}
$$

se fizermos $\left\|h^{\prime}-h_{0}^{\prime}\right\|$ e $\left\|h_{0}^{\prime}(p+k)-h_{0}^{\prime}(p)\right\|<\frac{\theta}{6}$.

Tomando $V$ vizinhança de $h_{0}$ e $U$ vizinhança de $p$ para que (a) e (b) sejam válidos, temos

$$
h(p+k)=h(p)+\int_{0}^{1} h^{\prime}(p+t k) \cdot k d t \forall k \in U \text { e } \forall h \in V .
$$

Então podemos obter que

$$
\begin{aligned}
\|h(p+k)-h(p)\| & \geq\left\|\int_{0}^{1} h^{\prime}(p) \cdot k d t\right\|-\int_{0}^{1}\left\|h^{\prime}(p+t k)-h^{\prime}(p)\right\|\|k\| d t \\
& \geq\left\|h^{\prime}(p) \cdot k\right\|-\left\|h^{\prime}(p+\tau k)-h^{\prime}(p)\right\|\|k\| \\
& \geq\left\|h^{\prime}(p) \cdot \frac{k}{\|k\|}\right\|\|k\|-\left\|h^{\prime}(p+\tau k)-h^{\prime}(p)\right\|\|k\| \\
& \geq \frac{\theta}{2}\|k\|>0
\end{aligned}
$$

de onde segue o resultado.

Diferenciabilidade. Voltando a questão de suavidade, sejam $h \in \operatorname{Diff}^{m}(\Omega), v=$ $h^{*-1} u, y=h(x), x \in \Omega$

$$
h^{*} F_{h(\Omega)} h^{*-1} u(x)=F_{h(\Omega)} v(y)=f(y, L v(y))=f\left(h(x), h^{*} L h^{*-1} u(x)\right) .
$$

Consideremos primeiro o operador diferencial

$$
\left(\frac{\partial}{\partial y}\right)^{\alpha}=\prod_{i=1}^{n}\left(\frac{\partial}{\partial y_{i}}\right)^{\alpha_{i}}
$$

onde $\alpha=\left(\alpha_{1}, \ldots, \alpha_{n}\right)$ para $\alpha_{i}$ inteiro não-negativo. Pela regra da cadeia temos que

$$
\begin{aligned}
h^{*} \frac{\partial}{\partial y_{i}} h^{*-1} u(x) & =\frac{\partial}{\partial y_{i}}\left(u \circ h^{-1}\right)(h(x)) \\
& =\sum_{k=1}^{n} \frac{\partial u}{\partial x_{k}}\left(h^{-1}(y)\right) \frac{\partial\left(h^{-1}\right)_{k}}{\partial y_{i}}(h(x)) \\
& =\sum_{k=1}^{n} \frac{\partial u}{\partial x_{k}}\left(h^{-1}(y)\right)\left(h_{y}^{-1}\right)_{k i}(h(x)) \\
& =\sum_{k=1}^{n} \frac{\partial u}{\partial x_{k}}(x)\left(h_{x}^{-1}\right)_{k i}(x)
\end{aligned}
$$


o que nos dá

$$
h^{*}\left(\frac{\partial}{\partial y}\right)^{\alpha} h^{*-1} u(x)=\left(\frac{\partial}{\partial y}\right)^{\alpha} v(y)=\prod_{i=1}^{n}\left(\sum_{k=1}^{n}\left(h_{x}^{-1}\right)_{k i}(x) \frac{\partial}{\partial x_{k}}\right)^{\alpha_{i}} u(x) .
$$

Então, a aplicação

$$
F: \mathcal{C}^{m}(\Omega) \times \operatorname{Diff}^{m}(\Omega) \longrightarrow \mathcal{C}^{0}(\Omega):(u, h) \longrightarrow h^{*} L h^{*-1} u
$$

é função racional dos coeficientes de $h_{x}^{-1}$ e suas derivadas parciais de ordem menor ou igual a $m$, logo é analítica no aberto $\operatorname{Diff}^{m}(\Omega)$ em $h$. Portanto se $f$ é uma função $\mathcal{C}^{r}$ ou analítica em $O$ temos que a aplicação

$$
F: \mathcal{C}^{m}(\Omega) \times \operatorname{Diff}^{m}(\Omega) \longrightarrow \mathcal{C}^{0}(\Omega):(u, h) \longrightarrow h^{*} F_{h(\Omega)} h^{*-1} u=f\left(h(\cdot), h^{*} L h^{*-1} u\right)
$$

é $\mathcal{C}^{r}$ ou analítica respectivamente em seu domínio de definição. Outros espaços de funções podem ser empregados e os resultados de suavidade são essencialmente os mesmos.

\subsection{Cálculo da diferencial}

Em vista dos resultados da seção 1.2, para calcular a derivada de

$$
h \longrightarrow h^{*} F_{h(\Omega)} h^{*-1} u,
$$

basta calcular a derivada de Gâteaux, isto é, a t-derivada ao longo de uma curva de mergulhos $t \longrightarrow h(t, \cdot)$ de classe $\mathcal{C}^{1}$. Suponha então que queremos calcular

$$
\frac{\partial}{\partial t} F_{\Omega(t)}(v)(y)=\frac{\partial}{\partial t} f(y, L v(y))
$$

com $y=h(t, x)$ fixo em $\Omega(t)=h(t, \Omega)$. Para que $y$ permaneça fixo devemos ter $x=x(t)$, $y=h(t, x(t))$ tal que

$$
0=\frac{\partial h}{\partial t}+\frac{\partial h}{\partial x} x^{\prime}(t) \Longrightarrow x^{\prime}(t)=-\left(\frac{\partial h}{\partial x}\right)^{-1} \frac{\partial h}{\partial t},
$$

ou seja, $U(x, t)=\left(\frac{\partial h}{\partial x}\right)^{-1} \frac{\partial h}{\partial t}, x(t)$ deve ser solução da equação diferencial $\frac{d x}{d t}=-U(x, t)$. Assim a derivada parcial com relação a $t$ na forma Eureliana com $y=h(t, x)$ fixado corresponde à derivada anti-convectiva $D_{t}$ da forma Lagrangeana na região de referência $\Omega$

$$
D_{t}=\frac{\partial}{\partial t}-U(x, t) \frac{\partial}{\partial x}, U(x, t)=\left(\frac{\partial h}{\partial x}\right)^{-1} \frac{\partial h}{\partial t} .
$$

Mais precisamente, temos o seguinte. 
Lema 1.7 Suponha $\psi: \mathbb{R}^{n} \times \mathbb{R} \longrightarrow \mathbb{R}$ de classe $\mathcal{C}^{1}, h: \mathbb{R} \times \mathbb{R}^{n} \longrightarrow \mathbb{R}^{n}$ de classe $\mathcal{C}^{1}$ e $h(t, \cdot)$ um difeomorfismo de classe $\mathcal{C}^{1}$ sobre sua imagem $\Omega(t)$ para cada $t$. Então

$$
D_{t}\left(h^{*} \psi\right)=h^{*}\left(\frac{\partial \psi}{\partial t}\right)
$$

Prova. Para $0 \leq k, j \leq n$ sejam

$$
U=\left(\frac{\partial h}{\partial x}\right)^{-1} \frac{\partial h}{\partial t}=\left(U_{1}, \ldots, U_{n}\right) \text { onde } U_{k}=\sum_{j}\left(h_{x}^{-1}\right)_{k j} \frac{\partial h_{j}}{\partial t} \text { e } U \cdot \frac{\partial}{\partial x}=\sum_{k} U_{k} \cdot \frac{\partial}{\partial x_{k}} .
$$

Então temos

$$
\begin{aligned}
D_{t}\left(h^{*} \psi\right)(x) & =D_{t}(\psi(h(t, x), t)) \\
& =\frac{\partial \psi}{\partial t}+\sum_{j} \frac{\partial \psi}{\partial y_{j}} \frac{\partial h_{j}}{\partial t}-\sum_{k} U_{k} \frac{\partial}{\partial x_{k}} \psi(h(t, x), t) \\
& =\frac{\partial \psi}{\partial t}+\sum_{j} \frac{\partial \psi}{\partial y_{j}}\left(\frac{\partial h_{j}}{\partial t}-\sum_{k} U_{k} \frac{\partial h_{j}}{\partial x_{k}}\right) .
\end{aligned}
$$

Mas

$$
\begin{aligned}
\sum_{k} U_{k} \frac{\partial h_{j}}{\partial x_{k}} & =\sum_{k}\left(\sum_{i}\left(h_{x}^{-1}\right)_{k i} \frac{\partial h_{i}}{\partial t}\right) \frac{\partial h_{j}}{\partial x_{k}} \\
& =\sum_{i} \frac{\partial h_{i}}{\partial t} \sum_{k}\left(h_{x}^{-1}\right)_{k i}\left(\frac{\partial h_{j}}{\partial x_{k}}\right) \\
& =\sum_{i} \frac{\partial h_{i}}{\partial t} \delta_{j i} \\
& =\frac{\partial h_{j}}{\partial t}
\end{aligned}
$$

de onde segue o resultado.

Teorema 1.8 Suponha que $f(t, y, \lambda)$ é de classe $\mathcal{C}^{1}$ num conjunto aberto de $\mathbb{R} \times \mathbb{R}^{n} \times \mathbb{R}^{p}$, $L$ é um operador diferencial com coeficientes constantes de ordem menor ou igual a $m$ com $L v(y) \in \mathbb{R}^{p}$. Para um conjunto aberto $Q \subset \mathbb{R}^{n}$ e funções $v$ definidas em $Q$, seja $F_{Q}(t) v$ a aplicação

$$
y \longrightarrow f(t, y, L v(y)), y \in Q .
$$

Suponhamos que $t \longrightarrow h(t, \cdot)$ é uma curva de mergulhos de um conjunto aberto $\Omega \subset \mathbb{R}^{n}$, $\Omega(t)=h(t, \Omega)$ e para $|j| \leq m,|k| \leq m+1(t, x) \longrightarrow \partial_{t} \partial_{x}^{j} h(t, x), \partial_{x}^{k} h(t, x), \partial_{x}^{k} u(t, x)$ sejam contínuas em $\mathbb{R} \times \Omega$ numa vizinhança de $t=0$ e que $h(t, \cdot)^{*-1} u(t, \cdot)$ está no domínio de $F_{\Omega(t)}$. Então nos pontos de $\Omega$

$$
D_{t}\left(h^{*} F_{\Omega(t)}(t) h^{*-1}\right)(u)=\left(h^{*} \dot{F}_{\Omega(t)}(t) h^{*-1}\right)(u)+\left(h^{*} F_{\Omega(t)}^{\prime}(t) h^{*-1}\right)(u) \cdot D_{t} u
$$


onde $D_{t}$ é a derivada anti-convectiva definida anteriormente,

$$
\dot{F}_{Q}(t) v(y)=\frac{\partial f}{\partial t}(t, y, L v(y))
$$

$e$

$$
F_{Q}^{\prime}(t) v \cdot w(y)=\frac{\partial f}{\partial \lambda}(t, y, L v(y)) \cdot L w(y), y \in Q
$$

é a linearização de $v \longrightarrow F_{Q}(t) v$.

Prova. Defina como antes $v=h^{*-1} u$, isto é, $u(t, x)=v(t, h(t, x))$. Pelo lema (1.7)

$$
\begin{aligned}
h^{*-1} D_{t} u & =\left(D_{t} u\right) \circ h^{-1} \\
& =D_{t}\left(h^{*} v\right) \circ h^{-1} \\
& =\left(h^{*} \frac{\partial v}{\partial t}\right) \circ h^{-1} \\
& =\frac{\partial v}{\partial t} .
\end{aligned}
$$

Portanto

$$
\begin{aligned}
{\left[\left(h^{*} F_{\Omega(t)}^{\prime}(t) h^{*-1}\right)(u) \cdot D_{t} u\right](x) } & =\left[F_{\Omega(t)}^{\prime}(t)\left(u \circ h^{-1}\right) \cdot\left(D_{t} u\right) \circ h^{-1}\right](h(x)) \\
& =\left(F_{\Omega(t)}^{\prime}(t) v \cdot \frac{\partial v}{\partial t}\right)(y) \\
& =\frac{\partial f}{\partial \lambda}(t, y, L v(y)) \cdot \frac{\partial v}{\partial t}
\end{aligned}
$$

Agora

$$
\begin{aligned}
{\left[\left(h^{*} \dot{F}_{\Omega(t)}(t) h^{*-1}\right)(u)\right](x) } & =\dot{F}_{\Omega(t)}(t)\left(u \circ h^{-1}\right)(h(x)) \\
& =\dot{F}_{\Omega(t)}(t)(v)(y) \\
& =\frac{\partial f}{\partial t}(t, y, L v(y))
\end{aligned}
$$

logo, pelo lema (1.7) temos que

$$
\begin{aligned}
{\left[D_{t}\left(h^{*} F_{\Omega(t)}(t) h^{*-1}\right)(u)\right](x) } & =\left[h^{*}\left(\frac{\partial}{\partial t}\left(F_{\Omega(t)}(t) v\right)\right)\right](x) \\
& =\frac{\partial}{\partial t}[f(t, \cdot L v(\cdot))](h(x)) \\
& =\left(\frac{\partial f}{\partial t}(t, \cdot, L v(\cdot))+\frac{\partial f}{\partial \lambda}(t, \cdot, L v(\cdot)) \cdot\left[\frac{\partial v}{\partial t}\right)(h(x))\right. \\
& =\left[\left(h^{*} \dot{F}_{\Omega(t)}(t) h^{*-1}\right)(u)\right](x)+\left[\left(h^{*} F_{\Omega(t)}^{\prime}(t) h^{*-1}\right)(u) \cdot D_{t} u\right](x)
\end{aligned}
$$

de onde segue a afirmação.

Exemplos: 
1. Suponhamos que $A=\sum_{|\alpha| \leq m} a_{\alpha}(y)\left(\frac{\partial}{\partial y}\right)^{\alpha}$ é um operador diferencial linear que não depende explicitamente de $t$ e $h(t, x)=x+t V(x)+o(t)$ numa vizinhança de $t=0 \mathrm{e}$ $x \in \Omega$. Então pelo teorema (1.8) temos

$$
\begin{aligned}
\left.\frac{\partial}{\partial t}\left(h^{*} A h^{*-1} u\right)\right|_{t=0} & =\left.D_{t}\left(h^{*} A h^{*-1} u\right)\right|_{t=0}+\left.h_{x}^{-1} h_{t} \nabla\left(h^{*} A h^{*-1} u\right)\right|_{t=0} \\
& =A\left(\frac{\partial u}{\partial t}-V \cdot \nabla u\right)+V \cdot \nabla(A u) \\
& =A \frac{\partial u}{\partial t}+[V \cdot \nabla, A] u
\end{aligned}
$$

já que $\frac{\partial}{\partial t} A=0,[V \cdot \nabla, A](\cdot)$ é chamado comutador e ainda é uma operador de ordem $m$ embora $V \cdot \nabla A$ e $A V \cdot \nabla$ sejam operadores de ordem $m+1$. Portanto ainda pode ser calculado em funções de classe $\mathcal{C}^{m}$.

2. Dizemos que $\lambda_{0}$ é autovalor do problema de Dirichelet para o operador de Laplace $\Delta=\sum_{i=1}^{n} \frac{\partial^{2}}{\partial x_{i}^{2}}$ se existe solução não nula $u_{0}$ de

$$
\Delta u+\lambda_{0} u=0 \mathrm{em} \Omega, u=0 \mathrm{em} \partial \Omega
$$

onde $\Omega \subset \mathbb{R}^{n}$ é uma região $\mathcal{C}^{2}$-regular. Dizemos ainda que $\lambda_{0}$ é autovalor simples do problema de Dirichlet se $\operatorname{dim} \operatorname{ker}\left(\Delta+\lambda_{0}\right)=1$. Seja $u_{0}$ autofunção de (1.3) associada ao autovalor $\lambda_{0} \operatorname{com} \int_{\Omega} u_{0}^{2}=1$. Provaremos que em alguma vizinhança $\mathcal{C}^{2}$ de $i_{\Omega}, h(\Omega)$ é também uma região $\mathcal{C}^{2}$-regular e existe um único autovalor simples $\lambda_{h(\Omega)}$ próximo a $\lambda_{0}$ tal que a aplicação $h \longrightarrow \lambda_{h(\Omega)}$ é analítica. Isto segue facilmente do teorema da função implícita, de fato, defina

$$
\begin{aligned}
F: H^{2} \cap H_{0}^{1}(\Omega) \times \mathbb{R} \times \operatorname{Diff}^{3}(\Omega) & \longrightarrow L^{2}(\Omega) \times \mathbb{R} \\
(u, \lambda, h) & \left.\longrightarrow\left(h^{*}(\Delta+\lambda) h^{*-1} u, \int_{\Omega} u^{2}\right) \operatorname{let} h^{\prime}\right)
\end{aligned}
$$

$F\left(u_{0}, \lambda_{0}, i_{\Omega}\right)=(0,1)$ e sempre que $F(u, \lambda, h)=(0,1), v=h^{*-1} u$ satisfaz

$$
\Delta v+\lambda v=0 \operatorname{em} h(\Omega), v=0 \operatorname{em} \partial h(\Omega), \int_{h(\Omega)} v^{2}=1 .
$$

Observe que $F$ é analítica já que cada função coordenada de $F$ é analítica. A derivada

$$
\begin{aligned}
\frac{\partial F}{\partial(u, \lambda)}\left(u_{0}, \lambda_{0}, i_{\Omega}\right): H^{2} \cap H_{0}^{1}(\Omega) \times \mathbb{R} & \longrightarrow L^{2}(\Omega) \times \mathbb{R} \\
(\dot{u}, \dot{\lambda}) & \longrightarrow\left(\left(\Delta+\lambda_{0}\right) \dot{u}+\dot{\lambda} u_{0}, 2 \int_{\Omega} u_{0} \dot{u}\right)
\end{aligned}
$$

é um isomorfismo, de fato, sendo $\lambda_{0}$ um autovalor simples temos que, dado qualquer $(f, \xi) \in L^{2}(\Omega) \times \mathbb{R}$

$$
\left(\Delta+\lambda_{0}\right) v+\alpha u_{0}=f \text { e } 2 \int_{\Omega} u_{0} v=\xi
$$


tem solução única $(v, \alpha) \in H^{2} \cap H_{0}^{1}(\Omega) \times \mathbb{R}$

$$
\alpha=\int_{\Omega} u_{0} f \text { e } v=v_{0}+\frac{1}{2} \xi u_{0}
$$

onde $\left(\Delta+\lambda_{0}\right) v_{0}=f-\alpha u_{0}, v_{0} \perp u_{0}$ e $v_{0} \in H^{2} \cap H_{0}^{1}(\Omega)$. [Os detalhes da prova de que tal derivada é um isomorfismo podem ser vistos na seção (3.3).]

Então, pelo teorema da função implícita existem aplicações analíticas $h \longrightarrow u_{h} \mathrm{e}$ $h \longrightarrow \lambda_{h}$ numa vizinhança $\mathcal{C}^{2}$ de $i_{\Omega}$ tal que $F\left(u_{h}, \lambda_{h}, h\right)=(0,1)$, de onde segue a afirmação. 
CAPÍtulo 2

\section{Operadores de Fredholm e o Teorema da Transversalidade}

Na primeira parte deste capítulo estudaremos os operadores de Fredholm e semi-Fredholm obtendo algumas propriedades importantes que serão utilizadas no capítulo de aplicações.

Na segunda parte enunciaremos o Teorema da Transversalidade, que será a principal ferramenta utilizada nas aplicações.

\subsection{Operadores de Fredholm}

Neste capítulo $X, Y$ e $Z$ denotarão sempre espaços de Banach a não ser que haja menção contrária. Dado qualquer elemento $x \in X$, a norma de $x$ em $X$ será denotada por $\|x\|_{X}$. Um operador linear, ou simplesmente um operador $T: X \longrightarrow Y$ é contínuo em $x \in X$ se $\left\|x_{n}-x\right\|_{X} \longrightarrow 0, x_{n} \in X$, implica que $\left\|T x_{n}-T x\right\|_{Y} \longrightarrow 0$ quando $n \longrightarrow+\infty$. $T$ é contínuo em todo o domínio $X$ se é contínuo em $x=0$ e $T$ é contínuo se somente se $T$ é limitado, ou seja, $\|T x\|_{Y} \leq M\|x\|_{X} \forall x \in X$. O menor número $M$ com esta propriedade é a norma de $T$ que será indicada por $\|T\|$. Denotamos por $\operatorname{ker} T$ o núcleo do operador $T$ e $\operatorname{Im} T$ a sua imagem. Dado um subespaço $M$ qualquer de um espaço de Banach, chamamos de codimensão de $M$ a $\operatorname{dim} X / M$ que é denotada por codim $M$. Denotamos ainda por $\mathcal{B}(X, Y)$ o conjunto de todos os operadores lineares contínuos com domínio $X$ e contra-domínio $Y . \mathcal{B}(X, Y)$ é um espaço vetorial normado com norma $\|T\|_{\mathcal{B}(X, Y)}$ acima. $\mathrm{O}$ produto (composição) $T S \operatorname{com} T \in \mathcal{B}(Y, Z)$ e $S \in \mathcal{B}(X, Y)$ pode ser definido e pertence a $\mathcal{B}(X, Z)$.

Dizemos que $T$ é Fredholm se $\operatorname{ImT}$ é fechada e $\operatorname{dim} \operatorname{ker} T$ e codim $\operatorname{Im} T$ são ambas finitas. Neste caso

$$
\operatorname{ind}(T)=\operatorname{dim} \operatorname{ker} T \bar{\oplus} \operatorname{codim} \operatorname{Im} T
$$


que é um número inteiro. Se $\operatorname{Im} T$ é fechada e ao menos $\operatorname{dim} \operatorname{ker} T$ ou codim $\operatorname{Im} T$ é finita, $T$ é semi-Fredholm e o ind(T) é $+\infty$ ou $-\infty$ respectivamente.

\section{Exemplos:}

1. Se $X$ e $Y$ são espaços de Banach de dimensão finita, todo operador $T: X \longrightarrow Y$ é Fredholm com $i n d(T)=\operatorname{dim} X-\operatorname{dim} Y$. De fato, se o posto de $T$ é igual a $r$ a $\operatorname{dim} \operatorname{ker} T=\operatorname{dim} X-r$ e a codim $\operatorname{Im} T=\operatorname{dim} Y-r$. Se ao menos um dos $X, Y$ é de dimensão finita, todo operador contínuo é semi-Fredholm e $i n d(T)=\operatorname{dim} X-\operatorname{dim} Y$ como no caso anterior.

2. Suponha $X$ e $Y$ espaços de Banach de dimensão infinita. O operador nulo não é semiFredholm e qualquer outro operador compacto de $X$ para $Y$ não é semi-Fredholm. De fato, se $T$ é um operador compacto com $\operatorname{Im} T$ fechada, temos que $\operatorname{dim} \operatorname{Im} T<\infty$. Daí como $\operatorname{dim} Y$ é infinita temos que a codim $\operatorname{Im} T$ é infinita. Observe que a $\operatorname{dim} X$ ser infinita e a $\operatorname{dim} \operatorname{Im} T<\infty$ com $\operatorname{Im} T$ fechada implica que $\operatorname{dim} \operatorname{ker} T$ é infinita pois se não existiria um isomorfismo entre um espaço de dimensão finita e um espaço de dimensão infinita. Logo $T$ compacto não pode ser semi-Fredholm.

3. Seja $X=Y=l^{2}$ o conjunto de todas as sequências $x=\left(x_{1}, x_{2}, \ldots, x_{n}, \ldots\right)$ de números reais tais que $\sum_{i=1}^{+\infty} x_{i}^{2}<\infty$. A identidade em $l^{2}$ ou qualquer isomorfismo é Fredholm de índice zero. A translação $S:\left(x_{1}, x_{2}, \ldots, x_{n}, \ldots\right) \longrightarrow\left(x_{2}, x_{3} \ldots, x_{n}, \ldots\right)$ é Fredholm com índice 1 e $S^{m}$ é Fredholm com índice $m$. O adjunto $S^{*}:\left(x_{1}, x_{2}, \ldots, x_{n}, \ldots\right) \longrightarrow$ $\left(0, x_{1}, x_{2}, \ldots, x_{n}, \ldots\right)$ é Fredholm com índice -1 e $\left(S^{*}\right)^{m}$ é Fredholm com índice $-m$. $L:\left(x_{1}, x_{2}, \ldots, x_{n}, \ldots\right) \longrightarrow\left(x_{1}, 0, x_{2}, 0, \ldots\right)$ é Fredholm de índice $-\infty$ e seu adjunto $L^{*}:\left(x_{1}, x_{2}, \ldots, x_{n}, \ldots\right) \longrightarrow\left(x_{1}, x_{3}, x_{5}, \ldots\right)$ é semi-Fredholm de índice $+\infty$. Note que $L^{*} L$ é a identidade que é Fredholm de índice zero, mas $L L^{*}$ não é semi-Fredholm.

Vale a pena observar também que se a $\operatorname{dim} \operatorname{ker} T$ e a codim $\operatorname{Im} T$ são finitas, podemos provar, em vez de assumir, que $\operatorname{Im} T$ é fechada. De fato, seja $X=X_{1} \oplus X_{2}, X_{1}=k e r T$ e $X_{2}$ seu complementar fechado e seja $Y_{1}$ um subespaço de dimensão finita tal que $Y=Y_{1} \oplus \operatorname{Im} T$. Defina $G: Y_{1} \times X_{2} \longrightarrow Y:\left(y_{1}, x_{2}\right) \longrightarrow y_{1}+T x_{2}$. G é um isomorfismo, $y_{1}+T x_{2}=0$ se somente se $y_{1} \in \operatorname{Im} T$ o que nos dá $\left(y_{1}, x_{2}\right)=(0,0)$ e $G$ é claramente sobrejetora. Portanto $G$ é uma bijeção e pelo teorema do Gráfico Fechado é um isomorfismo. Logo, como $0 \times X_{2}$ é fechado e $G$ é um isomorfismo, $G\left(0 \times X_{2}\right)=\operatorname{ImT}$ é fechado.

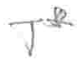

Teorema 2.1 $T \in \mathcal{B}(X, Y)$ é semi-Fredholm se somente se $\mathcal{B}\left(X^{*}, Y^{*}\right)$ é semi-Fredholm. Neste caso ind $(T)=-$ ind $\left(T^{*}\right)$.

Prova. De fato, pelo teorema 5.13 em [14] temos que a $\operatorname{ImT}$ é fechada se somente se 
$\operatorname{Im} T^{*}$ é fechada e neste caso $\operatorname{Im} T^{\perp}=k e r T^{*}, \operatorname{ker} T^{\perp}=\operatorname{Im} T^{*}, \operatorname{dim} \operatorname{ker} T^{*}=\operatorname{codim} \operatorname{Im} T$ e codim $\operatorname{Im} T^{*}=\operatorname{dim} \operatorname{ker} T$, de onde o resultado segue imediatamente.

Teorema 2.2 Se $T \in \mathcal{B}(X, Y)$ é semi-Fredholm, existe $\delta>0$ tal que se $\|S-T\|_{\mathcal{B}(X, Y)}<\delta$, $S$ é semi-Fredholm e o ind $(S)=$ ind $(T)$. Ou seja, o conjunto dos operadores semi-Fredholm com dado índice é aberto em $\mathcal{B}(X, Y)$.

Prova. Suponha inicialmente que $T$ é Fredholm. Então $X_{1}=\operatorname{ker} T$ e $Y_{2}=\operatorname{Im} T$ são subespaços fechados. De $\operatorname{dim} \operatorname{ker} T<\infty$ e $\operatorname{codim} \operatorname{Im} T<\infty$ temos $X=X_{1} \oplus X_{2}$ para algum subespaço fechado $X_{2}$ de $X$ e temos $Y=Y_{1} \oplus Y_{2}$ para algum subespaço fechado de dimensão finita $Y_{1} \subset Y$. Feita tal decomposição, todo $S \in \mathcal{B}(X, Y)$ pode ser escrito na forma matricial

$$
S=\left(\begin{array}{ll}
S_{11} & S_{12} \\
S_{21} & S_{22}
\end{array}\right):\left(\begin{array}{c}
X_{1} \\
X_{2}
\end{array}\right) \rightarrow\left(\begin{array}{c}
Y_{1} \\
Y_{2}
\end{array}\right):\left(\begin{array}{c}
x_{1} \\
x_{2}
\end{array}\right) \longrightarrow\left(\begin{array}{c}
S_{11} x_{1}+S_{12} x_{2} \\
S_{21} x_{1}+S_{22} x_{2}
\end{array}\right)
$$

onde $S_{i j} \in \mathcal{B}\left(X_{j}, Y_{i}\right)$ com $i, j=1,2$. Em particular

$$
T=\left(\begin{array}{ll}
T_{11} & T_{12} \\
T_{21} & T_{22}
\end{array}\right)
$$

Agora $T_{11} x_{1}+T_{12} x_{2}=0 \forall x \in X$ pois $\operatorname{Im} T=Y_{2}$, daí $T_{11} \equiv T_{12} \equiv 0 . T\left(X_{1}\right)=\{0\} \Longrightarrow$ $T_{21} x_{1}=0, \forall x_{1} \in X_{1}$, então $T_{21} \equiv 0$. Logo

$$
T=\left(\begin{array}{ll}
0 & 0 \\
0 & T_{22}
\end{array}\right)
$$

onde $T_{22}: X_{2} \longrightarrow Y_{2}$ é isomorfismo. Qualquer $T_{1}$ próximo a $T$ pode ser escrito na forma

$$
T_{1}=\left(\begin{array}{ll}
A & B \\
C & T_{22}+D
\end{array}\right)
$$

com $\|A\|_{\mathcal{B}\left(X_{1}, Y_{1}\right)},\|B\|_{\mathcal{B}\left(X_{2}, Y_{1}\right)},\|C\|_{\mathcal{B}\left(X_{1}, Y_{2}\right)}$ e $\|D\|_{\mathcal{B}\left(X_{2}, Y_{2}\right)}$ pequenas. Se $\left\|T-T_{1}\right\|_{\mathcal{B}(X, Y)}<\epsilon$ temos que $\|D\|_{\mathcal{B}\left(X_{2}, Y_{2}\right)}<c_{0} \epsilon$ onde $c_{0}$ é uma constante que depende apenas das projeções em $X_{2}$ e $Y_{2}$. Daí como $T_{22}$ é um isomorfismo e o conjunto dos isomorfismos é aberto, temos que $T_{22}+D$ é também um isomorfismo. Seja

$$
\tilde{T}_{1}=\left(\begin{array}{ll}
I & P \\
0 & I
\end{array}\right) T_{1}\left(\begin{array}{ll}
I & 0 \\
Q & I
\end{array}\right)
$$

com $P=-B\left(T_{22}+D\right)^{-1}, Q=-\left(T_{22}+D\right)^{-1} C$ e $I$ a identidade. Note que

$$
\left(\begin{array}{ll}
I & -P \\
0 & I
\end{array}\right)=\left(\begin{array}{ll}
I & P \\
0 & I
\end{array}\right)^{-1} \text { e }\left(\begin{array}{ll}
I & 0 \\
-Q & I
\end{array}\right)=\left(\begin{array}{ll}
I & 0 \\
Q & I
\end{array}\right)^{-1} .
$$


Portanto

$$
\left(\begin{array}{ll}
I & P \\
0 & I
\end{array}\right) \text { e }\left(\begin{array}{ll}
I & 0 \\
Q & I
\end{array}\right)
$$

são isomorfismos de $Y$ em $Y$ e de $X$ em $X$ respectivamente e $\operatorname{ind}\left(\tilde{T}_{1}\right)=\operatorname{ind}\left(T_{1}\right)$. Agora, fazendo a multiplicação obtemos

$$
\tilde{T}_{1}=\left(\begin{array}{ll}
\Delta & 0 \\
0 & T_{22}+D
\end{array}\right)
$$

com $\Delta=A-B\left(T_{22}+D\right)^{-1} C \in \mathcal{B}\left(X_{1}, Y_{1}\right)$. Observe agora que

$$
\operatorname{Im} \tilde{T}_{1}=\left(\begin{array}{l}
\operatorname{Im} \Delta \\
Y_{2}
\end{array}\right) \operatorname{e~} \operatorname{ker} \tilde{T}_{1}=\left(\begin{array}{l}
k e r \Delta \\
0
\end{array}\right)
$$

portanto, temos que a codim $\operatorname{Im} \tilde{T}_{1}=\operatorname{codim} \operatorname{Im} \Delta$ e que $\operatorname{dim} \operatorname{ker} \tilde{T}_{1}=\operatorname{dim} \operatorname{ker} \Delta$. Logo $\tilde{T}_{1}$ é Fredholm e portanto $T_{1}$ é Fredholm com $i n d\left(T_{1}\right)=i n d\left(\tilde{T}_{1}\right)=i n d(\Delta)=\operatorname{dim} X_{1}-\operatorname{dim} Y_{1}=$ $\operatorname{ind}(T)$ já que $\Delta \in \mathcal{B}\left(X_{1}, Y_{1}\right)$ com $X_{1}$ e $Y_{1}$ espaços de dimensão finita.

Suponha agora $T$ semi-Fredholm com índice $-\infty$. Como no caso anterior, seja $X=$ $X_{1} \oplus X_{2} \operatorname{com} X_{1}=k e r T$. Seja $Y_{2}=\operatorname{Im} T$ que é fechada e possui codimensão infinita. A restrição de $T$ a $\mathcal{B}\left(X_{2}, Y_{2}\right)$ é uma bijeção contínua e então, pelo teorema do gráfico fechado, existe $T^{-1} \in \mathcal{B}\left(Y_{2}, X_{2}\right)$ o que implica $\left\|T x_{2}\right\|_{Y} \geq c\left\|x_{2}\right\|_{X}$ para algum $c>0$ e $\forall x_{2} \in X_{2}$. Vamos mostrar que qualquer $T_{1}$ com $\left\|T-T_{1}\right\|_{\mathcal{B}(X, Y)}<\frac{c}{5}$ é semi-Fredholm com índice $-\infty$. Suponha $x=x_{1}+x_{2} \in \operatorname{ker} T_{1}$. Então, se $\Delta=T_{1}-T$ com $\|\Delta\|_{\mathcal{B}(X, Y)} \leq \frac{c}{5}$ temos que $T x_{2}+\Delta\left(x_{1}+x_{2}\right)=0$. Assim

$$
c\left\|x_{2}\right\|_{X} \leq\left\|T x_{2}\right\|_{Y} \leq\|\Delta\|_{\mathcal{B}(X, Y)}\left(\left\|x_{1}\right\|_{X}+\left\|x_{2}\right\|_{X}\right) \Longrightarrow\left\|x_{2}\right\|_{X} \leq \frac{\|\Delta\|_{\mathcal{B}(X, Y)}}{c+\|\Delta\|_{\mathcal{B}(X, Y)}}\left\|x_{1}\right\|_{X} .
$$

De tal desigualdade podemos provar que $\operatorname{ker} T_{1}$ está contido num gráfico sobre $X_{1}$ o que nos dá $\operatorname{dim} \operatorname{ker} T_{1}<\infty$. Com efeito, seja $P: X \longrightarrow X$ a projeção sobre $X_{1}$ e defina

$$
\varphi: P\left(k e r T_{1}\right) \longrightarrow X_{2}: P x \longrightarrow(I-P) x .
$$

$\varphi\left(x^{\prime}+\lambda y^{\prime}\right)=\varphi(P x+\lambda P y)=\varphi(P(x+\lambda y))=(I-P)(x+\lambda y)=\varphi\left(x^{\prime}\right)+\lambda \varphi\left(y^{\prime}\right)$ para $x^{\prime}$, $y^{\prime} \in P\left(\operatorname{ker} T_{1}\right)$, o que implica $\varphi$ linear. Como $P\left(\operatorname{ker} T_{1}\right) \subset X_{1}$ que tem dimensão finita, $\varphi$ é contínua. Observe agora que $\varphi$ está bem definida, de fato, dados $x^{\prime}, y^{\prime} \in P\left(k e r T_{1}\right)$ tal que $x^{\prime}=y^{\prime}$, existem $x$ e $y \in k e r T_{1}, x=x^{\prime}+x_{2}$ e $y=y^{\prime}+y_{2}$ para $x_{2}$ e $y_{2} \in X_{2}$. Pela desigualdade (2.1), já que $x-y \in \operatorname{ker} T_{1}$,

$$
0 \leq\left\|x_{2}-y_{2}\right\|_{X} \leq \frac{\|\Delta\|_{\mathcal{B}(X, Y)}}{c+\|\Delta\|_{\mathcal{B}(X, Y)}}\left\|x^{\prime}-y^{\prime}\right\|_{X}=0,
$$


portanto $\varphi\left(x^{\prime}\right)=x_{2}=y_{2}=\varphi\left(y^{\prime}\right)$, ou seja, $\varphi$ está bem definida. Mostraremos agora que $\operatorname{Im} T_{1}$ é fechada. Seja então $y \neq 0 \in \operatorname{Im} T_{1}$ e $x^{n} \in X$ tal que $T_{1} x^{n} \longrightarrow y$ quando $n \longrightarrow+\infty$. Já que $y \neq 0$ podemos supor que $x^{n} \notin k e r T_{1}$, mais ainda, podemos supor que $\operatorname{dist}\left(\frac{x^{n}}{\left\|x^{n}\right\|_{X}}, \operatorname{ker} T_{1}\right) \geq \frac{1}{2} \Longrightarrow \operatorname{dist}\left(x^{n}, \operatorname{ker} T_{1}\right) \geq \frac{1}{2}\left\|x^{n}\right\|_{X} \forall n \in \mathbb{N}$. Se $\left\{x^{n}\right\}$ é limitada, já que a $\operatorname{dim} X_{1}<\infty, X_{1}$ é localmente compacto e portanto podemos supor que $\left\{x_{1}^{n}\right\} \subset X_{1}$ converge. Agora

$$
c\left\|x_{2}^{n}-x_{2}^{m}\right\|_{X} \leq\left\|T\left(x_{2}^{n}-x_{2}^{m}\right)\right\|_{Y} \leq\left\|T_{1}\left(x_{2}^{n}-x_{2}^{m}\right)\right\|_{Y}+\|\Delta\|_{\mathcal{B}(X, Y)}\left\|x_{2}^{n}-x_{2}^{m}\right\|_{X}
$$

o que implica

$$
\left\|x_{2}^{n}-x_{2}^{m}\right\|_{X} \leq \frac{1}{c-\|\Delta\|_{\mathcal{B}(X, Y)}}\left\|T_{1}\left(x_{2}^{n}-x_{2}^{m}\right)\right\|_{Y}
$$

que tende a 0 quando $n, m \longrightarrow+\infty$, de onde podemos concluir que $\left\{x_{2}^{n}\right\}$ também converge e então $\left\{x^{n}\right\}$ converge para algum $x \in X$. Logo, como $T_{1}$ é contínuo, $T_{1} x^{n} \longrightarrow T_{1} x=y \Longrightarrow$ $\operatorname{Im} T_{1}$ é fechada. Agora suponha que $\left\{x^{n}\right\}$ não é limitada, ou seja, passando a subsequência $\left\|x^{n}\right\|_{X} \longrightarrow+\infty$. A componente da sequência $\left\{\frac{x^{n}}{\left\|x^{n}\right\|_{X}}\right\}$ em $X_{1}$, passando a subsequência, converge e isto, como no caso anterior, implica na convergência de $\frac{x^{n}}{\left\|x^{n}\right\|_{X}}$ para algum $u \in X$ tal que $\|u\|_{X}=1$. Então $T_{1} \frac{x^{n}}{\left\|x^{n}\right\|_{X}} \longrightarrow T_{1} u=0$ pois $T_{1} x^{n} \longrightarrow y \neq 0$ e $\left\|x^{n}\right\|_{X} \longrightarrow \infty$ quando $n \longrightarrow+\infty$. Mas, por construção, $\operatorname{dist}\left(\frac{x^{n}}{\left\|x^{n}\right\|_{X}}, k \operatorname{er} T_{1}\right) \geq \frac{1}{2}$, contradição. Portanto $\operatorname{Im} T_{1}$ é fechada. Mostraremos agora que a codim $T_{1}=\infty$. Sendo a codim $T=\infty$ podemos definir uma sequência $\left\{y_{n}\right\} \subset Y$ com $\left\|y_{n}\right\|_{Y}=1$, $\operatorname{dist}\left(y_{n},\left[\operatorname{Im} T \cup\left\{y_{1}, \ldots, y_{n-1}\right\}\right]\right) \geq \frac{1}{2}$ ([ ] significa subespaço gerado). Suponha que $\operatorname{Im} T_{1}$ tem codimensão finita. Então $Y=T_{1}\left(X_{2}\right) \oplus \tilde{Y}_{2}$ para algum subespaço $\tilde{Y}_{2}$ de dimensão finita. Desta maneira todo ponto de $Y$ pode ser escrito como $\tilde{y}+T_{1} x^{2}, \tilde{y} \in \tilde{Y_{2}}$ e $x^{2} \in X_{2}$. Assim $y_{n}=\tilde{y_{n}}+T_{1} x_{n}^{2} \forall n \in \mathbb{N}$. Observe que $\left\{\tilde{y_{n}}\right\}$ é limitada num espaço de dimensão finita, portanto possui subsequência convergente. Passando a subsequência temos como em (2.2)

$$
\begin{aligned}
\left\|x_{2}^{n}-x_{2}^{m}\right\|_{X} & \leq \frac{1}{c-\|\Delta\|_{\mathcal{B}(X, Y)}}\left\|T_{1}\left(x_{2}^{n}-x_{2}^{m}\right)\right\|_{Y} \\
& \leq \frac{1}{c-\|\Delta\|_{\mathcal{B}(X, Y)}}\left(2+\left\|\tilde{y_{n}}-\tilde{y_{m}}\right\|_{Y}\right) \\
& \leq \frac{2}{c-\|\Delta\|_{\mathcal{B}(X, Y)}}
\end{aligned}
$$

se $n, m \longrightarrow+\infty$. Temos também para $n>m$, pela definição de $\left\{y_{n}\right\}$

$$
\begin{aligned}
\frac{1}{2} & \leq\left\|y_{n}-y_{m}-T\left(x_{n}^{2}-x_{m}^{2}\right)\right\|_{Y} \\
& \leq\left\|\tilde{y_{n}}-\tilde{y_{m}}+T_{1}\left(x_{n}^{2}-x_{m}^{2}\right)-T\left(x_{n}^{2}-x_{m}^{2}\right)\right\|_{Y} \\
& \leq\left\|\tilde{y_{n}}-\tilde{y_{m}}+\Delta\left(x_{n}^{2}-x_{m}^{2}\right)\right\|_{Y} \\
& \leq\|\Delta\|_{\mathcal{B}(X, Y)}\left\|x_{n}^{2}-x_{m}^{2}\right\|_{X}
\end{aligned}
$$


se $m \longrightarrow+\infty(n>m)$. Assim

$$
\frac{1}{2} \leq \frac{2\|\Delta\|_{\mathcal{B}(X, Y)}}{c-\|\Delta\|_{\mathcal{B}(X, Y)}} \Longrightarrow c \leq 5\|\Delta\|_{\mathcal{B}(X, Y)}
$$

mas nós assumimos que $\|\Delta\|_{\mathcal{B}(X, Y)}<\frac{c}{5}$, contradição. Assim codim $\operatorname{Im} T_{1}=+\infty$ o que nos dá ind $\left(T_{1}\right)=-\infty$.

Finalmente se $T$ é semi-Fredholm com índice $+\infty, T^{*}$ pelo teorema (2.1) é semiFredholm com índice $-\infty$. Agora para todo $T_{1}$ próximo a $T, T_{1}^{*}$ está próximo a $T^{*} \mathrm{e}$ é semi-Fredholm com índice $-\infty$ pelo que provamos anteriormente. Então, novamente pelo teorema (2.1) temos que $T_{1}$ é semi-Fredholm com índice $+\infty$ de onde segue o teorema.

Teorema 2.3 Sejam $T, K \in \mathcal{B}(X, Y)$ com $T$ semi-Fredholm e $K$ compacto. Então $T+K$ é semi-Fredholm com ind $(T+K)=$ ind $(T)$.

Prova. Suponha que $i n d(T)<+\infty$ (podemos ter $i n d(T)=-\infty$ ). Então $X=X_{1} \oplus X_{2}$ onde $X_{1}=\operatorname{ker} T$ que possui dimensão finita. A restrição $\left.T\right|_{X_{2}} \longrightarrow \operatorname{Im} R(T)$ é um isomorfismo, portanto $\left\|T x_{2}\right\|_{Y} \geq c\left\|x_{2}\right\|_{X} \forall x_{2} \in X_{2}$ e algum $c>0$.

Afirmamos que $\operatorname{dim} \operatorname{ker}(T+K)<+\infty$, de fato, tome uma sequência $\left\{x_{n}\right\} \subset \operatorname{ker}(T+K)$, $x_{n}=x_{n}^{1}+x_{n}^{2}$ e $\left\|x_{n}\right\|_{X}=1 \forall n \in \mathbb{N}$. $\left\{x_{n}^{1}\right\} \subset X_{1}$ e $\left\{K x_{n}\right\}$ possuem subsequências convergentes pois $\left\{x_{n}\right\}$ é limitada, $X_{1}$ é localmente compacto e $K$ é um operador compacto. Então como $(T+K) x_{n}=0 \forall n$ temos que $T x_{n}^{2}=-K x_{n}$ é uma sequência convergente. Como $T$ é Fredholm, $T$ possui imagem fechada e então $T x_{n}^{2}$ converge para algum $T x$ na imagem de $T$. Daí como $\left\|T x_{2}\right\|_{Y} \geq c\left\|x_{2}\right\|_{X} \forall x_{2} \in X_{2}$ temos que a sequência $\left\{x_{n}^{2}\right\}$ converge para $x \in X$. Portanto $\left\{x_{n}\right\}$ possui subsequência convergente implicando que $\operatorname{ker}(T+K)$ é localmente compacto. Logo, como o $\operatorname{ker}(T+K)$ é também fechado, tal subespaço possui dimensão finita.

Mostraremos agora que $\operatorname{Im}(T+K)$ é fechada. Suponha que a sequência $(T+K) x_{n} \longrightarrow y$ $\operatorname{com} \operatorname{dist}\left(x_{n}, \operatorname{ker}(T+K)\right) \geq \frac{1}{2}\left\|x_{n}\right\|_{X} \forall n \in \mathbb{N}$ como na prova do teorema (2.2). Se $\left\{x_{n}\right\}$ é limitado, podemos assumir que $\left\{x_{n}^{1}\right\}$ e $\left\{K x_{n}\right\}$ são convergentes e assim obter $\left\{x_{n}^{2}\right\}$ convergente como acima e então concluiremos que $\left\{x_{n}\right\}$ converge para algum $x \in X$ o que nos dá $(T+K) x=y$, ou seja, $\operatorname{Im}(T+K)$ é fechada. Agora se $\left\{x_{n}\right\}$ não é limitada, passando a subsequência podemos assumir $\left\|x_{n}\right\|_{X} \longrightarrow+\infty$. A sequência $\left\{\frac{x_{n}}{\left\|x_{n}\right\|_{X}}\right\}$ é limitada, portanto as sequências $\left\{K \frac{x_{n}}{\left\|x_{n}\right\|_{X}}\right\}$ e $\left\{\frac{x_{n}^{1}}{\left\|x_{n}\right\|_{X}}\right\}$ possuem subsequência convergente implicando $\left\{T \frac{x_{n}^{2}}{\left\|x_{n}\right\|_{X}}\right\}$ convergente que nos dá $\left\{\frac{x_{n}^{2}}{\left\|x_{n}\right\|_{X}}\right\}$ convergente pois $\left\|T x_{2}\right\|_{Y} \geq c\left\|x_{2}\right\|_{X} \forall x_{2} \in X_{2}$. Portanto, passando a subsequência, a sequência $\left\{\frac{x_{n}}{\left\|x_{n}\right\|_{X}}\right\}$ converge para algum $u \in X$ onde $\|u\|_{X}=1$. Então $(T+K) \frac{x_{n}}{\left\|x_{n}\right\|_{X}} \longrightarrow(T+K) u=0$ pois $(T+K) x_{n} \rightarrow y$ e $\left\|x_{n}\right\|_{X} \longrightarrow+\infty$, contradição, já que por construção $\operatorname{dist}\left(x_{n}, \operatorname{ker}(T+K)\right) \geq \frac{1}{2}\left\|x_{n}\right\|_{X} \forall n \in \mathbb{N}$. Logo $\operatorname{Im}(T+K)$ é fechada. 
Temos então que $T+K$ é um operador semi-Fredholm como é o operador $T+\alpha K$ para todo $\alpha \in[0,1]$. Pelo teorema (2.2) a aplicação $\psi: \alpha \longrightarrow i n d(T+\alpha K)$ é contínua e localmente constante. Como $[0,1]$ é conexo temos que $\psi$ é uma aplicação constante, de onde podemos concluir que $i n d(T)=i n d(T+K)$.

Suponha agora que $T$ é apenas Fredholm, pela afirmação anterior

$$
\operatorname{ind}(T)=\operatorname{ind}(T+K)=\operatorname{dim} \operatorname{ker}(T+K)-\operatorname{codim} \operatorname{Im}(T+K)
$$

que é finito. Portanto temos que codim $\operatorname{Im}(T+K)$ é finito o que implica $T+K$ um operador de Fredholm.

O caso em que o ind $(T)=+\infty$ nos dá pelo teorema (2.1) que o $\operatorname{ind}\left(T^{*}\right)=-\infty$. Daí pelo que foi mostrado anteriormente temos que $T^{*}+K^{*}$ é semi-Fredholm com índice $-\infty$ o que implica novamente pelo teorema (2.1) que $T+K$ é semi-Fredholm com índice $+\infty$ de onde segue o teorema.

Corolário 2.4 Se $K \in \mathcal{B}(X, Y)$ é compacto, $I+K$ e $I^{*}+K^{*}$ são operadores Fredholm com índice zero. Assim se $I+K$ ou $I^{*}+K^{*}$ é injetivo, ambos são isomorfismos. Em geral temos

$\operatorname{dim} \operatorname{ker}(I+K)=\operatorname{codim} \operatorname{Im}(I+K)=\operatorname{dim} \operatorname{ker}\left(I^{*}+K^{*}\right)=\operatorname{codim} \operatorname{Im}\left(I^{*}+K^{*}\right)$ que é finita.

Prova. Segue diretamente do teorema anterior.

Embora o próximo resultado não vá ser usado no que segue, ele é uma propriedade básica dos operadores de Fredholm (ou semi-Fredholm).

Teorema 2.5 Se $S \in \mathcal{B}(X, Y)$ e $T \in \mathcal{B}(Y, Z)$ são semi-Fredholm e se o ind $(S)$ e o ind $(T)$ podem ser somados, então $T S \in \mathcal{B}(X, Z)$ é semi-Fredholm com ind $(T S)=\operatorname{ind}(T)+i n d(S)$.

Prova. Inicialmente assumiremos $T$ e $S$ sobrejetor ou injetor.

$T$ e $S$ sobrejetores Sendo $T$ e $S$ sobrejetores temos que $T S$ é sobrejetor com $\operatorname{ker}(T S)=$ $S^{-1}(\operatorname{ker}(T))$, daí $\operatorname{dim} \operatorname{ker}(T S)=\operatorname{dim} \operatorname{ker} S+\operatorname{dim} \operatorname{ker} T$ finito ou não. Assim ind $(T S)=$ $\operatorname{ind}(T)+i n d(S)$. Observe que $\operatorname{Im}(T S)$ é obviamente fechada já que $T S$ é sobrejetor.

$T$ e $S$ injetores $T$ e $S$ injetores implica $T S$ injetor. Observemos que a $\operatorname{Im}(T S)$ é fechada pois $\|T S x\|_{Y} \geq c\|x\|_{X} \forall x \in X$ para algum $c>0$ já que os operadores $T$ e $S$ satisfazem o mesmo tipo de estimativa. Provermos que a codim $\operatorname{Im}(T S)=\operatorname{codim} \operatorname{Im}(T)+$ 
codim $\operatorname{Im}(S)$ finita ou não, de onde segue o teorema. Suponha que $T$ e $S$ são operadores Fredholm, caso contrário a codimensão da imagem de um dos operadores é infinita e a afirmação segue facilmente pois ambos os operadores são injetores. Então $Y=Y_{1} \oplus Y_{2}$ onde $Y_{1}=\operatorname{ImS}$ e $Y_{2}$ é um subespaço de dimensão finita e $Z=Z_{1} \oplus Z_{2}$ onde $Z_{1}=\operatorname{Im} T$ e $Z_{2}$ é um subespaço de dimensão finita. Sendo $T$ injetivo e a $\operatorname{dim} Y_{2}$ finito temos que $Z=Z_{1} \oplus Z_{2}=T(\operatorname{Im} S) \oplus T\left(Y_{2}\right) \oplus Z_{2}$ onde a $\operatorname{dim} Y_{2}=\operatorname{dim} T\left(Y_{2}\right)$. Então codim $\operatorname{Im}(T S)=\operatorname{dim} T\left(Y_{2}\right)+\operatorname{dim} Z_{2}=\operatorname{codim} \operatorname{Im}(T)+\operatorname{codim} \operatorname{Im}(S)$.

$T$ injetora e $S$ sobrejetora $\operatorname{ker}(T S)=k e r S$ e $\operatorname{Im}(T S)=\operatorname{Im}(T)$, então $\operatorname{Im}(T S)$ é fechada e se ao menos um dos $T$ e $S$ possui índice finito, os índices podem ser somados e $T S$ é semi-Fredholm com índice $\operatorname{ind}(T)+i n d(S)$.

$T$ sobrejetora e $S$ injetora Observe que ao menos um dos operadores deve ter índice finito. Se ind $(T)=+\infty$, codim ImS é finita o que implica codim ImTS finita, de onde segue que a ImTS é fechada. Então TS é semi-Fredholm com índice $+\infty$. Se $i n d(T)$ é finito, $\operatorname{dim} \operatorname{ker} T=i n d(T)$ é finita. Daí se $Y_{1}=\operatorname{ker} T \cap \operatorname{Im} S, \operatorname{ker}(T S)=$ $S^{-1}\left(Y_{1}\right)$ que tem dimensão finita igual a $\operatorname{dim} Y_{1}$ já que $S$ é injetora. Escolha $Y_{2}$ tal que $Y_{1} \oplus Y_{2}=k e r T$. Temos então que $\operatorname{Im}(T S)=T(\operatorname{Im} S+k e r T)=T\left(\operatorname{Im} S \oplus Y_{2}\right)$ de onde segue que a codim $\operatorname{Im}(T S)=\operatorname{codim} \operatorname{Im} S-\operatorname{dim} Y_{2}$ já que o núcleo de $T$ está contido na $\operatorname{Im} S \oplus Y_{2}$. Assim $\operatorname{ind}(T S)=\operatorname{dim} Y_{1}+\operatorname{dim} Y_{2}-\operatorname{codim} \operatorname{Im} S=\operatorname{ind}(T)+\operatorname{ind}(S)$.

Caso geral Em geral se $S \in \mathcal{B}(X, Y)$ é semi-Fredholm com índice menor ou igual a 0 , a $\operatorname{dim}$ ker $S=m$ nos dá codim $I m S \geq m$. Escolha um espaço $m$ dimensional $Y_{1} \subset Y$ com $Y_{1} \cap \operatorname{Im} S=\{0\}$ e uma bijeção $S_{1}$ do $\operatorname{ker} S$ sobre $Y_{1}$. Seja $X=X_{1} \oplus X_{2}$ onde $X_{1}=\operatorname{ker} S$ e tome o operador de posto finito $G: X_{1} \oplus X_{2} \longrightarrow Y_{1}:\left(x_{1}, x_{2}\right) \longrightarrow S_{1} x_{1}$. O operador $S+G: X \longrightarrow Y$ é injetor, de fato, $(S+G) x=0 \Longleftrightarrow S x_{2}+S_{1} x_{1}=0$, como $\operatorname{Im} S_{1} \cap \operatorname{Im} S=\{0\}, x_{1}=x_{2}=0 \Longrightarrow x=0$. Agora, se $\operatorname{ind}(S) \geq 0$ então codim $\operatorname{Im} S=m<\infty$ e $\operatorname{dim} k e r S \geq m$. Então podemos escolher subespaços de dimensão finita $m, X_{1} \subset \operatorname{ker} S$ e $Y_{1} \subset Y$ onde $Y_{1} \cap \operatorname{Im} S=\{0\}$ de tal maneira que $X=X_{1} \oplus X_{2}$ para algum subespaço fechado $X_{2}$ e $Y=Y_{1} \oplus Y_{2}$ com $Y_{2}=\operatorname{ImS}$. Tome então $S_{1}$ bijeção qualquer de $X_{1}$ para $Y_{1}$ e defina $L: X_{1} \oplus X_{2} \longrightarrow Y: x_{1}+x_{2} \longrightarrow S_{1} x_{1}$. Por construção o operador $L$ é sobrejetor. Dados então $T$ e $S$ operadores semiFredholm com ind $(T)+i n d(S)$ definido podemos escolher $L_{i}$ tal que $T+L_{1}$ e $S+L_{2}$ são injetores ou sobrejetores com imagem fechada. Pelo que foi provado anteriormente, $\left(T+L_{1}\right)\left(S+L_{2}\right)$ é semi-Fredholm de índice $i n d\left(T+L_{1}\right)+i n d\left(S+L_{2}\right)$. Agora se $K=L_{1} S+T L_{2}+L_{1} L_{2}$ temos que

$$
T S=\left(T+L_{1}\right)\left(S+L_{2}\right)-K
$$

onde $K$ é um operador de posto finito, ou seja, $K$ é um operador compacto. Então 
pelo teorema (2.3) TS é semi-Fredholm e

$$
i n d(T S)=i n d\left(T+L_{1}\right)\left(S+L_{2}\right)=i n d\left(T+L_{1}\right)+i n d\left(S+L_{2}\right)=i n d(T)+i n d(S)
$$

como queríamos provar.

As estimativas de Schauder são tipicamente da forma

$$
\|x\|_{X} \leq C\left(\|T x\|_{Y}+|x|_{X}\right) \forall x \in X
$$

com $T \in \mathcal{B}(X, Y)$ onde $|\cdot| X$ é uma semi-norma compacta em $X$, isto é, qualquer sequência $\left\{x_{n}\right\}$ limitada na norma $\|\cdot\|_{X}$ possui subsequência de Cauchy em respeito a $|\cdot| X$. Isto facilmente implica $\operatorname{dim} \operatorname{ker} T<\infty$ e $\operatorname{Im} T$ fechada de onde podemos concluir que $T$ é semi-Fredholm com índice $<+\infty$ - tipicamente $T$ é Fredholm de índice zero.

Exemplo 4 Para um conjunto aberto conexo limitado $\Omega \subset \mathbb{R}^{n}$, seja

$$
L=\sum_{i, j=1}^{n} a_{i j}(x) \frac{\partial^{2}}{\partial x_{i} \partial x_{j}}+K: W^{2, p} \cap W_{0}^{1, p}(\Omega) \longrightarrow L^{p}(\Omega)
$$

um operador elíptico de ordem dois, onde $a_{i j}(x)=a_{j i}(x) \in \mathcal{C}^{2}, \sum_{i, j=1}^{n} a_{i j}(x) \xi_{i} \xi_{j} \geq$ $c_{0}|\xi|^{2} \forall x, \xi \in \mathbb{R}^{n}$ e para alguma constante $c_{0}>0$ e $K=b(x) \cdot \nabla+c(x)$ é um operador diferencial de ordem menor ou igual a 1 com coeficientes pelo menos contínuos. Pelo corolário 1 de [5] temos que existe uma constante $\lambda \geq 0$ tal que o operador $L+\lambda: W^{2, p} \cap W_{0}^{1, p}(\Omega) \longrightarrow$ $L^{p}(\Omega)$ é um isomorfismo. Como o operador $\lambda: W^{2, p} \cap W_{0}^{1, p}(\Omega) \longrightarrow L^{p}(\Omega)$ é um operador compacto segue pelo teorema (2.3) que $L=L+\lambda-\lambda$ é um operador Fredholm de índice zero.

\subsection{O Teorema da Transversalidade}

O teorema da Transversalidade de Thom e Abraham [1] é uma ferramenta útil em muitos ramos da Geometria e Análise. Em [11], Henry prova uma versão generalizada desse teorema visando aplicações em dimensão infinita com atenção especial para o caso de índice negativo. Vamos estudar alguns exemplos com índice negativo no capítulo de aplicações.

Antes de enunciar o teorema precisamos de algumas definições.

Dizemos que um conjunto $F$ de um espaço topológico $X$ é magro se $F$ está contido na união enumerável de conjuntos raros, ou seja, se $F$ está contido na união enumerável de conjuntos cujo fecho não possui interior. Segue da definição de conjunto magro que a união enumerável de conjuntos magros é magro. 
Dizemos que $F$ é residual se seu complementar é magro, ou seja, $F$ é a interseção enumerável de abertos densos.

Dizemos ainda que um espaço topológico $X$ é espaço de Baire se todo subconjunto residual é denso.

Seja $f$ uma aplicação $\mathcal{C}^{k}$ entre espaços de Banach. Diremos que $x$ é ponto regular de $f$ se a derivada $f^{\prime}(x)$ é sobrejetora e possui núcleo de dimensão finita. Se $x$ não é ponto regular de $f, x$ é chamado ponto crítico de $f$. Em nossas aplicações o núcleo de $f^{\prime}$ sempre será de dimensão finita, portanto, para $x$ ser ponto crítico de $f$ será necessário e suficiente $f^{\prime}(x)$ ser não sobrejetora. Um valor crítico de $f$ é a imagem por $f$ de algum ponto crítico de $f$. Todos os outros pontos do contradomínio são chamados valores regulares de $f$, incluindo todos os pontos fora da imagem de $f$.

Sejam agora $X$ um espaço topológico de Baire e $I=[0,1]$. Para qualquer subconjunto fechado ou $\sigma$-fechado $F \subset X$ e um inteiro $m \geq 0$, dizemos que a codimensão de $F$ é maior ou igual a $m$ (codim $F \geq m$ ) se o subconjunto $\left\{\phi \in \mathcal{C}\left(I^{m}, X\right) ; \phi\left(I^{m}\right) \cap F\right.$ é não vazio $\}$ é magro em $\mathcal{C}\left(I^{m}, X\right)$. Dizemos que codim $F=k$ se $k$ é o maior inteiro satisfazendo codim $F \geq m$.

Teorema 2.6 Sejam $k, m$ inteiros positivos; $X, Y$ e $Z$ variedades de Banach de classe $\mathcal{C}^{k} ; A \subset X \times Y$ um conjunto aberto; $f: A \longrightarrow Z$ uma aplicação de classe $\mathcal{C}^{k}$ e um ponto $\xi \in Z$. Assuma que para cada $(x, y) \in f^{-1}(\xi)$ :

1. $\frac{\partial f}{\partial x}(x, y): T_{x} X \longrightarrow T_{\xi} Z$ é semi-Fredholm com índice $<k$.

2. ( $\alpha) D f(x, y): T_{x} X \times T_{y} Y \longrightarrow T_{\xi} Z$ é sobrejetora

ou

( $\beta) \operatorname{dim}\left\{\frac{\operatorname{Im}(D f(x, y))}{\operatorname{Im}\left(\frac{\partial f}{\partial x}(x, y)\right)}\right\} \geq m+\operatorname{dim} \operatorname{ker}\left(\frac{\partial f}{\partial x}(x, y)\right)$.

3. $(x, y) \longrightarrow y: f^{-1}(\xi) \longrightarrow Y$ é $\sigma$-própria, ou seja, $f^{-1}(\xi)$ é a união enumerável de conjuntos $M_{j}$ tais que $(x, y) \longrightarrow y: M_{j} \longrightarrow Y$ é uma aplicação própria para cada $j \cdot\left[D a d o\left(x_{n}, y_{n}\right) \in M_{j}\right.$ tal que $\left\{y_{n}\right\}$ converge em $Y$, existe uma subsequência convergente de $\left\{\left(x_{n}, y_{n}\right)\right\}$ com limite em $M_{j}$.J

Observamos que (3) se verifica se $f^{-1}(\xi)$ é Lindelof, ou mais especificamente se $f^{-1}(\xi)$ é um espaço métrico separável, ou se $X$ e $Y$ são espaços métricos separáveis.

Seja $A_{y}=\{x \in X ;(x, y) \in A\} e$

$$
Y_{\text {crit }}=\left\{y \in Y ; \xi \text { é valor crítico de } f(\cdot, y): A_{y} \longrightarrow Z\right\} \text {. }
$$




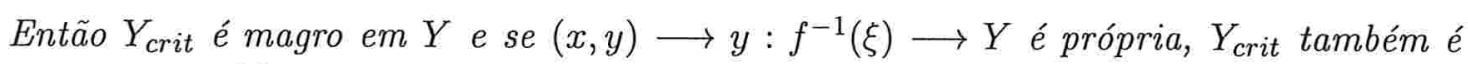
fechado. Se ind $\left(\frac{\partial f}{\partial x}(x, y)\right) \leq-m<0$ em $f^{-1}(\xi)$ então $2(\alpha)$ implica $2(\beta)$ e

$$
Y_{\text {crit }}=\left\{y \in Y ; \xi \in f\left(A_{y}, y\right)\right\}
$$

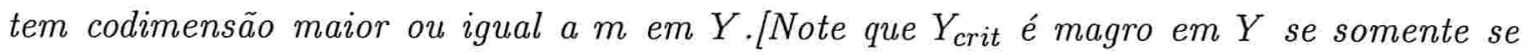
codim $Y_{\text {crit }} \geq 1$.]

A hipótese usual é a de que $\xi$ é valor regular de $f$, assim sempre temos $2(\alpha)$. Se $2(\beta)$ se verifica em algum ponto, então $\operatorname{ind}\left(\frac{\partial f}{\partial x}(x, y)\right) \leq-m$ neste ponto já que a

$$
\operatorname{codim} \operatorname{Im}\left(\frac{\partial f}{\partial x}(x, y)\right) \geq \operatorname{dim}\left\{\frac{\operatorname{Im}(D f(x, y))}{\operatorname{Im}\left(\frac{\partial f}{\partial x}(x, y)\right)}\right\} .
$$

Se o ind $\left(\frac{\partial f}{\partial x}(x, y)\right) \leq-m$ e $2(\alpha)$ se verifica, então $2(\beta)$ também se verifica como nos diz o teorema. Portanto a hipótese $2(\beta)$ é mais geral para o caso de índice negativo. 


\section{Aplicações}

Suponhamos que $\mathcal{P}$ é uma propriedade que depende de um parâmetro $x \in X$, onde $X$ é um espaço topológico de Baire. Dizemos que $\mathcal{P}$ é genérica $(\mathrm{em} x$ ) se ela vale para todo $x$ em um conjunto residual de $X$.

Em nossas aplicações (exceto a última) $X$ será a classe de regiões $\mathcal{C}^{m}$ difeomorfas a uma região fixa $\Omega_{0}$ de classe $\mathcal{C}^{m}$, ou seja,

$$
X=\left\{h\left(\Omega_{0}\right) ; h \in \operatorname{Diff}^{m}\left(\Omega_{0}\right)\right\} .
$$

Introduzimos uma topologia em $X$ definindo uma sub-base das vizinhanças de um $\Omega \in X$ por

$$
\left\{h(\Omega) ;\left\|h-i_{\Omega}\right\|_{\mathcal{C}^{m}\left(\Omega, \mathbb{R}^{n}\right)}<\epsilon, \epsilon>0 \text { suficientemente pequeno }\right\} .
$$

Michelleti prova em [15] que este é de fato um espaço topológico metrizável, portanto de Baire. De fato, provaremos nas aplicações é que a propriedade $\mathcal{P}$ vale para todo $h$ fora de um conjunto magro de $\operatorname{Diff}^{m}\left(\Omega_{0}\right)$.

Entretanto nessas aplicações, o conjunto $F$ de mergulhos excluídos será sempre definido pelas propriedades da imagem do mergulho, portanto é invariante por composição com difeomorfismos de classe $\mathcal{C}^{m}$ de $\Omega_{0}$ em $\Omega_{0}$. Isto implica (ver [11]) que o subconjunto das regiões definidas por $F$ também é magro no nosso espaço $X$. 


\subsection{Preliminares}

Consideremos o operador diferencial elíptico de segunda ordem

$$
A(\lambda)=\sum_{i, j=1}^{n} a_{i j}(x) \frac{\partial^{2}}{\partial x_{i} \partial x_{j}}+\sum_{j=1}^{n} b_{j}(x, \lambda) \frac{\partial}{\partial x_{j}}+c(x, \lambda)
$$

onde $a_{i j}(x)=a_{j i}(x) \in \mathcal{C}^{2}, b_{j}(x, \lambda), c(x, \lambda)$ são polinômios em $\lambda$ com coeficientes $\mathcal{C}^{1}$ todos assumindo valores reais quando $\lambda$ é real. Assumimos também que $\sum_{i, j=1}^{n} a_{i j}(x) \xi_{i} \xi_{j} \geq$ $c_{0}|\xi|^{2} \forall x, \xi \in \mathbb{R}^{n}$ para alguma constante $c_{0}>0$.

Impondo condições convenientes de contorno, podemos definir operadores sobre diversos espaços de funções. Aqui consideramos $A(\lambda)$ como um operador em $L^{2}(\Omega)$ com domínio $H^{2} \cap H_{0}^{1}(\Omega)$.

Dado uma região limitada $\mathcal{C}^{2}$-regular $\Omega \subset \mathbb{R}^{n}$, dizemos que $\lambda$ é autovalor de $A(\lambda)$ se existe uma solução não nula $u$ de

$$
A(\lambda) u=0 \text { em } \Omega, u=0 \text { em } \partial \Omega .
$$

$\lambda$ é autovalor simples se o núcleo de $A(\lambda)$ é unidimensional e sua imagem contida em $L^{2}(\Omega)$ não contém $\frac{d}{d \lambda}(A(\lambda)) u$ para $u \neq 0$ no núcleo.

Proposição 3.1 Seja $h \in \operatorname{Diff}^{m}(\Omega)$. A aplicação

$$
\begin{aligned}
G_{h}: H^{2} \cap H_{0}^{1}(\Omega)-\{0\} \times \mathbb{R} & \longrightarrow L^{2}(\Omega) \\
(u, \lambda) & \longrightarrow h^{*} A(\lambda) h^{*-1} u
\end{aligned}
$$

tem zero como valor regular se somente se todos os autovalores reais de

$$
h^{*} A(\lambda) h^{*-1} u=0 \text { em } \Omega, u=0 \text { sobre } \partial \Omega, u \neq 0
$$

são simples.

Prova. Considere $A^{\prime}(\lambda)=\frac{d}{d \lambda}(A(\lambda))$. A diferencial de $G_{h}$ em $(u, \lambda) \in H^{2} \cap H_{0}^{1}(\Omega)-0 \times \mathbb{R}$ é

$$
\begin{aligned}
D G_{h}(u, \lambda): H^{2} \cap H_{0}^{1}(\Omega) \times \mathbb{R} & \longrightarrow L^{2}(\Omega) \\
(\dot{u}, \dot{\lambda}) & \longrightarrow h^{*} A(\lambda) h^{*-1} \dot{u}+\dot{\lambda} h^{*} A^{\prime}(\lambda) h^{*-1} u
\end{aligned}
$$

Pelo exemplo 4 capítulo 2 temos que ind $(A(\lambda))=0$ como aplicação de $H^{2} \cap H_{0}^{1}(\Omega)$ em $L^{2}(\Omega)$, portanto ind $(A(\lambda))=1$ como aplicação de $H^{2} \cap H_{0}^{1}(\Omega) \times \mathbb{R}$ em $L^{2}(\Omega)$. Daí, como $h^{*}$ é um isomorfismo temos $\operatorname{ind}\left(h^{*} A(\lambda) h^{*-1}\right)=1$. Agora, a aplicação linear contínua 


\subsection{Simplicidade Genérica dos Autovalores do Laplaciano}

Nesta seção mostraremos que genericamente no conjunto das regiões abertas, conexas, limitadas $\mathcal{C}^{3}$-regulares $\Omega \subset \mathbb{R}^{n}$, todos os autovalores $\lambda$ de

$$
\Delta u+\lambda u=0 \text { em } \Omega, u=0 \text { sobre } \partial \Omega, u \neq 0
$$

são simples. Isto foi demonstrado originalmente por Micheletti [15] e Uhlenbeck [17].

O operador $(\Delta+\lambda)$ com domínio $H^{2} \cap H_{0}^{1}(\Omega)$ é um operador do tipo (3.1), logo as proposições da seção 3.1 podem ser aplicadas. Observe que $\lambda$ é autovalor simples de (3.5) se somente se a dimensão do espaço gerado pelas autofunções associadas a $\lambda$ é 1 . Tal afirmação segue facilmente do lema a seguir.

Lema 3.3 Consideremos o operador $(\Delta+\lambda): H^{2} \cap H_{0}^{1}(\Omega) \longrightarrow L^{2}(\Omega)$. Então temos $\operatorname{Im}(\Delta+\lambda)=\{\operatorname{ker}(\Delta+\lambda)\}^{\perp}$.

Prova. Dado $v \in H^{2} \cap H_{0}^{1}(\Omega)$ e $u \in \operatorname{ker}(\Delta+\lambda)$ temos pela segunda identidade de Green que

$$
\int_{\Omega} u(\Delta+\lambda) v=\int_{\Omega} v(\Delta+\lambda) u=0
$$

implicando que a imagem do operador $(\Delta+\lambda)$ em $H^{2} \cap H_{0}^{1}(\Omega)$ está contida no $\{u\}^{\perp}$. Vimos no exemplo 4 capítulo 2 que o operador $(\Delta+\lambda): H^{2} \cap H_{0}^{1}(\Omega) \longrightarrow L^{2}(\Omega)$ é Fredholm de índice zero, portanto a $\operatorname{dim} \operatorname{ker}(\Delta+\lambda)=\operatorname{codim}(\Delta+\lambda)$ de onde segue a afirmação do lema.

Considere a aplicação diferenciável

$$
\begin{aligned}
F: H^{2} \cap H_{0}^{1}(\Omega)-\{0\} \times \mathbb{R} \times \operatorname{Diff}^{3}(\Omega) & \longrightarrow L^{2}(\Omega) \\
(u, \lambda, h) & \longrightarrow h^{*}(\Delta+\lambda) h^{*-1} u .
\end{aligned}
$$

Segue da proposição (3.2) que estudar a simplicidade genérica dos autovalores de (3.5) é equivalente a estudar a simplicidade genérica dos autovalores de (3.3) para $A(\lambda)=(\Delta+\lambda)$.

Desta maneira, mostraremos a simplicidade genérica de todos os autovalores do probema de Dirichlet para $\Delta$ mostrando que 0 é valor regular de $G_{h}$, com $A(\lambda)=(\Delta+\lambda)$, para todo $h \in \operatorname{Diff}^{3}(\Omega)$, exceto um conjunto magro, através do teorema da Transversalidade. Portanto, nossos esforços agora se concentram em verificar as hipóteses do teorema da Trasversalidade para a aplicação diferenciável $F$.

Observe que as hipóteses (1) e (3) do teorema são satisfeitas diretamente já que o operador linear

$$
\frac{\partial F}{\partial(u, \lambda)}(u, \lambda, h): H^{2} \cap H_{0}^{1}(\Omega) \times \mathbb{R} \longrightarrow L^{2}(\Omega)
$$


$(\dot{u}, \dot{\lambda}) \longrightarrow \dot{\lambda} h^{*} A^{\prime}(\lambda) h^{*-1} u$ de $H^{2} \cap H_{0}^{1}(\Omega) \times \mathbb{R}$ em $L^{2}(\Omega)$ é compacta, e então, pelo teorema (2.3), temos que $\operatorname{ind}\left(D G_{h}(u, \lambda)\right)=1$.

Suponha que zero é valor regular de $G_{h}$, ou seja, $D G_{h}(u, \lambda)$ é sobrejetora para todo $(u, \lambda) \in G_{h}^{-1}(0)$. O ind $\left(D G_{h}(u, \lambda)\right)=1$ implica que o núcleo de $D G_{h}(u, \lambda)$ é unidimensional e igual ao subespaço gerado por $(u, 0)$. Isto implica que o núcleo de $h^{*} A(\lambda) h^{*-1}$ como aplicação de $H^{2} \cap H_{0}^{1}(\Omega)$ em $L^{2}(\Omega)$ é unidimensional. Além disso, como

$$
D G_{h}(u, \lambda)(\dot{u}, \dot{\lambda})=0 \Longleftrightarrow \dot{\lambda} h^{*} A^{\prime}(\lambda) h^{*-1} u=-h^{*} A(\lambda) h^{*-1} \dot{u}
$$

temos que $h^{*} A^{\prime}(\lambda) h^{*-1} u$ não pertence a imagem de $\left.h^{*} A(\lambda) h^{*-1}\right|_{H^{2} \cap H_{0}^{1}(\Omega)}$ implicando que $\lambda$ é autovalor simples. Logo todos os autovalores de (3.3) são simples.

Reciprocamente, suponha que todos os autovalores reais de (3.3) são simples. Então se $(u, \lambda) \in G_{h}^{-1}(0)$ temos que $\operatorname{ker}\left(h^{*} A(\lambda) h^{*-1}\right)=[u]$ e $\dot{\lambda} h^{*} A^{\prime}(\lambda) h^{*-1} u \neq-h^{*} A(\lambda) h^{*-1} \dot{u}$ para todo $\dot{u}$ e $\dot{\lambda} \neq 0$ o que implica $D G_{h}(u, \lambda)(\dot{u}, \dot{\lambda})=0 \Longleftrightarrow \dot{u}=u$ e $\dot{\lambda}=0$, ou seja, $\operatorname{dim} \operatorname{ker}\left(h^{*} A(\lambda) h^{*-1}\right)=1$.

Assim, sendo $\operatorname{ind}\left(D G_{h}(u, \lambda)\right)=1$ temos que $\operatorname{codim} \operatorname{Im}\left(D G_{h}(u, \lambda)\right)=0$ o que nos dá $D G_{h}(u, \lambda)$ sobrejetora, de onde segue o resultado.

Proposição $3.2 \lambda$ é autovalor simples de (3.3) se somente se $\lambda$ é autovalor simples de

$$
A(\lambda) v=0 \text { em } h(\Omega), v=0 \text { sobre } \partial h(\Omega), v \neq 0 .
$$

Prova. Observemos que

$$
h^{*} A(\lambda) h^{*-1} u=0 \Longleftrightarrow A(\lambda) h^{*-1} u=0
$$

pois $h^{*}$ é um isomorfismo. Então $v=h^{*-1} u \in H^{2} \cap H_{0}^{1}(h(\Omega))$ é autofunção de (3.4) associada a $\lambda$ em $h(\Omega)$ se somente se $u$ é autofunção de (3.3) associada a $\lambda$, ou seja, $\lambda$ é autovalor de (3.3) se somente se é autovalor de (3.4) em $h(\Omega)$, portanto o $\operatorname{ker}\left(h^{*} A(\lambda) h^{*-1}\right) \subset$ $H^{2} \cap H_{0}^{1}(\Omega)$ é unidimensional se somente se o $\operatorname{ker}(A(\lambda)) \subset H^{2} \cap H_{0}^{1}(h(\Omega))$ é unidimensional.

Para concluirmos a prova, basta mostrarmos que

$$
\frac{d}{d \lambda}(A(\lambda)) v \in \operatorname{Im}(A(\lambda)) \Longleftrightarrow \frac{d}{d \lambda}\left(h^{*} A(\lambda) h^{*-1}\right) u \in \operatorname{Im}\left(h^{*} A(\lambda) h^{*-1}\right) .
$$

De fato, como $\frac{d}{d \lambda}\left(h^{*} A(\lambda) h^{*-1}\right)=h^{*} \frac{d}{d \lambda}(A(\lambda)) h^{*-1}$ e $h^{*}$ é um isomorfismo temos

$$
\begin{aligned}
\frac{d}{d \lambda}(A(\lambda)) v \in \operatorname{Im}(A(\lambda)) & \Longleftrightarrow \frac{d}{d \lambda}(A(\lambda)) h^{*-1} u \in \operatorname{Im}(A(\lambda)) \\
& \Longleftrightarrow h^{*} \frac{d}{d \lambda}(A(\lambda)) h^{*-1} u \in \operatorname{Im}\left(h^{*} A(\lambda) h^{*-1}\right),
\end{aligned}
$$

de onde segue o resultado. 


$$
(\dot{u}, \dot{\lambda}) \longrightarrow h^{*}(\Delta+\lambda) h^{*-1} \dot{u}+\dot{\lambda} u
$$

é Fredholm de índice 1 e as variedades de Banach $\operatorname{Diff}^{3}(\Omega), \mathbb{R}, H^{2} \cap H_{0}^{1}(\Omega)-\{0\}$ são separáveis. Resta-nos então verificar a hipótese $2(\alpha)$ do teorema, ou seja, basta-nos mostrar que $F$ possui zero como valor regular. Faremos tal prova por absurdo.

Suponha que exista um ponto crítico $\left(u_{0}, \lambda_{0}, h_{0}\right)$ de $F$ tal que $F\left(u_{0}, \lambda_{0}, h_{0}\right)=0$. Sejam $\Omega_{0}=h_{0}(\Omega)$ e $v_{0}=h_{0}^{*-1} u_{0}$ e defina uma nova aplicação diferenciável

$$
\begin{aligned}
\tilde{F}: H^{2} \cap H_{0}^{1}\left(\Omega_{0}\right)-\{0\} \times \mathbb{R} \times \operatorname{Diff}^{3}\left(\Omega_{0}\right) & \longrightarrow L^{2}\left(\Omega_{0}\right) \\
(v, \lambda, h) & \longrightarrow h^{*}(\Delta+\lambda) h^{*-1} v .
\end{aligned}
$$

Sabendo que $\left(h \circ h_{0}\right)^{*}=h_{0}^{*} h^{*}$ temos que

$$
h_{0}^{*-1} F\left(h_{0}^{*} v, \lambda, h \circ h_{0}\right)=h_{0}^{*-1}\left(h \circ h_{0}\right)^{*}(\Delta+\lambda)\left(h \circ h_{0}\right)^{*-1} h_{0}^{*} v=\tilde{F}(v, \lambda, h) .
$$

Sabendo isto e tendo que $h_{0}^{*}$ um isomorfismo, temos que $\tilde{F}(v, \lambda, h)$, com $\tilde{F}\left(v_{0}, \lambda_{0}, i_{\Omega_{0}}\right)=0$ possui $\left(v_{0}, \lambda_{0}, i_{\Omega_{0}}\right)$ como ponto crítico se somente se $\left(u_{0}, \lambda_{0}, h_{0}\right)$ é ponto crítico de $F$ com $F\left(u_{0}, \lambda_{0}, h_{0}\right)=0$. Desta forma temos a seguinte observação:

Observação 3.1 Podemos supor sem perda de generalidade que o ponto crítico $\left(u_{0}, \lambda_{0}, h_{0}\right)$ de $F$ tal que $F\left(u_{0}, \lambda_{0}, h_{0}\right)=0$ tem $h_{0}=i_{\Omega}$, simplificando assim nossos cálculos e notação.

É importante observar também que tal observação é válida para qualquer operador diferencial $A(\lambda)$ no lugar de $(\Delta+\lambda)$ pois a forma particular do operador não foi usada no argumento acima.

Calculando então a diferencial de $F$ no ponto crítico $\left(u_{0}, \lambda_{0}, i_{\Omega}\right)$ através do teorema (1.8) obtemos pela hipótese de absurdo que

$$
\begin{aligned}
D F\left(u_{0}, \lambda_{0}, i_{\Omega}\right)(\dot{u}, \dot{\lambda}, \dot{h}) & =\left(\Delta+\lambda_{0}\right) \dot{u}+\dot{\lambda} u_{0}+\left[\dot{h} \cdot \nabla, \Delta+\lambda_{0}\right] u_{0} \\
& =\left(\Delta+\lambda_{0}\right)\left(\dot{u}-\dot{h} \cdot \nabla u_{0}\right)+\dot{\lambda} u_{0}
\end{aligned}
$$

não é sobrejetora.

Observemos que a imagem de $D F\left(u_{0}, \lambda_{0}, i_{\Omega}\right)$ em $\left\{\dot{u} \in H^{2} \cap H_{0}^{1}(\Omega), \dot{\lambda}=0, \dot{h}=0\right\}$ coincide com a imagem do operador de Fredholm $\left(\Delta+\lambda_{0}\right)$ em $H^{2} \cap H_{0}^{1}(\Omega)$ que é fechada e possui codimensão finita. Agora, como tal conjunto está contido na imagem de $D F\left(u_{0}, \lambda_{0}, i_{\Omega}\right)$, podemos concluir que a imagem de $D F\left(u_{0}, \lambda_{0}, i_{\Omega}\right)$ tem codimensão finita e portanto é fechada. Como este operador é não sobrejetor, podemos afirmar também que a 
codimensão de sua imagem é positiva. Assim sendo, como $L^{2}(\Omega)$ é um espaço de Hilbert e a imagem de $D F\left(u_{0}, \lambda_{0}, i_{\Omega}\right)$ é fechada, existe $\psi \in L^{2}(\Omega)$, não nula, ortogonal a imagem de $D F\left(u_{0}, \lambda_{0}, i_{\Omega}\right)$.

Tomando $\dot{\lambda}=\dot{h}=0$ temos $\int_{\Omega} \psi\left(\Delta+\lambda_{0}\right) \dot{u}=0 \forall \dot{u} \in H^{2} \cap H_{0}^{1}(\Omega)$, ou seja, $\psi$ é solução fraca de (3.5). Pelo fato de estarmos trabalhando com $\left(\Delta+\lambda_{0}\right)$ em $H^{2} \cap H_{0}^{1}(\Omega)$, com $\Omega$ conexa limitada $\mathcal{C}^{3}$-regular, podemos afirmar que $\psi \in H^{2} \cap H_{0}^{1}(\Omega)$ e é solução forte de (3.5). Tal afirmação segue, por exemplo, do teorema (8.2') de [2]. De fato, sendo $\Omega \in \mathcal{C}^{3}$, segue do teorema (15.2) de [6] que $u_{0}, \psi \in H^{3}(\Omega)$, então pelo teorema (11.1) de [9] $u_{0}, \psi \in \mathcal{C}^{2}(\Omega)$ de onde obtemos pelo teorema (2.28) em [8] que $u_{0}, \psi \in \mathcal{C}^{2, \alpha}(\Omega) \forall \alpha>1$, já que $u_{0}, \psi$ satisfazem (3.5).

Tomando então $\dot{h}$ em $T_{i_{\Omega}}\left(\operatorname{Diff}^{3}(\Omega)\right)=\mathcal{C}^{3}\left(\Omega, R^{n}\right)$ temos

$$
\begin{aligned}
0 & =\int_{\Omega} \psi\left(\Delta+\lambda_{0}\right) \dot{h} \cdot \nabla u_{0} \\
& =\int_{\Omega}\left\{\psi\left(\Delta+\lambda_{0}\right) \dot{h} \cdot \nabla u_{0}-\left(\Delta+\lambda_{0}\right) \psi \dot{h} \cdot \nabla u_{0}\right\} \\
& =\int_{\partial \Omega}\left\{\psi \frac{\partial\left(\dot{h} \cdot \nabla u_{0}\right)}{\partial N}-\frac{\partial \psi}{\partial N} \dot{h} \cdot \nabla u_{0}\right\} \\
& =-\int_{\partial \Omega} \dot{h} \cdot N \frac{\partial \psi}{\partial N} \frac{\partial u_{0}}{\partial N} \forall \dot{h} \in \mathcal{C}^{3}\left(\Omega, \mathbb{R}^{n}\right) .
\end{aligned}
$$

Portanto $\frac{\partial \psi}{\partial N} \frac{\partial u_{0}}{\partial N} \equiv 0$ em $\partial \Omega$ e nossa hipótese de absurdo implica na existência de $\psi \in L^{2}(\Omega)$, não nula, solução de (3.5) tal que $\frac{\partial \psi}{\partial N} \frac{\partial u_{0}}{\partial N} \equiv 0$ em $\partial \Omega$.

Para concluirmos nosso exemplo, utilizaremos o teorema de Cauchy (1.3) para equações elípticas de segunda ordem. Suponha que para algum $x_{0} \in \partial \Omega \frac{\partial u_{0}}{\partial N} \neq 0$. Então $\frac{\partial u_{0}}{\partial N} \neq 0$ numa vizinhança $V$ de $x_{0}$ em $\partial \Omega$, implicando $\frac{\partial \psi}{\partial N}=0$ nesta vizinhança. Assim temos $\frac{\partial \psi}{\partial N}=0$ e $\psi=0$ em $V$ enquanto $\left(\Delta+\lambda_{0}\right) \psi=0$ em $\Omega$. Portanto, as condições do teorema de Cauchy (1.3) estão satisfeitas resultando que $\psi \equiv 0$ em $\Omega$. Mas por hipótese $\psi$ é não nula, então temos que $\frac{\partial u_{0}}{\partial N}=0 \mathrm{em} \partial \Omega$. Podemos então repetir o argumento anterior para $u_{0}$ e obtermos que $u_{0} \equiv 0$ em $\Omega$, mas por hipótese $u_{0} \in H^{2} \cap H_{0}^{1}(\Omega)-\{0\}$, contradição. Logo podemos concluir que 0 é valor regular de $F$, como queríamos mostrar. 


\subsection{Uma Propriedade Genérica para as Autofunções do La- placiano}

No exemplo anterior trabalhamos com uma condição sobre os autovalores do problema de Dirichlet. Neste exemplo trabalharemos com uma condição sobre as autofunções deste problema, mostrando que, genericamente no conjunto das regiões $\Omega \subset \mathbb{R}^{n}$ abertas, conexas, limitadas, $\mathcal{C}^{3}$-regulares, as autofunções normalizadas $u$ de (3.5) satisfazem $\int_{\Omega} g(u) \neq 0$, onde $g: \mathbb{R} \longrightarrow \mathbb{R}$ é uma função de classe $\mathcal{C}^{3}$ cujas raízes formam um conjunto discreto.

Suponha então $g$ uma função de classe $\mathcal{C}^{3}$ como acima e $\Omega \subset \mathbb{R}^{n}$ uma região aberta, conexa, limitada, $\mathcal{C}^{3}$-regular. Através do teorema da Transversalidade provaremos que o subconjunto

$B=\left\{h \in \operatorname{Diff}^{3}(\Omega) ; \int_{h(\Omega)} g(u)=0\right.$ para alguma autofunção normalizada $u$ de $(3.5)$ em $\left.h(\Omega)\right\}$ é magro em $\operatorname{Diff}^{3}(\Omega)$ de onde segue o resultado desejado.

Inicialmente vamos mostrar que as autofunções associadas a autovalores simples menores ou iguais a algum $N \in \mathbb{N}$ satisfazem genericamente $\int_{\Omega} g(u) \neq 0$, depois obteremos o resultado desejado para todas as autofunções de (3.5) através de um argumento simples.

Precisamos antes dos seguintes lemas.

Lema 3.4 Dado $h_{0} \in \operatorname{Diff}^{2}(\Omega)$ existe uma vizinhança $V_{0}$ de $h_{0}$ em $\operatorname{Diff}^{2}(\Omega)$ tal que para todo $h \in V_{0}$ temos

$$
\left\|\left(h^{*} \Delta h^{*-1}-h_{0}^{*} \Delta h_{0}^{*-1}\right) u\right\|_{L^{2}(\Omega)} \leq \epsilon(h)\|u\|_{H^{2} \cap H_{0}^{1}(\Omega)}
$$

onde $\epsilon(h)$ tende a zero quando $h \longrightarrow h_{0}$ em $\mathcal{C}^{2}\left(\Omega, \mathbb{R}^{n}\right)$.

Prova. Basta considerarmos o caso $h_{0}=i_{\Omega}$. Da seção 1.2 temos

$$
\begin{aligned}
h^{*} \frac{\partial}{\partial y_{i}} h^{*-1} u(x) & =\frac{\partial}{\partial y_{i}}\left(u \circ h^{-1}\right)(h(x)) \\
& =\sum_{j=1}^{n} \frac{\partial u}{\partial x_{j}}(x)\left(h_{x}^{-1}\right)_{j i}(x) \\
& =\sum_{j=1}^{n} b_{i j}(x) \frac{\partial u}{\partial x_{j}}(x)
\end{aligned}
$$

onde $b_{i j}(x)=\left(h_{x}^{-1}\right)_{j i}(x)$, ou seja, $b_{i j}(x)$ é a $i, j$-ésima entrada da transposta da matriz Jacobiana inversa de $h_{x}=\left(\frac{\partial h_{i}}{\partial x_{j}}\right)_{i, j=1}^{n}$. Portanto

$$
h^{*} \frac{\partial^{2}}{\partial y_{i}^{2}} h^{*-1} u(x)=\sum_{k=1}^{n} b_{i k}(x) \frac{\partial}{\partial x_{k}}(x)\left(\sum_{j=1}^{n} b_{i j}(x) \frac{\partial u}{\partial x_{j}}\right)(x)
$$




$$
\begin{aligned}
& =\sum_{k=1}^{n} b_{i k}(x) \sum_{j=i}^{n}\left[\left(\frac{\partial}{\partial x_{k}} b_{i j}\right)(x) \frac{\partial u}{\partial x_{j}}+b_{i j}(x) \frac{\partial^{2} u}{\partial x_{k} \partial x_{j}}(x)\right] \\
& \left.=\sum_{j, k=1}^{n} b_{i k}(x) b_{i j}(x)\left(\frac{\partial^{2} u}{\partial x_{k} \partial x_{j}}\right)(x)+\sum_{j, k=1}^{n} b_{i k}(x)\left(\frac{\partial}{\partial x_{k}} b_{i j}\right)(x)\right)(x) \frac{\partial u}{\partial x_{j}}(x) \\
& =\left(\frac{\partial^{2}}{\partial x_{i}^{2}}(u)\right)(x)+L_{i}(u)(x)
\end{aligned}
$$

onde

$$
\begin{aligned}
L_{i}(u)(x)= & \left(b_{i i}^{2}(x)-1\right)\left(\frac{\partial^{2}}{\partial x_{i}^{2}}(u)\right)(x)+\sum_{j, k=1}^{n}\left(1-\delta_{i, j, k}\right) b_{i, k}(x) b_{i, j}(x)\left(\frac{\partial^{2} u}{\partial x_{k} \partial x_{j}}\right)(x)+ \\
& \left.+\sum_{j, k=1}^{n} b_{i, k}(x)\left(\frac{\partial}{\partial x_{k}} b_{i j}\right)(x)\right)(x) \frac{\partial u}{\partial x_{j}}(x) .
\end{aligned}
$$

Então

$$
\left(h^{*} \Delta h^{*-1}(u)\right)=\Delta u+L u
$$

com

$$
L u=\sum_{i=1}^{n} L_{i} u .
$$

Já que $b_{j, k} \longrightarrow \delta_{j, k}$ em $\mathcal{C}^{2}\left(\Omega, \mathbb{R}^{n}\right)$ quando $h \longrightarrow i_{\Omega}$ em $\mathcal{C}^{2}\left(\Omega, \mathbb{R}^{n}\right)$ temos que os coeficientes de $L$ tendem a 0 uniformemente quando $h \longrightarrow i_{\Omega}$ em $\mathcal{C}^{2}\left(\Omega, \mathbb{R}^{n}\right)$. Então segue que

$$
\|L u\|_{L^{2}(\Omega)} \leq \epsilon(h)\|u\|_{H^{2} \cap H_{0}^{1}(\Omega)}
$$

onde $\epsilon(h)$ tende a zero quando $h \longrightarrow i_{\Omega}$ em $\mathcal{C}^{2}\left(\Omega, \mathbb{R}^{n}\right)$.

Seja

$$
\begin{aligned}
D_{N}= & \left\{h \in \operatorname{Diff}^{3}(\Omega) ; N \text { não é autovalor de }(3.5) \text { em } h(\Omega)\right. \\
& \text { e todos os autovalores em }(0, N) \text { de }(3.5) \text { em } h(\Omega) \text { são simples }\} .
\end{aligned}
$$

Lema 3.5 $D_{N}$ é um subconjunto aberto e denso de $\operatorname{Diff}^{3}(\Omega)$.

Prova. Defina

$$
D=\left\{h \in \operatorname{Diff}^{3}(\Omega) ; \text { todos os autovalores de (3.5) em } h(\Omega) \text { são simples }\right\}
$$

e

$$
\tilde{D_{N}}=\left\{h \in \operatorname{Diff}^{3}(\Omega) ; \text { todos os autovalores em }(0, N) \text { de }(3.5) \text { em } h(\Omega) \text { são simples }\right\} .
$$

Vamos mostrar primeiro que $D_{N}$ é aberto. Sejam $h_{0} \in D_{N}$ e $\lambda_{1}, \ldots, \lambda_{k}$ autovalores de $\Delta$ em $h_{0}(\Omega)$ menores que $N$. Considere um círculo $\gamma$ de raio $N$. 
O lema anterior e os teoremas 2.14 e 3.16 de [14] nos garantem que existe uma vizinhança $V_{0}$ de $h_{0}$ tal que a dimensão de auto-espaço associado aos autovalores de norma menor que $N$ é $k$ para todo operador $h^{*} \Delta h^{*-1}$ com $h \in V_{0}$. Pelo exemplo 2 capítulo 1 temos que cada autovalor simples de $h_{0}{ }^{*} \Delta h_{0}{ }^{*-1}$ depende continuamente de $h$ numa vizinhança $\mathcal{C}^{2}$ de $h_{0}$. Então, para cada $1 \leq i \leq k$ existe $V_{i} \subset \operatorname{Diff}^{3}(\Omega)$ vizinhança de $h_{0}$ e $\Lambda_{i}: V_{i} \longrightarrow(0, N)$ contínuas tal que $\Lambda_{i}(h)$ é autovalor simples de $h^{*} \Delta h^{*-1}$ para todo $h \in V_{i} \operatorname{com} \Lambda_{i}\left(h_{0}\right)=\lambda_{i}$ e os conjuntos $\Lambda_{i}\left(V_{i}\right)$ são dois a dois disjuntos. Defina então $V=\bigcap_{i=0}^{k} V_{i}$, vizinhança de $h_{0}$ em $\operatorname{Diff}^{3}(\Omega)$. Observe que $\forall h \in V, h^{*} \Delta h^{*-1}$ possui $k$ autovalores menores que $N$ todos simples. Portanto $D_{N}$ é aberto.

No exemplo anterior vimos que o complementar de $D$ é magro, logo temos que $D$ é denso em $\operatorname{Diff}^{3}(\Omega)$. Como $\tilde{D_{N}} \supset D$, podemos concluir que $\tilde{D_{N}}$ é denso em $\operatorname{Diff}^{3}(\Omega)$. Portanto, para provar que $D_{N}$ é denso basta mostrar que se $N$ é autovalor de (3.5) em uma região $\Omega$, então existe $h$ próximo de $i_{\Omega}$ tal que isto não se verifica em $h(\Omega)$. De fato, basta tomar $h(x)=(1+\epsilon) x$. Um cálculo simples mostra que cada autovalor $\lambda$ de $\Delta$ em $\Omega$ se transforma em um autovalor $\frac{\lambda}{(1+\epsilon)^{2}} \operatorname{em~} h(\Omega)$.

Seja $\Omega \subset \mathbb{R}^{n}$ uma região $\mathcal{C}^{3}$-regular, aberta, conexa, limitada e considere a aplicação diferenciável

$$
\begin{aligned}
F: H^{2} \cap H_{0}^{1}(\Omega)-\{0\} \times(0, N) \times D_{N} & \longrightarrow L^{2}(\Omega) \times \mathbb{R} \times \mathbb{R} \\
(u, \lambda, h) & \longrightarrow\left(h^{*}(\Delta+\lambda) h^{*-1} u, \int_{\Omega} u^{2} \operatorname{det} h^{\prime}, \int_{\Omega} g(u) \operatorname{det} h^{\prime}\right) .
\end{aligned}
$$

Através do teorema da transversalidade mostraremos que o subconjunto

$$
B_{N}=\left\{h \in D_{N} ;(0,1,0) \in F\left(H^{2} \cap H_{0}^{1}(\Omega)-\{0\},(0, N), h\right)\right\}
$$

é magro em $D_{N}$ de onde segue o resultado.

Afirmamos inicialmente que o operador contínuo $\frac{\partial F}{\partial(u, \lambda)}(u, \lambda, h)$ de $H^{2} \cap H_{0}^{1}(\Omega) \times \mathbb{R}$ em $L^{2}(\Omega) \times \mathbb{R} \times \mathbb{R}$ é Fredholm com ind $\left(\frac{\partial F}{\partial(u, \lambda)}(u, \lambda, h)\right) \leq-1 \forall(u, \lambda, h) \in F^{-1}(0,1,0)$.

Seja $(u, \lambda, h) \in F^{-1}(0,1,0)$. Podemos supor sem perda de generalidade $h=i_{\Omega}$ como na observação (3.1). De fato, sempre que $F(u, \lambda, h)=(0,1,0), v=h^{*-1} u$ satisfaz

$$
\Delta v+\lambda v=0 \text { em } h(\Omega), v=0 \operatorname{em} \partial h(\Omega) \text { e } \int_{h(\Omega)} v^{2}=1 .
$$

Calculando as derivadas temos

$$
\begin{aligned}
D F_{1}\left(u, \lambda, i_{\Omega}\right)(\dot{u}, \dot{\lambda}, \dot{h}) & =(\Delta+\lambda)(\dot{u}-\dot{h} \cdot \nabla u)+\dot{\lambda} u \\
D F_{2}\left(u, \lambda, i_{\Omega}\right)(\dot{u}, \dot{\lambda}, \dot{h}) & =\int_{\Omega} 2 u \dot{u}+\int_{\partial \Omega} u^{2}(\dot{h} \cdot N) \\
& =\int_{\Omega} 2 u \dot{u}
\end{aligned}
$$




$$
\begin{aligned}
D F_{3}\left(u, \lambda, i_{\Omega}\right)(\dot{u}, \dot{\lambda}, \dot{h}) & =\int_{\Omega} g^{\prime}(u) \dot{u}+\int_{\partial \Omega} g(u)(\dot{h} \cdot N) \\
& =\int_{\Omega} g^{\prime}(u) \dot{u}+g(0) \int_{\partial \Omega}(\dot{h} \cdot N)
\end{aligned}
$$

onde $N$ é o vetor normal unitário a $\partial \Omega$. Logo

$$
\begin{aligned}
D F\left(u, \lambda, i_{\Omega}\right)(\dot{u}, \dot{\lambda}, \dot{h}) & =\left(D F_{1}\left(u, \lambda, i_{\Omega}\right), D F_{2}\left(u, \lambda, i_{\Omega}\right), D F_{3}\left(u, \lambda, i_{\Omega}\right)\right)(\dot{u}, \dot{\lambda}, \dot{h}) \\
& =\left((\Delta+\lambda)(\dot{u}-\dot{h} \cdot \nabla u)+\dot{\lambda} u, \int_{\Omega} 2 u \dot{u}, \int_{\Omega} g^{\prime}(u) \dot{u}+g(0) \int_{\partial \Omega}(\dot{h} \cdot N)\right)
\end{aligned}
$$

e

$$
\begin{aligned}
\frac{\partial F}{\partial(u, \lambda)}\left(u, \lambda, i_{\Omega}\right)(\dot{u}, \dot{\lambda}) & =\left(\frac{\partial F_{1}}{\partial(u, \lambda)}\left(u, \lambda, i_{\Omega}\right), \frac{\partial F_{2}}{\partial(u, \lambda)}\left(u, \lambda, i_{\Omega}\right), \frac{\partial F_{3}}{\partial(u, \lambda)}\left(u, \lambda, i_{\Omega}\right)\right)(\dot{u}, \dot{\lambda}) \\
& =\left((\Delta+\lambda) \dot{u}+\dot{\lambda} u, \int_{\Omega} 2 u \dot{u}, \int_{\Omega} g^{\prime}(u) \dot{u}\right) .
\end{aligned}
$$

Claramente $\frac{\partial F}{\partial(u, \lambda)}\left(u, \lambda, i_{\Omega}\right)$ é Fredholm pois $\frac{\partial F_{1}}{\partial(u, \lambda)}\left(u, \lambda, i_{\Omega}\right)$ é Fredholm e os operadores $F_{2}$, $F_{3}$ tem imagem em espaços de dimensão finita. Observe agora que a aplicação

$$
\left(\frac{\partial F_{1}}{\partial(u, \lambda)}\left(u, \lambda, i_{\Omega}\right), \frac{\partial F_{2}}{\partial(u, \lambda)}\left(u, \lambda, i_{\Omega}\right)\right): H^{2} \cap H_{0}^{1}(\Omega) \times \mathbb{R} \longrightarrow L^{2}(\Omega) \times \mathbb{R}
$$

é sobrejetora. De fato, dado $(f, x) \in L^{2}(\Omega) \times \mathbb{R}$ definidas por seja $(v, \xi) \in H^{2} \cap H_{0}^{1}(\Omega) \times \mathbb{R}$

$$
v=v_{0}+\frac{x u}{2} \text { e } \xi=\int_{\Omega} u f
$$

onde $v_{0} \in H^{2} \cap H_{0}^{1}(\Omega)$ satisfaz

$$
(\Delta+\lambda) v_{0}=f-\xi u \text { e } v_{0} \perp u
$$

Note que existência de $v_{0}$ é garantida pelo lema (3.3) pois $(f-\xi u) \perp u$. Então temos

$$
\begin{gathered}
\frac{\partial F_{1}}{\partial(u, \lambda)}\left(u, \lambda, i_{\Omega}\right)(\dot{u}, \dot{\lambda})=(\Delta+\lambda) v+\xi u=f-\xi u+\frac{x}{2}(\Delta+\lambda) u+\xi u=f \\
\frac{\partial F_{2}}{\partial(u, \lambda)}\left(u, \lambda, i_{\Omega}\right)(\dot{u}, \dot{\lambda})=\int_{\Omega} 2 u\left(v_{0}+\frac{x}{2} u\right)=x \int_{\Omega} u^{2}=x .
\end{gathered}
$$

Observe também que $\left(\frac{\partial F_{1}}{\partial(u, \lambda)}\left(u, \lambda, i_{\Omega}\right), \frac{\partial F_{2}}{\partial(u, \lambda)}\left(u, \lambda, i_{\Omega}\right)\right)$ é injetora, de fato, $\left(\frac{\partial F_{1}}{\partial(u, \lambda)}\left(u, \lambda, i_{\Omega}\right), \frac{\partial F_{2}}{\partial(u, \lambda)}\left(u, \lambda, i_{\Omega}\right)\right)(v, \xi)=(0,0) \quad \Longleftrightarrow \quad(\Delta+\lambda) v+\xi u=0$ e $\int_{\Omega} 2 u v=0$. Agora $(\Delta+\lambda) v+\xi u=0 \Longrightarrow u(\Delta+\lambda) v+\xi u^{2}=0$ o que implica $-\int_{\Omega} u(\Delta+\lambda) v=\xi \Longleftrightarrow$ $\xi=0$. Daí $(\Delta+\lambda) v=0 \operatorname{com} \int_{\Omega} 2 u v=0$, ou seja, $u \perp v$ e $(\Delta+\lambda) v=0$. Portanto, como 
$\lambda$ é autovalor simples associado a autofunção $u$, temos que $v \equiv 0$. Agora, como (3.6) é um operador contínuo, sobrejetor com domínio $H^{2} \cap H_{0}^{1}(\Omega)$ temos pelo teorema do Gráfico Fechado que a sua inversa é contínua em $L^{2}(\Omega)$. Portanto (3.6) é um isomorfismo.

Sendo a aplicação (3.6) um isomorfismo, podemos concluir que $\frac{\partial F}{\partial(u, \lambda)}\left(u, \lambda, i_{\Omega}\right)$ não é sobrejetora. Mais ainda, como o núcleo de tal aplicação é o subespaço nulo temos que o $\operatorname{ind}\left(\frac{\partial F}{\partial(u, \lambda)}\left(u, \lambda, i_{\Omega}\right)\right) \leq-1$. Desta maneira podemos afirmar que $\forall(u, \lambda, h) \in F^{-1}(0,1,0)$ o ind $\left(\frac{\partial F}{\partial(u, \lambda)}(u, \lambda, h)\right) \leq-1$, como queríamos verificar.

Agora vamos verificar a sobrejetividade da diferencial de $F \forall(u, \lambda, h) \in F^{-1}(0,1,0)$. Antes de mais nada observamos que basta verificar tal afirmação para $h=i_{\Omega}$ como já foi justificado anteriormente.

Seja $(f, x, y) \in L^{2}(\Omega) \times \mathbb{R} \times \mathbb{R}$. Temos que determinar $(\dot{u}, \dot{\lambda}, \dot{h})$ tal que $D F\left(u, \lambda, i_{\Omega}\right)(\dot{u}, \dot{\lambda}, \dot{h})=$ $(f, x, y)$, ou seja, devemos resolver o sistema

$$
\begin{gathered}
(\Delta+\lambda)(\dot{u}-\dot{h} \cdot \nabla u)+\dot{\lambda} u=f \\
\int_{\Omega} 2 u \dot{u}=x \\
\int_{\Omega}\left\{g^{\prime}(u) \dot{u}+g(0) \operatorname{div}(\dot{h})\right\}=y
\end{gathered}
$$

para $(\dot{u}, \dot{\lambda}, \dot{h}) \in H^{2} \cap H_{0}^{1}(\Omega) \times \mathbb{R} \times \mathcal{C}^{3}\left(\Omega, \mathbb{R}^{n}\right)$. Observemos que $\int_{\partial \Omega}(\dot{h} \cdot N)=\int_{\Omega} \operatorname{div}(\dot{h})$. Suponha $\dot{h}$ variando em $\mathcal{C}_{0}^{3}\left(\Omega, \mathbb{R}^{n}\right)$, isto é $\dot{h}$ em $\mathcal{C}^{3}\left(\Omega, \mathbb{R}^{n}\right)$ e suporte compacto. Multiplicando (3.7) por $u$ e integrando dos dois lados temos que

$$
\int_{\Omega} u(\Delta+\lambda)(\dot{u}-\dot{h} \cdot \nabla u)+\dot{\lambda} u^{2}=\int_{\Omega} u f \Longleftrightarrow \dot{\lambda}=\int_{\Omega} u f
$$

já que $\dot{u}-\dot{h} \cdot \nabla u \in H^{2} \cap H_{0}^{1}(\Omega)$. Fixemos $\dot{\lambda}=\int_{\Omega} u f$. Então como $f-\dot{\lambda} u \perp u$, pelo lema (2.3) existe $w \in H^{2} \cap H_{0}^{1}(\Omega), w \perp u$ tal que $(\Delta+\lambda)(w+\alpha u)=f-\dot{\lambda} u$. Tomemos $\dot{u}=w+\alpha u+\dot{h} \cdot \nabla u$ para $\alpha \in \mathbb{R}$ e $\dot{\lambda}=\int_{\Omega} u f$. Observe que $\dot{u} \in H^{2} \cap H_{0}^{1}(\Omega)$. Então para todo $\dot{h}$ em $\mathcal{C}_{0}^{3}\left(\Omega, \mathbb{R}^{n}\right)$ e $\alpha \in \mathbb{R}$, temos que $\dot{u}$ e $\dot{\lambda}$ satisfazem (3.7).

Se tomarmos $\alpha=\frac{x}{2}-\int_{\Omega} u(\dot{h} \cdot \nabla u)$ temos que $\dot{u}$ também satisfaz (3.8), de fato,

$$
\begin{aligned}
\int_{\Omega} 2 u \dot{u} & =\int_{\Omega} 2 u\left[w+\left(\frac{x}{2}-\int_{\Omega} u(\dot{h} \cdot \nabla u)\right) u+\dot{h} \cdot \nabla u\right] \\
& =\int_{\Omega}\left\{2 u^{2} \frac{x}{2}-\left(\int_{\Omega} u(\dot{h} \cdot \nabla u)\right) 2 u^{2}+2 u(\dot{h} \cdot \nabla u)\right\} \\
& =x .
\end{aligned}
$$

Agora observemos que

$$
\int_{\Omega} u(\dot{h} \cdot \nabla u)=\frac{1}{2} \int_{\Omega}\left(\dot{h} \cdot \nabla u^{2}\right)
$$




$$
\begin{aligned}
& =\frac{1}{2}\left(\int_{\partial \Omega} u^{2}(\dot{h} \cdot N)-\int_{\Omega} u^{2} \operatorname{div}(\dot{h})\right) \\
& =-\frac{1}{2} \int_{\Omega} u^{2} \operatorname{div}(\dot{h})
\end{aligned}
$$

onde div é o divergente. Logo $\alpha=\frac{x}{2}+\frac{1}{2} \int_{\Omega} u^{2} \operatorname{div}(\dot{h})$. Substituindo $\dot{u}$ em (3.9) encontramos a equação:

$$
\begin{aligned}
y & =\int_{\Omega}\left\{g^{\prime}(u)\left[w+\left(\frac{x}{2}+\frac{1}{2} \int_{\Omega} u^{2} \operatorname{div}(\dot{h})\right) u+\dot{h} \cdot \nabla u\right]+g(0) \operatorname{div}(\dot{h})\right\} \\
& =\int_{\Omega}\left\{g^{\prime}(u)\left[w+\left(\frac{x}{2}+\frac{1}{2} \int_{\Omega} u^{2} \operatorname{div}(\dot{h})\right) u+\dot{h} \cdot \nabla u\right]\right\}+g(0) \int_{\partial \Omega} \dot{h} \cdot N \\
& =\int_{\Omega}\left\{g^{\prime}(u)\left[w+\left(\frac{x}{2}+\frac{1}{2} \int_{\Omega} u^{2} \operatorname{div}(\dot{h})\right) u+\dot{h} \cdot \nabla u\right]\right\}
\end{aligned}
$$

que é equivalente à equação

$$
y-\int_{\Omega}\left\{g^{\prime}(u)\left(w+\frac{x u}{2}\right)\right\}=\int_{\Omega}\left\{\left[\left(\int_{\Omega} \frac{u}{2} g^{\prime}(u)\right) u^{2}-g(u)\right] \operatorname{div}(\dot{h})\right\}
$$

já que $\int_{\Omega} g^{\prime}(u)(\dot{h} \cdot \nabla u)=-\int_{\Omega} g(u) \operatorname{div}(\dot{h})$. Observe que $w, x, u, g(u)$ e $g^{\prime}(u)$ estão determinados e o lado direito da equação (3.10) é linear em $\dot{h}$, portanto podemos definir um funcional linear contínuo em $\mathcal{C}_{0}^{3}\left(\Omega, \mathbb{R}^{n}\right)$

$$
\Phi: \dot{h} \rightarrow \int_{\Omega}\left\{\left[\left(\int_{\Omega} \frac{u}{2} g^{\prime}(u)\right) u^{2}-g(u)\right] \operatorname{div}(\dot{h})\right\} .
$$

Se mostrarmos que $\Phi$ é não nulo em $\mathcal{C}_{0}^{3}\left(\Omega, \mathbb{R}^{n}\right)$, a equação será resolvida para algum $\dot{h} \epsilon$ $\mathcal{C}_{0}^{3}\left(\Omega, \mathbb{R}^{n}\right)$ e portanto $D F\left(u, \lambda, i_{\Omega}\right)$ será sobrejetora como queremos mostrar. Assim, basta mostrar que existe $\dot{h} \in \mathcal{C}_{0}^{3}\left(\Omega, \mathbb{R}^{n}\right)$ tal que $\Phi(\dot{h}) \neq 0$.

Para isto observemos que $\Phi$ se estende a um funcional linear contínuo $\tilde{\Phi}$ em $\mathcal{C}^{3}\left(\Omega, \mathbb{R}^{n}\right)$

$$
\tilde{\Phi}: \dot{h} \longrightarrow \int_{\Omega}\left\{\left[\left(\int_{\Omega} \frac{u}{2} g^{\prime}(u)\right) u^{2}-g(u)\right] d i v(\dot{h})\right\} .
$$

Observemos que dado qualquer função $v \in \mathcal{C}^{3}(\Omega)$ existe $\dot{h} \in \mathcal{C}^{3}\left(\Omega, \mathbb{R}^{n}\right)$ tal que $\operatorname{div}(\dot{h})=v$, de fato, tome $\dot{h}=\left(\dot{h_{1}}, \ldots, \dot{h_{n}}\right)$ com $\dot{h_{1}}\left(x_{1}, x_{2}, \ldots, x_{n}\right)=\int_{x_{0}}^{x_{1}} v\left(\xi, x_{2}, \ldots, x_{n}\right) d \xi$ e $\dot{h_{i}} \equiv 0$ para $2 \leq i \leq n, \operatorname{div}(\dot{h})=v$. Sendo assim, para que $\tilde{\Phi}$ não seja identicamente nulo basta mostrarmos que $\left(\int_{\Omega} \frac{u}{2} g^{\prime}(u)\right) u^{2}-g(u)$ não é a função nula, pois se $\left(\int_{\Omega} \frac{u}{2} g^{\prime}(u)\right) u^{2}-g(u) \neq 0$ podemos tomar $\dot{h} \in \mathcal{C}^{3}\left(\Omega, \mathbb{R}^{n}\right)$ tal que o $\operatorname{div}(\dot{h})=\left(\int_{\Omega} \frac{u}{2} g^{\prime}(u)\right) u^{2}-g(u)$ o que nos dá $\tilde{\Phi}(\dot{h})=\int_{\Omega}\left\{\left[\left(\int_{\Omega} \frac{u}{2} g^{\prime}(u)\right) u^{2}-g(u)\right]^{2}\right\}>0$. Agora

$$
\left(\int_{\Omega} \frac{u}{2} g^{\prime}(u)\right) u^{2}-g(u) \equiv 0 \Longleftrightarrow g(u)=\left(\int_{\Omega} \frac{u}{2} g^{\prime}(u)\right) u^{2} .
$$


Observemos agora que se $g(u)=\left(\int_{\Omega} \frac{u}{2} g^{\prime}(u)\right) u^{2}$ temos que

$$
\begin{aligned}
0 & =\int_{\Omega} g(u) \\
& =\int_{\Omega}\left(\int_{\Omega} \frac{u}{2} g^{\prime}(u)\right) u^{2} \\
& =\int_{\Omega} \frac{u}{2} g^{\prime}(u)
\end{aligned}
$$

o que implica $g(u) \equiv 0$. Mas $u \in H^{2} \cap H_{0}^{1}(\Omega)-\{0\}$ e $g$ por hipótese é uma função de classe $\mathcal{C}^{3}$ cujas raízes formam um conjunto discreto, portanto $g(u) \neq 0$ e temos que $\tilde{\Phi}$ é não nulo de onde segue imediatamente que $\Phi$ não é identicamente nulo.

Portanto, até aqui verificamos as hipóteses (1) e (2) do teorema da Transversalidade. A hipótese (3) é diretamente verificada pois $H^{2} \cap H_{0}^{1}(\Omega)-\{0\},(0, N)$ e $D_{N}$ são variedades de Banach separáveis. Logo, podemos concluir pelo teorema da Transversalidade que para cada $N \in \mathbb{N}$ temos $B_{N}$ magro em $D_{N}$.

Vamos agora mostrar que $B$ é magro em $\operatorname{Diff}^{3}(\Omega)$. Para isso, observamos primeiro que $(0,1,0) \in F\left(H^{2} \cap H_{0}^{1}(\Omega)-\{0\},(0, N), h\right)$ para $h \in D_{N}$ se somente se

$$
\Delta u+\lambda u=0 \operatorname{em} h(\Omega), \int_{h(\Omega)} u^{2}=1 \text { e } \int_{h(\Omega)} g(u)=0
$$

para algum $\lambda \in \mathbb{R}$ autovalor simples e algum $u \in H^{2} \cap H_{0}^{1}(h(\Omega))-\{0\}$. Observe que tal resultado segue facilmente da proposição (3.2). Daí podemos dizer que $B_{N}$ é o conjunto dos $h \in D_{N}$ tal que $\int_{h(\Omega)} g(u)=0$ para alguma $u$ autofunção de (3.5) em $h(\Omega)$ associada a algum autovalor simples $\lambda<N$ satisfazendo $\int_{h(\Omega)} u^{2}=1$. Assim, temos diretamente que

$$
B \subset\left\{\cup_{N \in \mathrm{N}} B_{N}\right\} \cup\left\{\operatorname{Diff}^{3}(\Omega)-D\right\}
$$

Como a união enumerável de conjuntos magros é magro, temos que $B$ está contido num subconjunto magro de $\operatorname{Diff}^{3}(\Omega)$, ou seja, $B$ é magro em $\operatorname{Diff}^{3}(\Omega)$. Assim obtemos o resultado desejado para toda $g$ de classe $\mathcal{C}^{3}$ cujas raízes formam um conjunto discreto. 


\subsection{Simplicidade Genérica dos Autovalores Reais}

Estudaremos agora a simplicidade genérica dos autovalores reais de (3.1).

Seja $A_{\Omega}(\lambda)$ o operador $A(\lambda)$ definido de $H^{2} \cap H_{0}^{1}(\Omega)$ em $L^{2}(\Omega)$ considerados com a estrutura de espaço vetorial sobre $\mathbb{R}$ e considere o problema

$$
A_{\Omega}(\lambda) u=0 \text { em } \Omega, u=0 \text { em } \partial \Omega .
$$

Suponha $\Omega \subset \mathbb{R}^{n}$ uma região conexa, limitada, $\mathcal{C}^{3}$-regular e considere a aplicação diferenciável

$$
\begin{aligned}
F: H^{2} \cap H_{0}^{1}(\Omega)-\{0\} \times \mathbb{R} \times \operatorname{Diff}^{3}(\Omega) & \longrightarrow L^{2}(\Omega) \\
(u, \lambda, h) & \longrightarrow h^{*} A_{h(\Omega)}(\lambda) h^{*-1} u
\end{aligned}
$$

O objetivo desta seção é mostrar que, genericamente no conjunto das regiões $\Omega \subset \mathbb{R}^{n}$ abertas, conexas, limitadas $\mathcal{C}^{3}$-regulares todos os autovalores reais de (3.11) são simples. É importante observar que nossas hipóteses permitem o caso em que $A_{\Omega}(\lambda)$ não depende de $\lambda$. Neste caso, $A_{\Omega}^{\prime}(\lambda) \equiv 0$ e como 0 pertence a qualquer subspaço linear, não podem existir autovalores simples para (3.11). Sendo assim, provaremos que $A_{\Omega}(\lambda)$ é genericamente um isomorfismo se não depende de $\lambda$.

A proposição (3.2) nos garante que estudar a simplicidade genérica dos autovalores de (3.11) em $h(\Omega)$ é equivalente a estudar a simplicidade genérica dos autovalores de (3.3) para $A(\lambda)=A_{\Omega}(\lambda)$. Desta maneira, mostraremos a simplicidade genérica de todos os autovalores do probema de Dirichlet para $A_{\Omega}(\lambda)$ mostrando através do teorema da Transversalidade que genericamente em $\operatorname{Diff}^{3}(\Omega)$, 0 é valor regular da aplicação $G_{h}$ definida na proposição (3.1) supondo neste caso que $A(\lambda)=A_{\Omega}(\lambda)$. Portanto, nossos esforços agora se concentram em verificar as hipóteses do teorema da Trasversalidade para a aplicação diferenciável $F$.

Observe que as hipóteses (1) e (3) do teorema são verificadas diretamente pois para $h$ fixo a aplicação $(u, \lambda) \longrightarrow F(u, \lambda, h)$ é Fredholm de índice 1 e todos os espaços envolvidos são separáveis. Assim precisamos apenas mostrar que zero é valor regular de $F$.

Suponha por absurdo que $F$ tenha um ponto crítico $(u, \lambda, h) \operatorname{com} F(u, \lambda, h)=0$. Podemos supor sem perda de generalidade que $h=i_{\Omega}$ como já foi justificado na observação (3.1).

Calculando então a diferencial de $F$ no ponto crítico $\left(u, \lambda, i_{\Omega}\right)$ através do teorema (1.8) obtemos pela hipótese de absurdo que

$$
D F\left(u, \lambda, i_{\Omega}\right)(\dot{u}, \dot{\lambda}, \dot{h})=A_{\Omega}(\lambda) \dot{u}+\dot{\lambda} A_{\Omega}^{\prime}(\lambda) u+\left[\dot{h} \cdot \nabla, A_{\Omega}(\lambda)\right] u
$$




$$
=A_{\Omega}(\lambda)(\dot{u}-\dot{h} \cdot \nabla u)+\dot{\lambda} A_{\Omega}^{\prime}(\lambda) u
$$

não é sobrejetora.

A imagem de $D F\left(u, \lambda, i_{\Omega}\right)$ em $\left\{\dot{u} \in H^{2} \cap H_{0}^{1}(\Omega), \dot{\lambda}=0, \dot{h}=0\right\}$ coincide com a imagem do operador Fredholm $A_{\Omega}(\lambda)$ em $H^{2} \cap H_{0}^{1}(\Omega)$ que é fechada e possui codimensão finita. Agora, como tal conjunto está contido na imagem de $D F\left(u, \lambda, i_{\Omega}\right)$, podemos concluir que a imagem de $D F\left(u, \lambda, i_{\Omega}\right)$ tem codimensão finita e portanto é fechada. Como este operador é não sobrejetor, podemos afirmar também que a codimensão de sua imagem é positiva. Assim sendo, como $L^{2}(\Omega)$ é um espaço de Hilbert e a imagem de $D F\left(u, \lambda, i_{\Omega}\right)$ é fechada, existe $\psi \in L^{2}(\Omega)$, não nula, ortogonal a imagem de $D F\left(u, \lambda, i_{\Omega}\right)$.

Tomando $\dot{\lambda}=\dot{h}=0$ temos $\int_{\Omega} \psi A_{\Omega}(\lambda) \dot{u}=0 \forall \dot{u} \in L^{2}(\Omega)$, ou seja, $\psi$ é solução fraca de (1.10). Pelo fato de estarmos trabalhando com $A_{\Omega}(\lambda)$ em $H^{2} \cap H_{0}^{1}(\Omega)$, com $\Omega$ conexa limitada $\mathcal{C}^{3}$-regular satisfazendo as hipóteses citadas anteriormente, podemos afirmar que $\psi \in H^{2} \cap H_{0}^{1}(\Omega)$ e é solução forte de

$$
A_{\Omega}^{*}(\lambda) \psi=0 \text { em } \Omega, \psi=0 \text { em } \partial \Omega .
$$

Tal afirmação segue do teorema $\left(8.2^{\prime}\right)$ de [2]. Observe ainda que $u, \psi \in \mathcal{C}^{2}(\Omega) \cap H^{3}(\Omega)$, de fato, sendo $\Omega \in \mathcal{C}^{3}$, segue do teorema (15.2) de [6] que $u, \psi \in H^{3}(\Omega)$ e do seu corolário que $u, \psi \in \mathcal{C}^{2}(\Omega)$.

Então, $\forall \dot{h} \in \mathcal{C}^{3}\left(\Omega, R^{n}\right)$ temos

$$
\begin{aligned}
0 & =\int_{\Omega} \psi A_{\Omega}(\lambda) \dot{h} \cdot \nabla u \\
& =\int_{\Omega}\left\{\psi A_{\Omega}(\lambda) \dot{h} \cdot \nabla u-A^{*}(\lambda) \psi \dot{h} \cdot \nabla u\right\} \\
& =-\int_{\partial \Omega} \dot{h} \cdot \nabla u \sum_{i, j=1}^{n} \psi_{x_{i}} a_{i j}(x) N_{j} \\
& =-\int_{\partial \Omega} \frac{\partial u}{\partial N} \dot{h} \cdot N \sum_{j=1}^{n} N_{j} \nabla \psi \cdot\left(a_{1 j}(x), \ldots, a_{n j}(x)\right) \\
& =-\int_{\partial \Omega} \frac{\partial \psi}{\partial N} \frac{\partial u}{\partial N} \dot{h} \cdot N\left(\sum_{i, j=1}^{n} a_{i j}(x) N_{i} N_{j}\right) \forall \dot{h} \in \mathcal{C}^{3}\left(\Omega, \mathbb{R}^{n}\right) .
\end{aligned}
$$

Portanto $\frac{\partial \psi}{\partial N} \frac{\partial u_{0}}{\partial N} \equiv 0$ em $\partial \Omega$ já que $\left(\sum_{i, j=1}^{n} a_{i j}(x) N_{i} N_{j}\right) \geq c_{0}|N|^{2} \geq c_{0}$.

Logo nossa hipótese de absurdo implica na existência de $\psi \in L^{2}(\Omega)$, não nula, solução de (3.12) tal que $\frac{\partial \psi}{\partial N} \frac{\partial u_{0}}{\partial N} \equiv 0$ em $\partial \Omega$. Mas isto, como na primeira aplicação, viola a unicidade do problema de Cauchy para equações elípticas de segunda ordem (1.3), de onde podemos 
concluir que zero é valor regular de $F$. Portanto, genericamente em $\Omega$, todos os autovalores reais de (3.11) são simples.

\subsection{Simplicidade Genérica das soluções de $\Delta u+f(x, u, \nabla u)=0$}

Seja $Q$ um conjunto aberto em $\mathbb{R}^{n} \times \mathbb{R} \times \mathbb{R}^{n}$ e seja $f: Q \rightarrow \mathbb{R}$ uma função $\mathcal{C}^{2}$ limitada.

Provaremos que, genericamente no conjunto das regiões $\Omega \subset \mathbb{R}^{n}$ abertas, conexas, $\mathcal{C}^{3}$ regulares, limitadas, todas as soluções $u$ de

$$
\Delta u+f(x, u, \nabla u)=0 \text { em } \Omega, u=0 \text { sobre } \partial \Omega
$$

são simples, isto é, a linearização

$$
L_{u}: \dot{u} \longrightarrow \Delta \dot{u}+\frac{\partial f}{\partial p}(\cdot, u, \nabla u) \cdot \nabla \dot{u}+\frac{\partial f}{\partial u}(\cdot, u, \nabla u) \dot{u}
$$

é um isomorfismo. É importante observar que parte da definição de solução é que se $u$ de classe $\mathcal{C}^{1}$ é solução de (3.13), $(x, u, \nabla u) \in Q \forall x \in \bar{\Omega}$.

Segue diretamente do teorema da função inversa em espaços de Banach que, se todas as soluções de (3.13) são simples, tais soluções formam um conjunto discreto. Além disso, sendo $f$ limitada, afirmamos que o conjunto das soluções de (3.13) é finito. De fato, se $\Delta u+$ $f(x, u, \nabla u)=0,\|\Delta u\|_{L^{p}(\Omega)}=\|f(x, u, \nabla u)\|_{L^{p}(\Omega)} \leq M$ para algum $M>0$. Agora sendo $\Delta$ : $W^{2, p} \cap W_{0}^{1, p}(\Omega) \longrightarrow L^{p}(\Omega)$ um isomorfismo temos que $M \geq\|\Delta u\|_{L^{p}(\Omega)} \geq m\|u\|_{W^{2, p} \cap W_{0}^{1, p}(\Omega)}$

para algum $m>0$, o que nos dá $\|u\|_{W^{2, p} \cap W_{0}^{1, p}(\Omega)} \leq \frac{M}{m}$. Como $W^{2, p} \cap W_{0}^{1, p}(\Omega)$ está imerso em $L^{p}(\Omega)$ por imersão compacta e todas as soluções são discretas, temos que o número de soluções deve ser finito. Desta forma, se $f$ é limitada, a equação $\Delta u+f(x, u, \nabla u)=0 \mathrm{em}$ $\Omega$ com $u=0$ em $\partial \Omega$ tem somente um número finito de soluções.

Proposição 3.6 Se para algum $h \in \operatorname{Diff}^{3}(\Omega)$ a aplicação

$$
\begin{aligned}
F_{h}: W^{2, p} \cap W_{0}^{1, p}(\Omega) & \longrightarrow L^{p}(\Omega) \\
u & \longrightarrow h^{*}(\Delta+f) h^{*-1} u
\end{aligned}
$$

tem zero com valor regular, então todas as soluções de (3.13) em $h(\Omega)$ são simples.

Prova. 0 valor regular de $F_{h} \Longrightarrow h^{*} L_{u} h^{*-1}$ é sobrejetora para $u \in F_{h}{ }^{-1}(0)$. Agora o índice de Fredholm de tal aplicação é zero, logo tal aplicação é bijetora e então pelo teorema do Gráfico Fechado é um isomorfismo. 
Proposição $3.7 u \in W^{2, p} \cap W_{0}^{1, p}(\Omega)$ é solução simples de

$$
h^{*}(\Delta+f) h^{*-1} u=0 \text { em } \Omega, u=0 \text { sobre } \partial \Omega
$$

se somente se $v=h^{*-1} u$ é solução simples de (3.13) em $h(\Omega)$.

Prova. Seja $u \in W^{2, p} \cap W_{0}^{1, p}(\Omega)$, como $h^{*}$ é isomorfismo temos que

$$
h^{*}(\Delta+f) h^{*-1} u=0 \Longleftrightarrow(\Delta+f) h^{*-1} u=0,
$$

ou seja, $u$ é solução de (3.14) se somente se $h^{*-1} u$ é solução de (3.13) em $h(\Omega)$. Novamente usando o fato de que $h^{*}$ é isomorfismo temos que $h^{*} L_{u} h^{*-1}$ é isomorfismo em $W^{2, p} \cap W_{0}^{1, p}(\Omega)$ se somente se $L_{u}$ é isomorfismo em $W^{2, p} \cap W_{0}^{1, p}(h(\Omega))$.

A proposição (3.7) nos diz que estudar a simplicidade genérica das soluções de (3.13) é equivalente a estudar a simplicidade genérica das soluções de (3.14), portanto, de acordo com a proposição (3.6), obteremos a simplicidade genérica de todas as soluções de (3.13) se mostrarmos que para a maioria das $h \in \operatorname{Diff}^{3}(\Omega)$ a aplicação $F_{h}$ tem zero como valor regular.

Seja $E$ o seguinte subconjunto de $\mathcal{C}^{3}\left(\Omega, \mathbb{R}^{n}\right)$

$$
\begin{aligned}
E & =E_{1} \cup E_{2} \text { onde } \\
E_{1} & =\left\{h \in \mathcal{C}^{3}\left(\Omega, \mathbb{R}^{n}\right) ; f(x, 0,0) \equiv 0 \text { numa vizinhança de } \partial h(\Omega)\right\} \mathrm{e} \\
E_{2} & =\{f(x, 0,0) \neq 0 \text { em algum ponto de } \partial h(\Omega)\} .
\end{aligned}
$$

$E$ é aberto, de fato, dado $h_{0} \in E \operatorname{com} f(x, 0,0) \equiv 0$ numa vizinhança de $\partial h_{0}(\Omega)$ existe uma vizinhança $V_{h_{0}}$ de $h_{0}$ em $\mathcal{C}^{3}\left(\Omega, \mathbb{R}^{n}\right)$ tal que $f(x, 0,0) \equiv 0$ em $\partial h(\Omega) \forall h \in V_{h_{0}}$; se $f(x, 0,0) \neq 0$ em algum ponto de $\partial h_{0}(\Omega), f(x, 0,0) \neq 0$ numa vizinhança de $\partial h_{0}(\Omega)$ o que implica na existência de uma vizinhança $\tilde{V}_{h_{0}}$ de $h_{0}$ tal que $f(x, 0,0) \neq 0$ em algum ponto de $\partial h_{0}(\Omega)$ para todo $h \in \tilde{V}_{h_{0}}$. Portanto $E$ é aberto. $E$ também é denso. De fato, qualquer perturbação da região $h(\Omega)$ na direção da normal $N_{h}$ pertence a $E$. Logo podemos afirmar que $E$ é aberto e denso em $\mathcal{C}^{3}\left(\Omega, \mathbb{R}^{n}\right)$.

$\tilde{D}=E \cap \operatorname{Diff}^{3}(\Omega)$ é um subconjunto aberto e denso de $\operatorname{Diff}^{3}(\Omega)$. Observe que

$$
\begin{gathered}
\tilde{D}=\tilde{D_{1}} \cup \tilde{D_{2}} \text { onde } \\
\tilde{D_{1}}=\left\{E_{1} \cap \operatorname{Diff}^{3}(\Omega)\right\} \text { e } \tilde{D}_{2}=\left\{E_{2} \cap \operatorname{Diff}^{3}(\Omega)\right\}
\end{gathered}
$$

que são abertos disjuntos de $\operatorname{Diff}^{3}(\Omega)$.

Para todo $h \in \tilde{D}$ temos que $h \in \tilde{D}_{i}$ para $i=1$ ou $i=2$. Seja $V_{h}$ uma vizinhança de $h \in \tilde{D}_{i}$ contida em $\tilde{D}_{i}$ de maneira que se $\tilde{h} \in V_{h}, \partial \tilde{h}(\Omega)$ está contida numa vizinhança 
tubular de $\partial h(\Omega)$. O conjunto $\mathcal{C}$ de tais vizinhanças forma uma cobertura de abertos para $\tilde{D}$, portanto, sendo $\operatorname{Diff}^{3}(\Omega)$ Lindelof, $\tilde{D}$ é Lindelof e então podemos tomar uma subcobertura enumerável $\left\{V_{h_{1}}, \ldots, V_{h_{k}}, \ldots\right\}$ de $\tilde{D}$ onde cada $V_{h_{k}} \in \mathcal{C}$.

Provaremos que para cada $k$ e para todo $h \in V_{h_{k}}$, exceto um subconjunto $H_{k}$ magro de $\tilde{D}$ contido em $V_{h_{k}}$, todas as soluções de (3.13) em $h(\Omega)$ são simples. Mais precisamente, provaremos que para todo $h \in \cup_{k \geq 1}\left\{V_{h_{k}}-H_{k}\right\}$ todas as soluções de (3.13) em $h(\Omega)$ são simples. Observe que $\cup_{k \geq 1}\left\{V_{h_{k}}-H_{k}\right\}$ é um subconjunto denso de $\tilde{D}$, daí como $\tilde{D}$ é um subconjunto aberto e denso de $\operatorname{Diff}^{3}(\Omega)$ teremos que todas as soluções de (3.13) são genericamente simples em $\operatorname{Diff}^{3}(\Omega)$ como queremos mostrar.

Para isso considere a aplicação diferenciável

$$
\begin{aligned}
F: W^{2, p} \cap W_{0}^{1, p}(\Omega) \times V_{h_{k}} & \longrightarrow L^{p}(\Omega) \\
(u, h) & \longrightarrow h^{*}(\Delta+f) h^{*-1} u
\end{aligned}
$$

para algum $p$ com $n<p<\infty$ de tal maneira que $W^{2, p} \subset \mathcal{C}^{1}$ e algum $k \in \mathbb{N}$. Através do teorema da Transversalidade aplicado a $F$ mostraremos que existe $H_{k}$ magro em $V_{h_{k}}$, portanto magro em $\tilde{D}$, tal que em $\left\{V_{h_{k}}-H_{k}\right\}$ todas as soluções de (3.13) são simples, de onde segue o resultado desejado.

As hipóteses (1) e (3) do teorema para a aplicação $F$ são satisfeitas diretamente bastando apenas provar a sobrejetividade da diferencial de $F$.

Suponha por absurdo que exista $\left(u, i_{\Omega}\right)$ ponto crítico de $F \operatorname{com} F\left(u, i_{\Omega}\right)=0$, ou seja, a diferencial de $F$

$$
\begin{aligned}
D F\left(u, i_{\Omega}\right): W^{2, p} \cap W_{0}^{1, p}(\Omega) \times \mathcal{C}^{3}\left(\Omega, \mathbb{R}^{n}\right) & \longrightarrow L^{p}(\Omega) \\
(\dot{u}, \dot{h}) & \longrightarrow L_{u}(\dot{u}-\dot{h} \cdot \nabla u)
\end{aligned}
$$

não é sobrejetora. Como $D F\left(u, i_{\Omega}\right)$ é Fredholm não sobrejetor existe $\psi$ não nulo em $L^{q}(\Omega)$, $p^{-1}+q^{-1}=1$, ortogonal a imagem de $D F\left(u, i_{\Omega}\right)$ tal que

$$
\int_{\Omega} \psi L_{u}(\dot{u}-\dot{h} \cdot \nabla u)=0 \forall \dot{u} \in W^{2, p} \cap W_{0}^{1, p}(\Omega) \text { e } \forall \dot{h} \in \mathrm{C}^{3}\left(\Omega, \mathbb{R}^{n}\right)
$$

Pelo teorema (15.2) de [6] $u \in W^{3, p}(\Omega)$ já que $\Omega \in \mathrm{C}^{3}$. Daí pelo teorema (11.1) de [9] temos que $u \in \mathcal{C}^{2}(\Omega)$. Como $u$ é solução de (3.13) e $f \in \mathcal{C}^{2}$ temos pelo teorema (2.28) de [8] que $u \in \mathrm{C}^{2, \alpha}$ para todo $\alpha<1$, logo os coeficientes de $L_{u}$ são ao menos de classe $\mathrm{C}^{1}$.

Fixando $\dot{h}=0$ temos que $\int_{\Omega} \psi L_{u} \dot{u}=0 \quad \forall \dot{u} \in W^{2, p} \cap W_{0}^{1, p}(\Omega)$. Daí pelo teorema $\left(8.2^{\prime}\right)$ de [2] $\psi \in W^{2, p} \cap W_{0}^{1, p}(\Omega)$ e é solução de $L_{u}^{*} \psi=0$ em $\Omega$ com $\psi=0$ em $\partial \Omega$. Observe que $\psi$ como $u$ é de classe $\mathrm{C}^{2, \alpha}$. 
Tomando $\dot{u}=0$ temos que

$$
\begin{aligned}
0 & =\int_{\Omega} \psi L_{u}(\dot{h} \cdot \nabla u) \\
& =\int_{\Omega}\left\{\psi L_{u}(\dot{h} \cdot \nabla u)-(\dot{h} \cdot \nabla u) L_{u}^{*} \psi\right\} \\
& =\int_{\partial \Omega}\left\{\psi \frac{\partial(\dot{h} \cdot \nabla u)}{\partial N}-\frac{\partial \psi}{\partial N}(\dot{h} \cdot \nabla u)-\psi(\dot{h} \cdot \nabla u) \frac{\partial f}{\partial p} \cdot N\right\} \\
& =-\int_{\partial \Omega} \dot{h} \cdot N \frac{\partial \psi}{\partial N} \frac{\partial u}{\partial N} \forall \dot{h} \in \mathcal{C}^{3}\left(\Omega, \mathbb{R}^{n}\right)
\end{aligned}
$$

de onde obtemos que $\frac{\partial \psi}{\partial N} \frac{\partial u}{\partial N}=0$ em $\partial \Omega$. Agora

$$
\begin{gathered}
L_{u}^{*} \psi=0 \Longleftrightarrow \Delta \psi-\frac{\partial f}{\partial p}(\cdot, u, \nabla u) \cdot \nabla \psi+\left(\frac{\partial f}{\partial u}(\cdot, u, \nabla u)-\operatorname{div}\left(\frac{\partial f}{\partial p}(\cdot, u, \nabla u)\right)\right) \psi=0 \\
\Longrightarrow|\Delta \psi(x)| \leq\left|\frac{\partial f}{\partial p}(x, u, \nabla u) \cdot \nabla \psi(x)\right|+\left|\sum_{i=1}^{n} \frac{\partial^{2} f}{\partial x_{i} \partial p_{i}}(x, u, \nabla u) \psi(x)\right|+\left|\frac{\partial f}{\partial u}(x, u, \nabla u) \psi(x)\right|
\end{gathered}
$$

para todo $x \in \Omega$. Portanto sendo $\Omega$ limitado, $f \in \mathcal{C}^{2}$ e $u$ solução de (3.13) ao menos $\mathcal{C}^{1}$ temos que

$$
|\Delta \psi(x)| \leq C(|\nabla \psi(x)|+|\psi(x)|), \forall x \in \Omega
$$

onde

$$
C=\max _{x \in \bar{\Omega}}\left\{\left|\frac{\partial f}{\partial p}(x, u, \nabla u)\right|,\left|\sum_{i=1}^{n} \frac{\partial^{2} f}{\partial x_{i} \partial p_{i}}(x, u, \nabla u)\right|,\left|\frac{\partial f}{\partial u}(x, u, \nabla u)\right|\right\} .
$$

Logo, como $\psi \in W^{2, p} \cap W_{0}^{1, p}(\Omega)-\{0\}$ e satisfaz a desigualdade anterior, temos pelo teorema de Cauchy (1.3) que $\frac{\partial \psi}{\partial N} \neq 0$ em quase todo ponto de $\partial \Omega$ implicando que $\frac{\partial u}{\partial N}=0$ em $\partial \Omega$. Portanto a hipótese de contradição implica que a solução $u$ de (3.13) satisfaz ainda $\frac{\partial u}{\partial N}=0$ em $\partial \Omega$.

Como por hipótese $\Omega \in \tilde{D}$, temos que $i_{\Omega} \in \tilde{D}_{i}$ para $i=1$ ou $i=2$. Suponha que $i_{\Omega} \in \tilde{D_{1}}$. Então existe uma vizinhança $V$ de $\partial \Omega$ tal que $f(x, 0,0)=0$ para todo $x \in V$. Dado $x \in V$

$$
\begin{aligned}
|\Delta u(x)| & =|f(x, u(x), \nabla u(x))| \\
& =|f(x, u(x), \nabla u(x))-f(x, 0,0)| \\
& \leq K(|u(x)|+|\nabla u(x)|) \forall x \in V
\end{aligned}
$$

para algum $K>0$. Logo pelo teorema de unicidade do problema de Cauchy, como $u=$ $\frac{\partial u}{\partial N}=0$ em $\partial \Omega, u \equiv 0$ em $V$. Assim nós obtemos uma solução especial $u=u_{0}$ que se anula numa vizinhança $V$ de $\partial \Omega$, ou seja, obtemos uma solução $u_{0}$ de (3.13) que satisfaz (3.13) para todo $h \in V_{h_{k}}$ ( $u_{0}$ não depende de $\Omega$ para pequenas pertubações). Observe que $u_{0}$ está 
definida em $\Omega \cup V$ pois se anula em $V$ e $\partial \Omega \subset V$. Observe também que $u_{0}$ é única em $\Omega \cup V$ pois é única em $\Omega$. De fato, sejam $u_{0}^{1}$ e $u_{0}^{2}$ satisfazendo (3.13) em $\Omega$ e $\frac{\partial u_{0}^{i}}{\partial N}=0$ em $\partial \Omega i=1,2 . v=u_{0}^{1}-u_{0}^{2}$ satisfaz

$$
\Delta v+f\left(x, u_{0}^{1}, \nabla u_{0}^{1}\right)-f\left(x, u_{0}^{2}, \nabla u_{0}^{2}\right)=0 \text { em } \Omega, v=0 \text { e } \frac{\partial v}{\partial N}=0 \text { sobre } \partial \Omega .
$$

Para todo $x \in \Omega$ temos que

$$
\begin{aligned}
|\Delta v(x)| & =\left|f\left(x, u_{0}^{1}(x), \nabla u_{0}^{1}(x)\right)-f\left(x, u_{0}^{2}(x), \nabla u_{0}^{2}(x)\right)\right| \\
& \leq M(|v(x)|+|\nabla v(x)|)
\end{aligned}
$$

para algum $M>0$. Então segue pelo teorema de Cauchy (1.3) para operadores elípticos de segunda ordem que $v \equiv 0$ em $\Omega$ o que nos dá $u_{0}^{1} \equiv u_{0}^{2}$.

Suponha então que desde o início do argumento de contradição $u \in W^{2, p} \cap W_{0}^{1, p}(\Omega)$ $\left\{u_{0}\right\}$. Repetindo todo o argumento temos que $u \equiv u_{0}$ pois a solução especial $u_{0}$ é única em $\Omega$ de onde obtemos contradição, o que nos permite concluir que $D F$ é sobrejetora.

Sendo assim temos que todas as soluções de (3.13) são genericamente simples em $V_{h_{k}}$ exceto talvez a solução $u_{0}$. Mas tal solução também é genericamente simples. De fato, pelo exemplo anterior $L_{u_{0}}$ é um isomorfismo em $V_{h_{k}}$ exceto um subconjunto magro $H$. Então, como todas as outras soluções de (3.13) são simples em $V_{h_{k}}$ exceto um subconjunto magro $\tilde{H}_{k}$, segue-se que todas as soluções de (3.13) são simples em $V_{h_{k}}$ exceto um subconjunto magro $H_{k}=H \cup \tilde{H}_{k}$, de onde segue o resultado.

Suponha agora que $i_{\Omega} \in \tilde{D_{2}}$. Então $f(x, 0,0) \neq 0$ em algum ponto de $\partial \Omega$. Defina

$$
\begin{aligned}
G: W_{0}^{2, p}(\Omega) \times V_{h_{k}} & \longrightarrow L^{p}(\Omega) \\
(u, h) & \longrightarrow h^{*}(\Delta+f) h^{*-1} u .
\end{aligned}
$$

Note que $G$ é de classe $\mathcal{C}^{1}$ e que $u \in W^{2, p} \cap W_{0}^{1, p}(\Omega)$ está em $W_{0}^{2, p}(\Omega)$ se somente se $u=\frac{\partial u}{\partial N}=0$ em $\partial \Omega$.

Suponha que para todo $u \in W_{0}^{2, p}(\Omega)$ e para todo $h \in V_{h_{k}}$, exceto um subconjunto fechado e magro $O, G(u, h) \neq 0$. Sendo tal afirmação verdadeira podemos restringir $F$ ao subconjunto $\left\{V_{h_{k}}-O\right\}$ aberto e denso em $V_{h_{k}}$ e concluir que a solução $u$ de (3.13) satisfazendo $\frac{\partial u}{\partial N}=0$ em $\partial \Omega$ não poderá existir de onde obtemos uma contradição para nossa hipótese de absurdo implicando que $D F$ é sobrejetora e enfim segue-se o resultado desejado.

A demonstração de que $G(u, h) \neq 0$ genericamente em $V_{h_{k}}$ para todo $u \in W_{0}^{2, p}(\Omega)$ será feita usando a hipótese $(2 \beta)$ do teorema (2.6) no caso $n \geq 2$. O caso $n=1$ será estudado separadamente. 
Para usarmos o teorema verificaremos inicialmente a hipótese $(2 \beta)$ supondo $G\left(u, i_{\Omega}\right)=$ $0, n \geq 2$. Observe que $\frac{\partial G}{\partial u}\left(u, i_{\Omega}\right)=\left.L_{u}\right|_{W_{0}^{2, p}(\Omega)}$ é injetivo pelo teorema de unicidade de Cauchy para operadores elípticos.

Suponha

$$
\begin{aligned}
& m=\operatorname{dim}\left\{\operatorname{Im}\left(D G\left(u, i_{\Omega}\right)\right) / \operatorname{Im}\left(\frac{\partial G}{\partial u}\left(u, i_{\Omega}\right)\right)\right\}<\infty . \\
& D G\left(u, i_{\Omega}\right): W_{0}^{2, p}(\Omega) \times \mathcal{C}^{3}\left(\Omega, \mathbb{R}^{n}\right) \longrightarrow L^{p}(\Omega) \\
&(\dot{u}, \dot{h}) \longrightarrow L_{u}(\dot{u}-\dot{h} \cdot \nabla u)
\end{aligned}
$$

então existem $\left\{f_{1}, \ldots, f_{m}\right\}$ em $L^{p}(\Omega) \operatorname{com} f_{i}=L_{u}\left(\dot{u}_{i}-\dot{h}_{i} \cdot \nabla u\right) \dot{u}_{i} \in W_{0}^{2, p}(\Omega)$ e $\dot{h}_{i} \in \mathcal{C}^{3}\left(\Omega, \mathbb{R}^{n}\right)$ tal que para todo $(\dot{u}, \dot{h}) \in W_{0}^{2, p}(\Omega) \times \mathcal{C}^{3}\left(\Omega, \mathbb{R}^{n}\right)$ existem $\dot{v} \in W_{0}^{2, p}(\Omega)$ e únicos $c_{1}, \ldots, c_{m} \in \mathbb{R}$ tal que

$$
\sum_{i=1}^{m} c_{i} f_{i}+L_{u} \dot{v}=L_{u}(\dot{u}-\dot{h} \cdot \nabla u)
$$

de onde obtemos que

$$
\sum_{i=1}^{m} c_{i} L_{u}\left(\dot{u}_{i}-\dot{h}_{i} \cdot \nabla u\right)+L_{u} \dot{v}-L_{u}(\dot{u}-\dot{h} \cdot \nabla u)=0
$$

ou seja

$$
L\left(\dot{u}-\dot{v}-\sum_{i=1}^{m} c_{i} \dot{u}_{i}-\left(\dot{h}-\sum_{i=1}^{m} c_{i} \dot{h}_{i}\right) \cdot \nabla u\right)=0 .
$$

Seja $\left\{\phi_{1}, \ldots, \phi_{k}\right\}$ base do núcleo do operador de Fredholm $\left.L_{u}\right|_{W^{2, p} \cap W_{0}^{1, p}(\Omega)}$. Assim para constantes $b_{1}, \ldots, b_{k}$ temos que

$$
\dot{u}-\dot{v}-\sum_{i=1}^{m} c_{i} \dot{u}_{i}-\left(\dot{h}-\sum_{i=1}^{m} c_{i} \dot{h}_{i}\right) \cdot \nabla u=\sum_{i=1}^{k} b_{i} \phi_{i} .
$$

Já sabemos que $u \in W^{3, p}(\Omega)$. Logo $u \in W^{3, p} \cap W_{0}^{2, p}(\Omega)$ o que implica $\frac{\partial u}{\partial x_{i}} \in W^{2, p} \cap$ $W_{0}^{1, p}(\Omega)$ para cada $i$.

Calculando a derivada normal de tal expressão em $\partial \Omega$ temos que

$$
-\left(\dot{h}-\sum_{i=1}^{m} \dot{h}_{i}\right) \cdot N \frac{\partial^{2} u}{\partial N^{2}}=\sum_{i=1}^{k} b_{i} \frac{\partial \phi_{i}}{\partial N}
$$

Pelo teorema (1.2), $\Delta_{\partial \Omega} u=\Delta u-\operatorname{div}(N) \frac{\partial u}{\partial N}-\frac{\partial^{2} u}{\partial N^{2}}$. Como $u \equiv 0$ em $\partial \Omega$, temos que $\Delta_{\partial \Omega} u=0$. Portanto temos que em $\partial \Omega \frac{\partial^{2} u}{\partial N^{2}}=\left.\Delta u\right|_{\partial \Omega}=-f(x, 0,0)$. Assim

$$
\dot{h} \cdot N f(x, 0,0)=\sum_{i=1}^{m} c_{i} \dot{h}_{i} \cdot N f(x, 0,0)+\sum_{i=1}^{k} b_{i} \frac{\partial \phi_{i}}{\partial N} \text { em } \partial \Omega .
$$


Então para todo $\dot{h} \in \mathcal{C}^{3}\left(\Omega, \mathbb{R}^{n}\right)$ existem constantes $c_{1}, \ldots, c_{m}, b_{1}, \ldots, b_{k}$ tais que a equação anterior se verifica em $\partial \Omega$, ou seja, o operador limitado

$$
\left.\dot{h} \longrightarrow \dot{h} \cdot N f(x, 0,0)\right|_{\partial \Omega}
$$

tem posto finito. Como $\left.f(x, 0,0)\right|_{\partial \Omega} \neq 0$ e $n \geq 2$, ou seja $\operatorname{dim} \partial \Omega>0$, isto não pode ocorrer, contradição.

Logo $\operatorname{dim}\left\{\operatorname{Im}\left(D G\left(u, i_{\Omega}\right)\right) / \operatorname{Im}\left(\frac{\partial G}{\partial u}\left(u, i_{\Omega}\right)\right)\right\}$ é infinita e a condição $(2 \beta)$ está verificada. Como a $\operatorname{codim} \frac{\partial G}{\partial u} \geq \operatorname{dim}\left\{\operatorname{Im}\left(D G\left(u, i_{\Omega}\right)\right) / \operatorname{Im}\left(\frac{\partial G}{\partial u}\left(u, i_{\Omega}\right)\right)\right\}$ e $\frac{\partial G}{\partial u}$ é injetivo, o $i n d\left(\frac{\partial G}{\partial u}\right)=$ $-\infty \forall(u, h) \in G^{-1}(0)$, satisfazendo também a condição (1) do teorema da Transversalidade.

Agora, sendo $f$ limitada a aplicação $(u, h) \longrightarrow h: G^{-1}(0) \longrightarrow V_{h_{k}}$ é própria, de fato, $\left(u_{n}, h_{n}\right) \in G^{-1}(0) \longrightarrow\left\|\Delta h_{n}^{*-1} u_{n}\right\|_{L^{p}(\Omega)} \leq M \forall n$. Sendo $\Delta$ isomorfismo temos que passando a subsequência $\left\|h_{n}^{*-1} u_{n}\right\|_{L^{p}\left(h_{n}(\Omega)\right)}$ converge. Como $h_{n}^{*-1}$ é uma sequência de isomorfismos convergindo para um isomorfismo $h$ existem $m_{n}, m_{h}>0$ tal que $\left\|h_{n}^{*-1} u_{n}\right\|_{L^{p}\left(h_{n}(\Omega)\right)} \geq$ $m_{n}\left\|u_{n}\right\|_{W_{0}^{2, p}(\Omega)} \geq m_{h}\left\|u_{n}\right\|_{W_{0}^{2, p}(\Omega)}$ para todo $n$ o que nos dá $\left\{u_{n}\right\}$ uniformemente limitada em $W_{0}^{2, p}(\Omega)$. Mas, $\left\{u_{n}\right\}$ de fato é uniformemente limitada em $W^{3, p} \cap W_{0}^{2, p}(\Omega)$ já que $\Omega$ é uma região $\mathcal{C}^{3}$-regular. Portanto como $W^{3, p}(\Omega)$ está imerso compactamente em $W_{0}^{2, p}(\Omega)$, existe subsequência de Cauchy convergente em $W_{0}^{2, p}(\Omega)$, ou seja, existe $u \in W_{0}^{2, p}(\Omega)$ tal que $u_{n} \longrightarrow u$ em $W_{0}^{2, p}(\Omega)$ e segue a afirmação.

Verificado (3) podemos concluir pelo teorema da Transversalidade que se $n \geq 2 \forall h \in$ $V_{h_{k}}$, exceto para um subconjunto magro e fechado, não existe solução para (3.13) em $h(\Omega)$ satisfazendo $\frac{\partial u}{\partial N}=0$ em $\partial \Omega$. Basta agora considerar o caso $n=1$ para concluirmos nosso resultado.

Suponhamos então $n=1$.

$$
u^{\prime \prime}+f\left(x, u, u^{\prime}\right)=0 \text { em } a<x<b, u(a)=u(b)=0 .
$$

Perturbar $\Omega=(a, b)$ significa apenas mudar $a$ e $b$. Mostraremos que para muitas escolhas de $a$ e $b$ não existe solução de (3.15) com $u^{\prime}(a)=u^{\prime}(b)=0$. Seja $U(x, a)$ solução de (3.15) $\operatorname{com} U_{x}=0$ em $x=a$. Assuma que para algum $b>a f(b, 0,0) \neq 0$ e $U(b, a)=U_{x}(b, a)=0$. Considere a equação de duas variáveis $U(x, \alpha)=0$. Já que $U_{x x}(b, a)=f(b, 0,0) \neq 0$ temos que $\nabla U(b, a) \neq 0$ e então pelo teorema da função implícita existe uma única aplicação $\beta:(a-\epsilon, a+\epsilon) \longrightarrow \mathbb{R}$ tal que $U_{x}(\beta(\alpha), \alpha)=0$ para todo $\alpha \in(a-\epsilon, a+\epsilon)$, logo não existe solução para (3.15) em $W_{0}^{2, p}(\alpha, \beta)$ para $(\alpha, \beta)$ perturbação de $(a, b)$, a menos que $\beta=\beta(\alpha)$ e $U(\beta(\alpha), \alpha)=0$. 


\subsection{Hiperbolicidade Genérica dos Equilíbrios de $u_{t}=\left(a u_{x}\right)_{x}+$} $f(u)$

Considere a equação parabólica não linear

$$
u_{t}=\left(a u_{x}\right)_{x}+f(u), 0<x<1
$$

com condições de Neumann para o contorno $u_{x}(0, t)=u_{x}(1, t)=0$ onde $f: \mathbb{R} \longrightarrow \mathbb{R}$ é $\mathcal{C}^{2}$ e $a:[0,1] \longrightarrow \mathbb{R}^{+}=(0, \infty)$ é uma função $\mathcal{C}^{2}$.

Uma função $u$ é equilíbrio de (3.16) se somente se

$$
\left(a u_{x}\right)_{x}+f(u)=0,0<x<1
$$

com $u_{x}(0, t)=u_{x}(1, t)=0$. Dizemos ainda que $u$ equilíbrio de (3.16) é hiperbólico se somente se o operador

$$
L_{u} \phi=\left(a \phi_{x}\right)_{x}+f^{\prime}(u) \phi
$$

$\operatorname{com} \phi_{x}(0)=\phi_{x}(1)=0$ é um isomorfismo.

Dado $f$ de classe $\mathcal{C}^{2}$ considere a aplicação diferenciável

$$
\begin{aligned}
\psi_{f}: H_{\mathcal{N}}^{2}(0,1) & \longrightarrow L^{2}(0,1) \\
u & \longrightarrow\left(a u_{x}\right)_{x}+f(u)
\end{aligned}
$$

onde $H_{\mathcal{N}}^{2}(0,1)=\left\{u \in H^{2}(0,1) ; u_{x}(0)=u_{x}(1)=0\right\}$. Observe que tal conjunto é um subespaço fechado de $H^{2}(0,1)$ já que $H^{2}(0,1) \subset \mathcal{C}^{1}(0,1)$. Logo $H_{\mathcal{N}}^{2}(0,1)$ pode ser considerado um espaço de Banach.

Proposição 3.8 Os equilíbrios de (3.16) são todos hiperbólicos se somente se 0 é valor regular de $\psi_{f}$.

Prova. 0 é valor regular de $\psi_{f}$ se somente se $\forall u \in \psi^{-1}(0)$ o operador

$$
L_{u}: H_{\mathcal{N}}^{2}(0,1) \longrightarrow L^{2}(0,1): \phi \longrightarrow\left(a \phi_{x}\right)_{x}+f^{\prime}(u) \phi
$$

é sobrejetor. $L_{u}$ é um operador Fredholm de índice zero já que $\left(a(\cdot)_{x}\right)_{x}$ é Fredholm de índice zero e $f^{\prime}(u)(\cdot)$ é um operador compacto em $H_{\mathcal{N}}^{2}(0,1)$. Portanto temos que $L_{u}$ é sobrejetor se somente se $L_{u}$ é um isomorfismo.

Nesta seção faremos uma aplicação diferente do teorema (2.6). Não perturbaremos o domínio, mas sim a função $f$ na equação (3.16). Mostraremos que se $a \in \mathcal{C}^{2}\left([0,1], \mathbb{R}^{+}\right)$é uma função monótona, então todos os equilíbrios de (3.16) são hiperbólicos em $f: \mathbb{R} \longrightarrow \mathbb{R}$ 
$\mathcal{C}^{2}$ na topologia de Whitney [12] exceto em um conjunto magro, ou seja, todos os equilíbrios de (3.16) são genericamente hiperbólicos em $f \mathcal{C}^{2}$ na topologia de Whitney.

Para obtermos a hiperbolicidade genérica de todos os equilíbrios de (3.16) em $f$ na topologia de Whitney, mostraremos primeiro a hiperbolicidade genérica de todos os equilíbrios de (3.16) em $f$ no espaço de Banach $\mathcal{C}^{2}(\mathbb{R}, \mathbb{R})$. Provada a afirmação em $\mathcal{C}^{2}(\mathbb{R}, \mathbb{R})$, obtemos com certa facilidade a afirmação para $f$ de classe $\mathcal{C}^{2}$ na topologia de Whitney.

Dito isto, provemos primeiro que se $a \in \mathcal{C}^{2}\left([0,1], \mathbb{R}^{+}\right)$é uma função monótona, então para todo $f \in \mathcal{C}^{2}(\mathbb{R}, \mathbb{R})$, exceto um subconjunto magro, todos os equilíbrios de (3.16) são hiperbólicos.

Para isto, vamos mostrar que, dado $N \in \mathbb{N}$, todos os equilíbrios $u$ da equação (3.16) com $\|u\|_{H_{\mathcal{N}}^{2}(0,1)} \leq N$ são hiperbólicos para toda $f \in \mathcal{C}^{2}(\mathbb{R}, \mathbb{R})$, exceto em um conjunto magro. Logo, fazendo interseção enumerável obtemos que todos os equilíbrios de (3.16) são hiperbólicos em $\mathcal{C}^{2}(\mathbb{R}, \mathbb{R})$, exceto em um conjunto magro.

Inicialmente, vamos provar que os equilíbrios constantes de (3.16) com módulo limitado por $N \in \mathbb{N}$ são todos hiperbólicos num subconjunto aberto e denso de $\mathcal{C}^{2}(\mathbb{R}, \mathbb{R})$. De fato, seja $u=u_{0} \in \mathbb{R}$ equilíbrio constante de (3.16) e $\lambda_{0}, \lambda_{1}, \ldots, \lambda_{n}, \ldots$ os autovalores do operador

$$
T: H_{\mathcal{N}}^{2}(0,1) \longrightarrow L^{2}(0,1): \phi \longrightarrow\left(a \phi_{x}\right)_{x} .
$$

Observemos que $u_{0}$ é solução de (3.16) se somente se $f\left(u_{0}\right)=0$ e, nesse caso, $L_{u_{0}}$ é não sobrejetor se somente se $f^{\prime}\left(u_{0}\right)=\lambda_{i}$ para algum $i$. Observe que tais autovalores são enumeráveis e não possuem ponto de acumulação já que $T$ é um operador limitado de resolvente compacto. Seja $K \in \mathbb{N}$ o número de autovalores de $T$ contido no intervalo $[-N, N]$ e defina a aplicação contínua

$$
S:[-N, N] \times \mathcal{C}^{2}(\mathbb{R}, \mathbb{R}) \longrightarrow \mathbb{R} \times \mathbb{R}:(x, f) \longrightarrow\left(f(x), f^{\prime}(x)\right) .
$$

$S^{-1}\left(0, \lambda_{i}\right)$ é fechado em $\mathbb{R} \times \mathcal{C}^{2}(\mathbb{R}, \mathbb{R})$, portanto possui complementar $S^{-1}\left(0, \lambda_{i}\right)^{c}$ aberto. Seja $O_{\lambda_{i}}$ a projeção de $S^{-1}\left(0, \lambda_{i}\right)^{c}$ em $\mathcal{C}^{2}(\mathbb{R}, \mathbb{R})$. $O_{\lambda_{i}}$ é aberto já que a projeção é uma aplicação aberta. Afirmamos que $O_{\lambda_{i}}$ é também denso em $\mathcal{C}^{2}(\mathbb{R}, \mathbb{R})$. Seja $f$ uma função de classe $\mathcal{C}^{2}$ e considere a aplicação diferenciável

$$
\Lambda: \mathbb{R}^{3} \longrightarrow \mathbb{R}^{2}:(x, \lambda, \mu) \longrightarrow\left(f(x)+\lambda x+\mu, f^{\prime}(x)+\lambda\right) .
$$

Seja $(x, \lambda, \mu) \in \Lambda^{-1}\left(0, \lambda_{i}\right)$.

$$
D \Lambda(x, \lambda, \mu)=\left(\begin{array}{lll}
f^{\prime}(x)+\lambda & x & 1 \\
f^{\prime \prime}(x) & 1 & 0
\end{array}\right)
$$

é sobrejetora, portanto pelo teorema da Transversalidade de Thom a aplicação

$$
\Psi_{\lambda, \mu}: \mathbb{R} \longrightarrow \mathbb{R}^{2}: x \longrightarrow \Lambda(x, \lambda, \mu)
$$


possui $\left(0, \lambda_{i}\right)$ como valor regular num conjunto residual de $(\lambda, \mu) \in \mathbb{R}^{2}$. Agora, $\left(0, \lambda_{i}\right)$ ser valor regular de $\Psi_{\lambda, \mu}$ implica que o ponto $\left(0, \lambda_{i}\right)$ não pertence a imagem de $\Psi_{\lambda, \mu}$. Então, se $\Lambda(x, \lambda, \mu)=\left(0, \lambda_{i}\right)$, existem $\tilde{\lambda}, \tilde{\mu} \in \mathbb{R}$ próximos a $\lambda, \mu$ respectivamente, de maneira que $\left(f(x)+\tilde{\lambda} x+\tilde{\mu}, f^{\prime}(x)+\tilde{\lambda}\right) \neq\left(0, \lambda_{i}\right)$ para todo $x \in[-N, N]$. Suponha então que $f \in O_{\lambda_{i}}{ }^{c}$, ou seja, existe $u_{0} \in[-N, N]$ tal que $\Lambda\left(u_{0}, 0,0\right)=\left(0, \lambda_{i}\right)$. Pelo fato observado acima existem $\lambda, \mu \in \mathbb{R}$ próximos de 0 tal que

$$
\left(f(x)+\lambda x+\mu, f^{\prime}(x)+\lambda\right) \neq\left(0, \lambda_{i}\right) \text { para todo } x \in[-N, N] .
$$

De fato, $g(x)=f(x)+\lambda x+\mu$ pertence a $O_{\lambda_{i}}$. Modificando-a fora de $[-N, N]$ podemos obter $\tilde{g} \in O_{\lambda_{i}}$ tal que $\tilde{g}$ está próxima de $f$ em $\mathcal{C}^{2}(\mathbb{R}, \mathbb{R})$. Portanto $O_{\lambda_{i}}$ é denso como queríamos provar. Então $\cup_{i=0}^{K} O_{\lambda_{i}}{ }^{c}$ é um conjunto magro e fechado de $\mathcal{C}^{2}(\mathbb{R}, \mathbb{R})$ e todos os equilíbrios constantes de (3.16) limitados por $N$ são hiperbólicos no subconjunto aberto e denso de $\mathcal{C}^{2}(\mathbb{R}, \mathbb{R})$

$$
Y_{N}=\mathcal{C}^{2}(\mathbb{R}, \mathbb{R})-\cup_{i=0}^{K} O_{\lambda_{i}}{ }^{c} .
$$

De fato, para toda função constante $u \leq N$ equilíbrio de (3.16) temos que $f(u)=0$, daí como $f \in Y_{N}, f^{\prime}(u) \neq \lambda_{i}$ para $0 \leq i \leq K$ implicando que $u$ é equilíbrio hiperbólico de (3.16).

Sendo assim, para mostrarmos que todos os equilíbrios de (3.16) são genericamente hiperbólicos, basta mostrar que todos os equilíbrios de (3.16) limitados por $N$ são hiperbólicos em $Y_{N}$, exceto um subconjunto magro $X_{N}$ de $Y_{N}$, pois assim, todos os equilíbrios de (3.16) são hiperbólicos em $\mathcal{C}^{2}(\mathbb{R}, \mathbb{R})$ exceto o subconjunto magro $\left\{\cup_{i=0}^{\infty} X_{i}\right\} \cup\left\{\cup_{i=0}^{\infty} O_{\lambda_{i}}{ }^{c}\right\}$ de $\mathcal{C}^{2}(\mathbb{R}, \mathbb{R})$.

Para este fim, considere então a aplicação diferenciável

$$
\begin{aligned}
F: B_{N} \times Y_{N} & \longrightarrow L^{2}(0,1) \\
(u, f) & \longrightarrow\left(a u_{x}\right)_{x}+f(u)
\end{aligned}
$$

onde

$$
B_{N}=\left\{u \in H_{N}^{2}(0,1) ;\|u\|_{H_{N}^{2}(0,1)} \leq N\right\},
$$

$Y_{N}$ é o subconjunto aberto e denso de $\mathcal{C}^{2}(\mathbb{R}, \mathbb{R})$ definido anteriormente e $a \in \mathcal{C}^{1}\left([0,1], \mathbb{R}^{+}\right)$ é uma função monótona. Usando o teorema da Transversalidade mostraremos que para todo $f$ em $Y_{N}$, exceto um conjunto magro, a aplicação $u \longrightarrow F(u, f)$ tem zero como valor regular. Então pela proposição (3.8) segue que todos os equilíbrios de (3.16) em $B_{N}$ são genericamente hiperbólicos em $Y_{N}$ de onde segue o resultado desejado.

As hipóteses (1) e (3) do teorema da transversalidade são imediatas, bastando apenas verificar a hipótese $2(\alpha)$. 
Suponha por absurdo que exista $(u, f) \in F^{-1}(0)$ tal que a diferencial

$$
\begin{aligned}
D F(u, f): H_{N}^{2}(0,1) \times \mathcal{C}^{2}(\mathbb{R}, \mathbb{R}) & \longrightarrow L^{2}(0,1) \\
(\dot{u}, \dot{f}) & \longrightarrow\left(a \dot{u}_{x}\right)_{x}+f^{\prime}(u) \dot{u}+\dot{f}(u)
\end{aligned}
$$

não é sobrejetora.

Observe que a função $u$ não pode ser constante, de fato, se $u$ fosse constante o conjunto $\left\{D F(u, f)(\dot{u}, 0) ; \dot{u} \in H_{\mathcal{N}}^{2}(0,1)\right\}$ seria igual a $L^{2}(0,1)$ já que $f \in Y_{N}$ implicando que o operador $D F(u, f)$ é sobrejetor.

Então $u$ é não constante e como sempre, existe $\phi \in L^{2}(0,1)$ não nula ortogonal à imagem do operador $D F(u, f)$, ou seja,

$$
\int_{0}^{1} \phi\left\{\left(a \dot{u}_{x}\right)_{x}+f^{\prime}(u) \dot{u}+\dot{f}(u)\right\}=0 \forall \dot{u} \in H_{\mathcal{N}}^{2}(0,1) \text { e } \forall \dot{f} \in \mathcal{C}^{2}(\mathbb{R}, \mathbb{R}) .
$$

Tomando $\dot{f}=0$ temos que $\int_{0}^{1} \phi\left\{\left(a \dot{u}_{x}\right)_{x}+f^{\prime}(u) \dot{u}\right\}=0 \forall \dot{u} \in H_{\mathcal{N}}^{2}(0,1)$ implicando que $\phi \in H_{\mathcal{N}}^{2}(0,1) \cap \mathcal{C}^{2}(0,1)$ é solução fraca, portanto solução forte de

$$
L_{u} \phi=0 \text { em } 0<x<1
$$

já que $a>0$. Fazendo agora $\dot{u}=0$ temos que $\int_{0}^{1} \dot{f}(u) \phi=0 \forall \dot{f} \in \mathcal{C}^{2}(\mathbb{R}, \mathbb{R})$.

Lema 3.9 Seja $u:[0,1] \longrightarrow \mathbb{R}$ solução não constante de (3.17). Se $\int_{0}^{1} \dot{f}(u) \phi d x=0 \forall \dot{f} \in$ $\mathcal{C}^{2}(\mathbb{R}, \mathbb{R})$ temos que

$$
2 \sum_{p \in I_{q} \cap(0,1)} \frac{\phi(p)}{\sqrt{\left|u^{\prime \prime}(p)\right|}}+\sum_{p \in I_{q} \cap\{0,1\}} \frac{\phi(p)}{\sqrt{\left|u^{\prime \prime}(p)\right|}}=0
$$

onde $I_{q}=\left\{p \in[0,1] ; u(p)=q\right.$ e $\left.u^{\prime}(p)=0\right\}$.

Prova. Observe que $u$, equilíbrio de (3.17) é uma função $\mathcal{C}^{3}(0,1)$, de fato,

$$
\left(a u_{x}\right)_{x}=-f(u) \in \mathcal{C}^{1}(0,1) \longrightarrow a u_{x} \in \mathcal{C}^{2}(0,1)
$$

com $a \in \mathcal{C}^{2}\left([0,1], \mathbb{R}^{+}\right)$. Observe agora que $I_{q}$ é um conjunto finito, já que $u$ é solução não constante de (3.17). De fato, se $I_{q}$ fosse ao menos enumerável, existiria $p \in I_{q}$ tal que $u^{\prime \prime}(p)=0$ implicando que $u_{x} \in \mathcal{C}^{2}(0,1)$ é solução da equação de segunda ordem

$$
(a v)_{x x}+f^{\prime}(u) v=0
$$

satisfazendo $u_{x}(p)=u_{x x}(p)=0$, e então, pelo teorema de unicidade de EDOs $u_{x} \equiv 0$ e $u$ é constante, absurdo. 
Suponha $p \in(0,1)$ um ponto crítico da função $u$. Numa vizinhança de $p$ podemos escrever $u$ como

$$
u(x)=u(p)+\frac{u^{\prime \prime}(p)}{2}(x-p)^{2}+O(x-p)^{3} .
$$

Sendo $u$ solução não constante de (3.17) temos que $u^{\prime \prime}(p) \neq 0$. Suponha $u^{\prime \prime}(p)<0$, (o caso positivo é análogo). A imagem de um pequeno intervalo $I=\left(p-\delta_{1}, p+\delta_{2}\right)$ ao redor de $p$ é então um intervalo da forma $(q-\epsilon, q]$, onde $q=u(p)$ e $\epsilon>0$. Então para $x \in I$ e $y \in[q-\epsilon, q]$ temos que

$$
\begin{aligned}
u(x)=y & \Longleftrightarrow y-q=\frac{u^{\prime \prime}(p)}{2}(x-p)^{2}+O\left(|(x-p)|^{3}\right) \\
& \Longleftrightarrow y-q=\frac{u^{\prime \prime}(p)}{2}(x-p)^{2}+O\left(\left|(x-p)^{2}\right|^{\frac{3}{2}}\right) \\
& \Longleftrightarrow(x-p)^{2}=\frac{2}{u^{\prime \prime}(p)}(y-q)+O\left(|y-q|^{\frac{3}{2}}\right)
\end{aligned}
$$

Observe que $u$ é um a um em cada um dos intervalos $\left[p-\delta_{1}, p\right]$ e $\left[p, p+\delta_{2}\right]$ sobre $[q-\epsilon, q]$, com

$$
\begin{aligned}
(x-p) & = \pm \sqrt{\frac{2(y-q)}{u^{\prime \prime}(p)}+O\left(|y-q|^{\frac{3}{2}}\right)} \\
& = \pm \sqrt{\frac{2(y-q)}{u^{\prime \prime}(p)}} \sqrt{1+O\left(|y-q|^{\frac{1}{2}}\right)} \\
& = \pm \sqrt{\frac{2(y-q)}{u^{\prime \prime}(p)}}\left(1+O\left(|y-q|^{\frac{1}{2}}\right)\right)
\end{aligned}
$$

ou seja,

$$
(x-p)= \pm \sqrt{\frac{2(y-q)}{u^{\prime \prime}(p)}}+O(|y-q|) .
$$

Seja $g_{\epsilon}: \mathbb{R} \longrightarrow \mathbb{R}$ a seguinte função:

$$
g_{\epsilon}= \begin{cases}\frac{1}{\sqrt{\epsilon}} & \text { se }|y-q| \leq \epsilon \\ 0 & \text { caso contrário. }\end{cases}
$$

Através de (3.19) podemos determinar aproximadamente o valor de $\delta_{1}$ :

$$
\delta_{1}=\sqrt{\frac{2 \epsilon}{\left|u^{\prime \prime}(p)\right|}}+O(\epsilon) .
$$

Portanto, a integral

$$
\int_{p-\delta_{1}}^{p} g_{\epsilon}(u(x)) \phi(x) d x=\int_{p-\delta_{1}}^{p} \frac{1}{\sqrt{\epsilon}} \phi(x) d x
$$




$$
\begin{aligned}
& =\frac{1}{\sqrt{\epsilon}} \phi(\bar{x}) \delta_{1} \\
& =\frac{1}{\sqrt{\epsilon}} \phi(\bar{x})\left(\sqrt{\frac{2 \epsilon}{\left|u^{\prime \prime}(p)\right|}}+O(\epsilon)\right) \\
& =\phi(\bar{x})\left(\sqrt{\frac{2}{\left|u^{\prime \prime}(p)\right|}}+O(\sqrt{\epsilon})\right) .
\end{aligned}
$$

para algum $\bar{x} \in\left(p-\delta_{1}, p\right)$. Fazendo $\epsilon \longrightarrow 0$ temos que a integral tende a

$$
\phi(p) \sqrt{\frac{2}{\left|u^{\prime \prime}(p)\right|}}
$$

De maneira análoga obtemos o mesmo valor para a integral $\int_{p}^{p+\delta_{2}} g_{\epsilon}(u(x)) \phi(x) d x$ quando $\epsilon \longrightarrow 0$. Então somando sobre todos os $p \in u^{-1}(q)$ temos que

$$
\int_{0}^{1} g_{\epsilon}(u(x)) \phi(x) d x \longrightarrow \sqrt{2}\left(2 \sum_{p \in I_{q} \cap(0,1)} \frac{\phi(p)}{\sqrt{\left|u^{\prime \prime}(p)\right|}}+\sum_{p \in I_{q} \cap\{0,1\}} \frac{\phi(p)}{\sqrt{\left|u^{\prime \prime}(p)\right|}}\right)
$$

quando $\epsilon \longrightarrow 0$, onde $I_{q}=\left\{p \in[0,1] ; u(p)=q\right.$ e $\left.u^{\prime}(p)=0\right\}$. Observe que qualquer função em $\mathcal{C}^{2}(\mathbb{R}, \mathbb{R})$ pode ser aproximada por funções do tipo $g_{\epsilon}$. Portanto, podemos afirmar que

$$
2 \sum_{p \in I_{q} \cap(0,1)} \frac{\phi(p)}{\sqrt{\left|u^{\prime \prime}(p)\right|}}+\sum_{p \in I_{q} \cap\{0,1\}} \frac{\phi(p)}{\sqrt{\left|u^{\prime \prime}(p)\right|}}=0
$$

já que por hipótese $\int_{0}^{1} \dot{f}(u) \phi d x=0 \forall \dot{f} \in \mathcal{C}^{2}(\mathbb{R}, \mathbb{R})$.

Observe que se $I_{u(0)}=\{0\}$ temos que $\phi(0)=0$. Como $\phi \in H_{\mathcal{N}}^{2}(0,1) \cap \mathcal{C}^{2}(0,1)$ e satisfaz (3.18), temos pelo teorema de unicidade de soluções de EDOs que $\phi \equiv 0$, contradizendo o fato de que $\phi$ é não nula. Portanto, $\phi(0) \neq 0$.

Dado $p \in I_{u(0)}$ temos que $a(p) u_{x x}(p)+f(u(p))=0$, o que nos dá $u_{x x}(p)=-\frac{f(u(0))}{a(p)}$. Portanto, já que $a>0$ temos que para todo $p \in I_{u(0)}, u_{x x}(p)$ possui o mesmo sinal. Então, se $u_{x x}(p)>0$ (o caso negativo é análogo) temos que $\forall p \in I_{u(0)}$

$$
2 \sum_{p \in I_{u(0)} \cap(0,1)} \frac{\phi(p)}{\sqrt{u^{\prime \prime}(p)}}+\sum_{p \in I_{u(0) \cap\{0,1\}}} \frac{\phi(p)}{\sqrt{u^{\prime \prime}(p)}}=0 .
$$

Defina a aplicação diferenciável

$$
\omega(x, v(x), w(x))=\frac{1}{2}\left(a(w(x))^{2}+\int_{0}^{x} a_{x}(w(s))^{2} d s\right)+G(v(x))
$$

onde $G$ é uma primitiva de $f$. Sendo $u$ solução de (3.17) temos que

$$
\begin{aligned}
\omega_{x}\left(x, u, u_{x}\right) & =\left(\frac{1}{2}\left(a u_{x}^{2}+\int_{0}^{x} a_{x} u_{x}^{2} d s\right)+G(u)\right)_{x} \\
& =u_{x}\left(a u_{x x}+a_{x} u_{x}+f(u)\right) \\
& =0 .
\end{aligned}
$$


Portanto, $\omega\left(x, u, u_{x}\right)$ é uma função constante e para $p \in I_{u(0)}$ temos que

$$
\begin{aligned}
0 & =\omega(p, u(0), 0)-\omega(0, u(0), 0) \\
& =\frac{1}{2} \int_{0}^{p} a_{x} u_{x}^{2} d s .
\end{aligned}
$$

Como $u$ não é constante em $[0,1]$, para todo $\epsilon>0$ a função $u_{x}$ é não nula em $(0, \epsilon)$. De fato, se isto não fosse verdade a solução constante $v=u(0)$ satisfaria a EDO (3.1.7) com valor inicial $v=u(0)$ e então pelo teorema de unicidade de EDOs $v=u$ mas por hipótese $u$ não é constante. Logo podemos concluir que $a_{x} \equiv 0$ em $[0, p]$, já que $a$ é uma função monótona, e daí temos que a equação (3.16) é uma equação com coeficiente constante em $[0, p]$ para todo $p \in I_{u(0)}$.

Seja $\tilde{p}=\max \left\{I_{u(0)}\right\}, \tilde{p} \in I_{u(0)}$, de fato, como foi visto na demostração do lema, o conjunto $I_{q}$ é finito para todo $q$ já que a função $u$ não é constante.

Então, até aqui, nossa hipótese de absurdo implica na existência de uma função $\phi$ solução clássica da equação (3.18) e que $a$ é constante em $[0, \tilde{p}] \subset[0,1]$.

Considere agora a equação

$$
a v_{x x}+f^{\prime}(u) v=0,0<x<\tilde{p} .
$$

Já que (3.16) é uma equação com coeficiente de difusão constante em $[0, \tilde{p}]$ temos que as funções $u_{x}$ e $\phi$ satisfazem (3.20) com $u_{x}(0)=0, u_{x x}(0) \neq 0, \phi(0) \neq 0$ e $\phi_{x}(0)=0$. O Wronskiano de (3.20) é constante para qualquer duas soluções dadas, portanto temos que para alguma constante $c$

$$
u_{x} \phi_{x}-u_{x x} \phi=c \forall x \in[0, \tilde{p}] .
$$

Dado $p \in I_{u(0)}$ temos que $p \in[0, \tilde{p}]$ e

$$
\phi(p)=-\frac{c}{u_{x x}(p)}=\frac{c a}{f(u(0))}
$$

que é constante em $I_{u(0)}$.

Então, pelo lema (3.9) temos que

$$
\frac{c a}{f(u(0))}\left(2 \sum_{p \in I_{u(0)} \cap(0,1)} \frac{1}{\sqrt{\left|u^{\prime \prime}(p)\right|}}+\sum_{p \in I_{u(0)} \cap\{0,1\}} \frac{1}{\sqrt{\left|u^{\prime \prime}(p)\right|}}\right)=0
$$

de onde podemos concluir que $\phi(p) \equiv 0 \forall p \in I_{u(0)}$ e portanto $\phi(0)=0$ contradizendo nossa hipótese de absurdo. Logo a hipótese $2(\alpha)$ do teorama da Transversalidade está verificada e segue afirmação de que todos os equilíbrios de (3.16) são hiperbólicos em $\mathcal{C}^{2}(\mathbb{R}, \mathbb{R})$, exceto em um conjunto magro.

Vamos agora mostrar o resultado principal desta seção. 
Teorema 3.10 Se $a \in \mathcal{C}^{2}\left([0,1], \mathbb{R}^{+}\right)$é uma função monótona, então todos os equilíbrios de

$$
u_{t}=\left(a u_{x}\right)_{x}+f(u), 0<x<1
$$

com condições de Neumann para o contorno $u_{x}(0, t)=u_{x}(1, t)=0$ são genericamente hiperbólicos em $f \mathcal{C}^{2}$ na topologia de Whitney.

Prova. Para provar o teorema, basta mọtrar que o conjunto $\mathcal{F}_{N}$ das $f$ na topologia de Whitney que possui todos os equilíbrios em $B_{N} \subset H_{\mathcal{N}}^{2}(0,1)$ hiperbólicos é aberto e denso na topologia de Whitney já que tal espaço topológico é de Baire.

Primeiro observe que $\mathcal{F}_{N}$ é denso. Seja $f$ uma função de classe $\mathcal{C}^{2}$. Como todos os equilíbrios de (3.16) pertencem a $B_{N}$, existe um intervalo $[a, b] \subset \mathbb{R}$ tal que a imagem de todos estes equilíbrios estão contidos em $[a, b]$. Tome $g \in \mathcal{C}^{2}(\mathbb{R}, \mathbb{R})$ tal que $g=f$ em $[a, b]$. Se $g$ não possui todos os seus equilíbrios hiperbólicos em $B_{N}$ existe $\bar{g} \in \mathcal{C}^{2}(\mathbb{R}, \mathbb{R})$ próxima a $g$ em $\mathcal{C}^{2}(\mathbb{R}, \mathbb{R})$ que possui todos os equilíbrios hiperbólicos pelo que foi demonstrado antes no exemplo. Seja $\tilde{f}=f-g+\bar{g}$. $\tilde{f}$ está próxima a $f$ na topologia de Whitney e todos os equilíbrios de (3.16) em $B_{N}$ para $\tilde{f}$ são hiperbólicos. Portanto $\mathcal{F}_{N}$ é denso na topologia de Whitney.

Provemos agora que $\mathcal{F}_{N}$ é aberto. Suponha por absurdo que exista uma sequência $\left\{f_{n}\right\}$ fora de $\mathcal{F}_{N}$ convergindo para $f \in \mathcal{F}_{N}$. Então existem sequências $\left\{u_{n}\right\} \subset B_{N}$ e $\left\{\phi_{n}\right\} \subset H_{\mathcal{N}}^{2}(0,1), \int_{0}^{1}\left(\phi_{n}\right)^{2}=1$ satisfazendo

$$
\left(a\left(u_{n}\right)_{x}\right)_{x}+f_{n}\left(u_{n}\right)=0 \text { e }\left(a\left(\phi_{n}\right)_{x}\right)_{x}+f_{n}^{\prime}\left(u_{n}\right) \phi_{n}=0 \forall n .
$$

Na demonstração do lema (3.9) vimos que $u_{n} \in \mathcal{C}^{3}(0,1)$. Portanto cada $u_{n}$ pertence a $H^{3}(0,1) \cap H_{\mathcal{N}}^{2}(0,1)$. Observe também que cada $u_{n}$ possui imagem em $[a, b]$ e portanto a sequência $\left\{u_{n}\right\}$ é uniformemente limitada em $H^{3}(0,1)$. Como $H^{3}(0,1)$ está imerso compactamente em $H^{2}(0,1)$, passando a subsequência, existe $u \in H_{\mathcal{N}}^{2}(0,1)$ tal que $u_{n} \longrightarrow u$. Por argumento análogo temos que cada $\phi_{n} \in H^{3}(0,1) \cap H_{\mathcal{N}}^{2}(0,1)$ e, passando a subsequência, existe $\phi \in H_{\mathcal{N}}^{2}(0,1)$ não nula já que $\int_{0}^{1}\left(\phi_{n}\right)^{2}=1$ tal que $\phi_{n} \longrightarrow \phi$. Sendo assim, quando $n \longrightarrow \infty$ temos que

$$
\left(a u_{x}\right)_{x}+f(u)=0 \text { e }\left(a \phi_{x}\right)_{x}+f^{\prime}(u) \phi=0 .
$$

Logo, nossa hipótese de absurdo produziu um equilíbrio $u \in B_{N}$ para $f$ que não é hiperbólico, absurdo. 


\section{Referências Bibliográficas}

[1] R. Abraham e J. Bobin, Transversal mappings and flows, W. A. Benjamin, 1967.

[2] S. Agmon, The $L_{p}$ approach to the dirichlet problem, Ann. Scuola Norm. Sup. Pisa, vol. 13, 405-448 (1959),.

[3] _ Unicité et convexité dans les problems differentielles, Univ. Montreal.

[4] E. Browder, Estimates and existence theorems for elliptic boundary value problems, Proc. Nat. Acad. Sci. USA, vol. 45, 385-372 (1959).

[5] M. Chicco, Solvability of the dirichlet problem in for a class of linear second order elliptic partial differential equations, Bollet. U. M. I., vol. 4, 374-387 (1971).

[6] S. Agmon; A. Douglis e N. Nirenberg, Estimates near the boundary for solutions of elliptic partial differential equations satisfyng general boundary conditions, Comm. Pure Appl. Math., vol. 12, 623-727 (1959).

[7] S. Chow e P. Brunovsky, Generic properties of stationary state solutions of reactiondiffusion equations, Journal Differential Equations, vol. 53, 1-23 (1984).

[8] G. B. Folland, Introduction to partial differential equations, Princeton University Press, 1995.

[9] A. Friedman, Partial differential equations, Holt Rinehart and Winston, 1969.

[10] D. B. Henry, Topics in nonlinear analysis, UnB, 1982.

[11] _ Perturbation of the boundary in boundary value problems of pdes, notas não publicadas, 1985.

[12] M. W. Hirsch, Differential topology, Springer Verlag, 1976. 
[13] L. Hormander, Linear partial differential operators, Springer Verlag, 1964.

[14] T. Kato, Perturbation theory for linear operators, Springer Verlag, 1980.

15] A. M. Micheletti, Perturbazione dello spettro dell'operatore de laplace in relazione ad una variazone del campo, Ann. Scuola Norm. Sup. Pisa, vol. 26, 151-169 (1972).

16] A. L. Pereira, Generic hiperbolicity for scalar parabolic equations, Proc. Royal Soc. Edinburgh, vol. 123A, 1031-1040 (1993).

.7] K. Uhlenbeck, Eigenfunctions of laplace operators, Bull. Am. Math. Soc., vol. 78,
1073-1076 (1972). 